July, 1997

\title{
SWEPP PAN Assay System Uncertainty Analysis: Passive Mode Measurements of Graphite Waste
}

Larry G. Blackwood

Yale D. Harker

Teresa R. Meachum

Woo Y. Yoon 


\title{
SWEPP PAN Assay System Uncertainty Analysis: Passive Mode Measurements of Graphite Waste
}

\author{
Larry G. Blackwood \\ Yale D. Harker \\ Teresa R. Meachum \\ Woo Y. Yoon
}

Published July 1997

\begin{abstract}
Idaho National Engineering and Environmental Laboratory
TRU Waste Programs

Lockheed Martin Idaho Technologies

Idaho Falls, Idaho 83415
\end{abstract}

\section{Prepared for the}

U. S. Department of Energy

Office of Environmental Restoration and Waste Management

Under DOE Idaho Operations Office

Contract DE-AC07-94ID13223

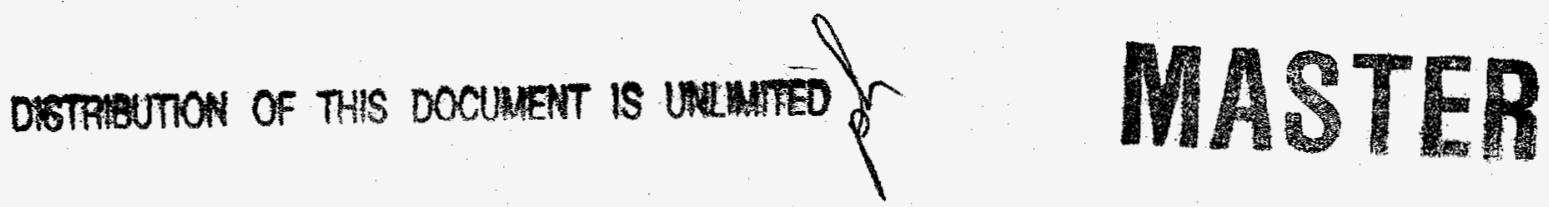




\section{DISCLAIMER}

This report was prepared as an account of work sponsored by an agency of the United States Government. Neither the United States Government nor any agency thereof, nor any of their employees, makes any warranty, express or implied, or assumes any legal liability or responsibility for the accuracy, completeness, or usefulness of any information, apparatus, product, or process disclosed, or represents that its use would not infringe privately owned rights. Reference herein to any specific commercial product, process, or service by trade name, trademark, manufacturer, or otherwise does not necessarily constitute or imply its endorsement, recommendation, or favoring by the United States Government or any agency thereof. The views and opinions of authors expressed herein do not necessarily state or reflect those of the United States Government or any agency thereof. 


\section{DISCLAIMER}

Portions of this document may be illegible electronic image products. Images are produced from the best available original document. 


\section{ABSTRACT}

The Idaho National Engineering and Environmental Laboratory is being used as a temporary storage facility for transuranic waste generated by the U. S. Nuclear Weapons program at the Rocky Flats Plant (RFP) in Golden, Colorado. Currently, there is a large effort in progress to prepare to ship this waste to the Waste Isolation Pilot Plant (WIPP) in Carlsbad, New Mexico. In order to meet the TRU Waste Characterization Quality Assurance Program Plan nondestructive assay compliance requirements and quality assurance objectives, it is necessary to determine the total uncertainty of the radioassay results produced by the Stored Waste Examination Pilot Plant (SWEPP) Passive Active Neutron (PAN) radioassay system. To this end a modified statistical sampling and verification approach has been developed to determine the total uncertainty of a PAN measurement. In this approach the total performance of the PAN nondestructive assay system is simulated using computer models of the assay system and the resultant output is compared with the known input to assess the total uncertainty. This paper is one of a series of reports quantifying the results of the uncertainty analysis of the PAN system measurements for specific waste types and measurement modes. In particular this report covers passive mode measurements of weapons grade plutonium-contaminated graphite molds contained in 208 liter drums (waste code 300 ).

The validity of the simulation approach is verified by comparing simulated output against results from measurements using known plutonium sources and a surrogate graphite waste form drum. For actual graphite waste form conditions, a set of 50 cases covering a statistical sampling of the conditions exhibited in graphite wastes was compiled using a Latin hypercube statistical sampling approach. The distributions from which Latin hypercube sample was drawn was derived from reviews of approximately 100 real-time radiography video tapes of RFP graphite waste drums, results from previous PAN measurements on graphite waste drums, and shipping data from RFP where the graphite waste was generated. The data in the 50 selected cases form the multi-parameter input to the simulation model. The reported plutonium masses from the simulation model are compared with corresponding input masses. From these comparisons, the bias and total uncertainty associated with PAN measurements on graphite waste drums is determined.

Note: This report replaces a previous report regarding the uncertainty analysis of graphite waste drum measurements (Harker et al., 1995). Since the publication of that report, there have been significant changes in the manner in which the PAN system operates (e.g., the addition of mass calculations based on shift register coincidence counts) as well as changes in the simulation routines. The description of the PAN system, the structure of the uncertainty analysis, and the resulting uncertainty estimates reported herein supersede the information found in the previous report. 


\section{SUMMARY}

This report covers a methodology for determining the total uncertainty of the PAN drum assay system and applies this methodology to the graphite content code 300 . In order to perform this evaluation, a complete assessment of the physical and radiological characteristics of graphite content code 300 waste drums was performed. A multivariate statistical model was developed which accounted for variations and correlations of parameters which could alter the response of the assay system. The primary parameters in this model are: mass of weapons grade PU, mass fraction of Pu-240, fill height of the drum, average matrix density, absorber index, background singles counts, background coincidence counts and fraction of total Pu contained in Pu chunks. Real time radiography tapes, data from past measurements using the PAN assay system and shipping records from Rocky Flats Plant were the sources of empirical data used in this assessment. From this study a set of 50 cases simulating the waste characteristics for graphite were prepared for analysis using a computer simulation of the assay system response and analysis routines.

The computer simulation of the PAN assay system response involved a neutron transport calculation using MCNP and a follow-up program which uses the MCNP results along with other parameters in the statistical model to determine the singles and coincidence count rate responses. These count rate data were then analyzed to yield Pu mass using exactly the same algorithms as contained in the PAN analysis program. The accuracy of the combined simulation routine was verified using data taken from measurements involving a surrogate graphite waste drum and disk Pu sources. From these comparisons small adjustment factors were derived to make the simulated Pu mass quantities agree on average with the Pu mass determined from the PAN measurement. These adjustment factors were included in the computer simulation routine in all analyses involving the simulated waste drum cases so that there was no bias in the simulation results relative to the expected PAN results.

Analysis of the results for the simulated waste drums indicates that, on average, there is no bias in the PAN system measurements of graphite waste. Approximate $95 \%$ confidence bounds on the PAN system bias for graphite drums are $(-2 \%, 2 \%)$. The limits on the bias easily meet Quality Assurance Program Plan requirements for radioassay systems. The matrix precision error, i.e., the precision error due to variability in the waste matrix from drum to drum, is estimated to be $5.5 \%$ of the true Pu quantity in the drum.

In order to be critically safe, there is a safety limit requiring that the measured $\mathrm{Pu}$ mass plus 2 times its standard error must not exceed $200 \mathrm{~g}$. Based on the equations derived in this report the measured mass must be below approximately $177 \mathrm{~g}$ in order to meet this criterion for drums containing graphite waste.

Note: This report replaces a previous report regarding the uncertainty analysis of graphite waste drum measurements (Harker et al., 1995). Since the publication of that report there have been significant changes in the manner in which the PAN system operates (e.g., the addition of mass calculations based on shift register coincidence counts) as well as changes in the simulation routines. The description of the PAN system, the structure of the uncertainty analysis, and the resulting uncertainty estimates reported herein supersede the information found in the previous report. 


\section{ACKNOWLEDGMENTS}

The authors would like to acknowledge the support of others who have made significant contributions to the production of this report. Dr. L. V. East developed the data files and data listings containing the results of previous PAN measurements pertaining to the graphite content code and background measurements. D. R. Staples used the MCNP model to calculate the basic PAN responses for the cases developed in this evaluation. P. J. Taylor and C. R. Amaro reviewed the RTR video tapes and assessed the physical condition of the graphite waste form. G. K. Tedford and the PAN assay staff provided technical support related to the RTR tapes review. G. K. Becker and Dr. L. V. East provided technical insight based on their experience with the PAN and other assay measurements. Dan Menkhaus is the project sponsor and the authors would like to thank him for his support and encouragement during the course of this evaluation. 


\section{CONTENTS}

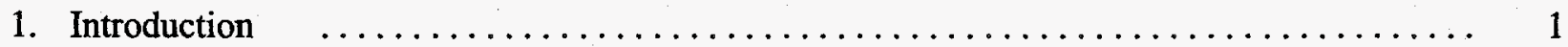

2. Passive Active Neutron (PAN) Assay System $\quad \ldots \ldots \ldots \ldots \ldots \ldots \ldots \ldots \ldots \ldots \ldots$

3. Calibration of the PAN Assay System $\quad \ldots \ldots \ldots \ldots \ldots \ldots \ldots \ldots \ldots \ldots \ldots \ldots \ldots \ldots \ldots \ldots \ldots \ldots$

4. Contributors to the Uncertainty of the Assay Results $\quad \ldots \ldots \ldots \ldots \ldots \ldots \ldots \ldots \ldots$

5. Uncertainty Evaluation Approach $\quad \ldots \ldots \ldots \ldots \ldots \ldots \ldots \ldots \ldots \ldots \ldots \ldots \ldots \ldots \ldots \ldots$

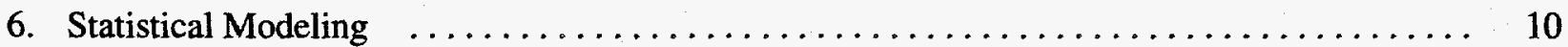

7. Simulation of the PAN Passive Mode Response $\quad \ldots \ldots \ldots \ldots \ldots \ldots \ldots \ldots \ldots \ldots$

8. Surrogate Waste Drum Benchmark Results $\quad \ldots \ldots \ldots \ldots \ldots \ldots \ldots \ldots \ldots \ldots \ldots \ldots$

9. Uncertainty Analysis Using the Latin Hypercube Sample Simulated Waste Results $\ldots \ldots \ldots 61$



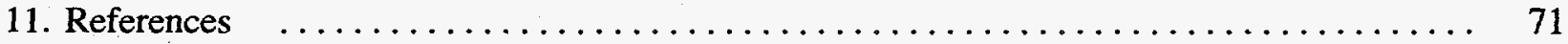

Appendix A-Spatial Variation of Density and Pu Mass Fraction $\quad \ldots \ldots \ldots \ldots \ldots \ldots \ldots \ldots$

Appendix B-Table of Parameters Required for Follow-On Calculations $\ldots \ldots \ldots \ldots \ldots \ldots \ldots$ 


\section{SWEPP PAN Assay System Uncertainty Analysis: Passive Mode Measurements of Graphite Waste}

\section{INTRODUCTION}

The Passive Active Neutron (PAN) radioassay system at the Stored Waste Examination Pilot Plant (SWEPP) is used to certify waste drums in terms of quantifying plutonium and other transuranic element activities. At this time, assay reports produced by the system provide uncertainty values which are based solely on the counting statistics of the assay technique. It is recognized that, depending on the waste form involved, there can be significant additional systematic and random errors that need to be quantified before the total assay uncertainty can be quoted.

This report presents a methodology for performing a generalized uncertainty analysis of the PAN assay system measured plutonium mass for each waste form classification. Also in this report are the results of performing this process for waste drums containing plutonium contaminated graphite waste forms (IDC code 300). While the PAN system produces measurements based on both active and three passive counts (short-gate, long-gate and shift register), only the uncertainty in the shift register passive mass value is addressed in this report.

Ideally this uncertainty evaluation would best be carried out experimentally where each parameter would be varied in a systematic way and that parameter's effect on the measured plutonium mass would be determined. Because there are a large number of parameters which can affect the assay results and some of these parameters are correlated, it is not practical to carry out a full experimental uncertainty evaluation. Therefore, a calculation/simulation approach was devised to achieve the same goal. While the general process applies equally to active interrogation and passive coincidence counting it has only been applied to passive coincidence counting at this time.

The structure of this report will focus on walking through the uncertainty analysis process for graphite waste in 208 liter drums. As backup information, a brief review of the PAN assay system is presented in Section 2. This is followed in Section 3 by a review of the original calibration of the PAN assay system performed by Los Alamos National Laboratory. Section 4 identifies the major contributors to assay uncertainties. In Section 5 the basic approach used to evaluate the PAN assay system uncertainty is described. Section 6 describes the statistical modeling involved in setting up the simulated graphite assay cases. Section 7 steps through the process of simulating the PAN passive mode response. To address the nuances associated with coincidence counting, the theory of coincidence counting as applied in the PAN assay system is also presented. The uncertainty analysis results are given in Section 8 where the simulation approach is benchmarked against measured data taken with a surrogate graphite waste drum and Section 9 which presents the findings from the analysis of 50 simulated waste drums. Section 10 summarizes the results and presents the final uncertainty assessment with regard to plutonium assay for drums containing graphite waste.

This report replaces a previous report regarding the uncertainty analysis of graphite waste drum measurements (Harker et al., 1995). Since the publication of that report, there have been significant changes the manner in which the PAN system operates (e.g., the addition of mass calculations based on shift register coincidence counts) as well as changes in the simulation routines. The description of the 
PAN system, the structure of the uncertainty analysis, and the resulting uncertainty estimates reported herein supersede the information found in the previous report. 


\section{PASSIVE ACTIVE NEUTRON (PAN) ASSAY SYSTEM}

The SWEPP PAN system is a second generation passive active neutron system developed in the early 1980's by Los Alamos National Laboratory for the U. S. Department of Energy (DOE) and delivered to the Idaho National Engineering and Environmental Laboratory (INEEL) in 1983. This system was designed to assay drums containing transuranic contaminated waste. Later a similar system was built by LANL and delivered to the INEEL for the purpose of assaying boxes containing transuranic waste. Even though much of the formalism will apply to both the drum and box assay systems, the specifics in this report will address only the drum system. The SWEPP PAN assay system is described in an INEEL internal document (Becker, 1993a); for more details the reader is referred to that document. A summary description is given below.

The PAN assay system consists of a shielding housing which surrounds the drum on all four sides, top and bottom. Each side of the housing contains moderator (i.e., graphite, polyethylene), thermal and lowenergy neutron shielding (i.e., cadmium, boron) and He-3 neutron detectors. There are two types of detector assemblies contained in each side of the assay system; i.e., bare detectors and shielded detectors.

The shielded detectors are grouped into detector packages where each package is surrounded by a thermal and low-energy neutron shield consisting of cadmium and borated rubber. Inside the cadmium and borated rubber are three or four He-3 neutron detectors surrounded by polyethylene. This type of detector assembly is sensitive to fast neutrons and insensitive to thermal and low-energy neutrons. The bare detectors are also He-3 detectors surrounded by polyethylene but are not shielded by cadmium or borated rubber. In this configuration they are sensitive to all neutrons.

The assay system operates in two modes, passive and active. In the passive mode the detector assemblies (bare and shielded) are detecting neutrons produced by spontaneous fission and $(\alpha, n)$ interactions in the waste matrix. Differentiation between the fission neutrons and the $(\alpha, n)$ neutrons is accomplished by coincidence event counting. In this type of counting, a coincidence event is recorded when two neutrons are detected by the system within a specified time window. There are two coincidence windows used: one is $35 \mu \mathrm{s}$ long and looks for coincidence events from the shielded detectors in the enclosure. This is referred to as the short-gate (SG) or shielded coincidence mode. The other gate is $250 \mu$ s long and looks for coincidence events from all "system" detectors (shielded and bare) in the enclosure. There are two coincidence circuits that use all system detectors and a $250 \mu \mathrm{s}$ gate window. The original circuit is referred as the long-gate (LG) or system coincidence mode. A recent addition has been the shift register coincidence (SRC) mode which uses signals from all system detectors and a $250 \mu$ s window.

In addition to the coincidence counting, single event counting is also accumulated during the passive mode. The single event counting data are used to derive chance coincidence corrections to the coincidence data and also to arrive at a moderator index (MI).

In the active mode the shielded detectors are used to detect neutrons produced by stimulated fission resulting from thermal neutron interrogation. The interrogation neutron source for the active mode is a Zetatron $14 \mathrm{MeV}$ neutron generator located at one corner inside the system shield enclosure. The high energy neutrons are moderated to thermal via the graphite moderator in the enclosure walls and varying amounts of moderator in the waste matrix. For the active mode the signal of interest is taken from a gated count of the shielded detectors for the time window from $700 \mu$ s to $2700 \mu$ s following each neutron burst from the neutron generator. This time window was selected to allow the fast neutrons from the generator to thermalize in the enclosure and thereby have a higher probability to stimulate fission in the $\mathrm{Pu}-239$ and at the same time the thermalized interrogation neutrons are not detectable by the shielded 
detectors. To account for background, another count window is opened from $5.7 \mathrm{~ms}$ to $15.7 \mathrm{~ms}$ after each neutron burst. It is expected that during this time window only background neutrons will be present.

Also during the active mode two monitors are used to monitor the interrogation neutron flux and the effective transmission of interrogation neutrons through the contents of the drum. The first monitor, called the cavity monitor, consists of one bare He-3 detector mounted inside the assay system enclosure on the back wall of the chamber along an upper corner. The second monitor, called the barrel flux monitor, is a single He-3 detector inside a cadmium collimator mounted at mid-height in the adjacent back corner of the assay system enclosure so that the detector's field of view is the center of the drum. These two monitors are gated with the same time window as the shielded detectors during the active mode. The ratio of the cavity monitor count during active mode to the barrel monitor count during active mode is referred to as the absorber index (AI).

The moderator index (from the passive mode count) and the absorber index (from the active mode count) are used in the analysis algorithm to arrive at correction factors which are intended to correct for moderator and absorber effects on the measured responses (both active and passive responses). The corrected responses are used to determine the measured plutonium mass. Therefore, both the active and passive counts must be completed to obtain the needed correction factors. Four measured mass values are obtained by the system for each measurement sequence (passive count + active count); i.e., a mass value determined from the active mode count, a mass value determined from the passive short-gate coincidence count, a mass value determined from the passive long-gate coincidence count, and a mass value determined from the shift register coincidence count. However, not all four values are valid over the mass range and waste forms covered in SWEPP waste. A set of selection algorithms is included in the system software to determine which of the four assay values should be used in the waste certification documentation. For most waste assays where the SRC mass is greater than $5 \mathrm{~g} \mathrm{Pu}$ (weapons grade), the SRC passive mass value is the reported $\mathrm{Pu}$ mass. For assays where the SRC mass is less than $5 \mathrm{~g} \mathrm{Pu}$, the assay system algorithm will select the active Pu mass as the reported Pu mass. In the event that the SRC mass is questionable or the SRC is not operating, the SG or LG Pu masses can be used as backup values. Under these conditions, the selection criterion for the reported passive mass is based on which of the short-gate and long-gate coincidence mass values has the smaller relative error. 


\section{CALIBRATION OF THE PAN ASSAY SYSTEM}

The basic calibration of the PAN system is performed using standard sealed neutron sources in an empty waste drum. The original calibration was performed by LANL prior to delivery of the PAN system to the INEEL. Because of changes made to the detector electronics and the addition of the shift register coincidence system, a baseline calibration was repeated in June, 1997 (Harker. 1997).

Listed below are the basic equations used by the PAN assay system to determine the mass assay values.

$$
\begin{aligned}
& \text { Mass }_{\mathrm{A}}=\mathrm{C}_{\mathrm{A}} *[(\text { Net Total Shld Adj Rate } / \text { Net Flux Mon Rate })-\text { Int. Bkgd }]_{\mathrm{A}} * \mathrm{CF}_{\mathrm{A}} * \mathrm{CF}_{\mathrm{I}} \\
& \text { Mass }_{\mathrm{lg}}=\mathrm{C}_{\mathrm{lg}} *(\text { Net LG Coincidence Rate })_{\mathrm{P}} * \mathrm{CF}_{\mathrm{lg}} * \mathrm{CF}_{\mathrm{I}} \\
& \text { Mass }_{\mathrm{sg}}=\mathrm{C}_{\mathrm{sg}} *(\text { Net SG Coincidence Rate })_{\mathrm{P}} * \mathrm{CF}_{\mathrm{sg}} * \mathrm{CF}_{\mathrm{I}} \\
& \text { Mass }_{\mathrm{src}}=\mathrm{C}_{\mathrm{lg}} *(\text { Net SRC Coincidence Rate })_{\mathrm{P}} * \mathrm{CF}_{\mathrm{sg}} * \mathrm{CF}_{\mathrm{I}} *(\text { SRC calibration adjustment })
\end{aligned}
$$

where:

Net Total Shld Adj Rate = Net total shielded adjusted rate,

Net Flux Mon Rate = Net flux monitor rate,

Int. Bkgd = Interrogation background,

Mass $_{\mathrm{A}}=\mathrm{Pu}$ mass as determined from the active mode,

Mass $_{\mathrm{g}}=\mathrm{Pu}$ mass as determined by the long-gate coincidence mode,

Mass $_{\mathrm{sg}}=\mathrm{Pu}$ mass as determined by the short-gate coincidence mode,

Mass $_{\mathrm{src}}=\mathrm{Pu}$ mass as determined by the shift register coincidence mode,

$\mathrm{C}_{\mathrm{A}}, \mathrm{C}_{\mathrm{g}}, \mathrm{C}_{\mathrm{sg}}$ are the base calibration coefficients for the active, long-gate coincidence, and shortgate coincidence modes, respectively,

$\mathrm{CF}_{\mathrm{A}}, \mathrm{CF}_{\mathrm{lg}}, \mathrm{CF}_{\mathrm{sg}}$ are the matrix correction factors for the active, long-gate coincidence, and shortgate coincidence modes, respectively,

SRC calibration adjustment is the factor applied to the long-gate base calibration coefficient to arrive at the SRC base calibration coefficient, and

$\mathrm{CF}_{1}$ is the isotopic correction factor which takes into account that the $\mathrm{Pu}-240$ (passive mode) and $\mathrm{Pu}-239$ (active mode) mass fractions may be different from those used during the base calibration.

The effects of waste matrix, etc. on the system response were estimated during the original calibration series and an algorithm for determining the correction factors was developed by LANL. The correction factors were determined empirically using surrogate waste drums in which generic materials; e.g., vermiculite, boric acid, sand and metal scraps, were used to simulate the waste matrix. The basic assumption in the development of the simulated waste was that the matrix was uniform, the source distribution was uniform and that each waste drum was filled to near the volume capacity of the drum. Over the years, there have been small changes made to the correction factor algorithm, but the basic premises; i.e., uniform matrix and uniform source distributions, have not changed. 


\section{CONTRIBUTORS TO THE ASSAY UNCERTAINTIES}

\subsection{Base Calibration}

In the base calibration, the system response is measured for a well characterized neutron source (i.e., known neutron strength and elemental and chemical composition) at specified positions in an empty waste drum. There are three primary uncertainties associated with the base calibration. The first is the uncertainty for the source strength which includes any decay corrections which are applied and the number of neutrons produced per decay. The second is the uncertainty about the elemental and chemical composition of the neutron source material. The elemental and chemical composition can significantly affect the reported neutron source strength by producing an unknown number of neutrons produced by $(\alpha, n)$ interactions in the source. The third uncertainty is the counting statistics associated with the base calibration data acquisition.

\subsection{Matrix and Source Effects}

In quantifying the estimates for systematic and random uncertainties, the major issue involves the validity of the uniform matrix and uniform source premise used in the PAN algorithm to the application of assaying a particular class of waste and what kind of errors are introduced as a result making this assumption. Listed below are the specific ways that real waste may differ from the uniform matrix and uniform source premise.

1. Source isotopic/chemical composition effects.

2. Non-uniform matrix absorption.

3. Non-uniform matrix moderation.

4. Non-uniform source distribution.

5. Variations in source particle size.

6. Significant voids in the matrix.

7. Shadow shielding of one region by high neutron absorption in another region.

8. Waste elemental composition not addressed by the calibration routine.

\section{$4.3(\alpha, n)$ Source Interference}

In addition to the matrix and source introduced errors there are also uncompensated effects resulting from $(\alpha, n)$ reactions occurring in the waste. Since the $(\alpha, n)$ reactions only produce one neutron per reaction, the coincidence counting method in the passive mode should differentiate between neutrons produced by fission (more than one neutron per fission) and neutrons produced by $(\alpha, n)$ reactions. However, the coincidence counting method will have a contribution due to accidental or chance coincidences. There are standard techniques to correct the coincidence counting data for these spurious events and these techniques work well when the chance coincidence rate is small compared to the real coincidence rate. In those cases where the $(\alpha, n)$ source strength is clearly dominant over the fissile neutron source strength (i.e., the chance coincidence rate is dominant over the true coincidence rate) there is a very large uncertainty associated with the correction for chance coincidence events.

In addition, high count rates will also lead to counting losses which are not compensated for in the simple correction applied in the assay system analysis routine. For example, the standard corrections applied for counting losses are based on the assumption of random events and are not applicable to correlated events as is the case in coincidence counting. The random event based corrections are valid when the correction 
is small but not when the counting loss is the same order of magnitude as the basic count rate. There are drums at RWMC where the neutron count rate is high enough that this circumstance applies. Under these situations the corrections are considered suspect and contribute significantly to the overall uncertainty of the measurement.

Thus $(\alpha, n)$ interference and counting losses can be sources of significant uncertainties in the assay results. In fact, recent experience has indicated that these effects can be the dominant contribution to the uncertainty of the passive assay results. 


\section{UNCERTAINTY EVALUATION APPROACH}

The large number of parameters with potential significant effects on assay quality precludes evaluating the PAN system's uncertainty by actual physical experimentation. Instead a calculation/simulation method was used. This computer intensive approach involves three main components: an input data set consisting of statistically generated waste drum and related measurement parameters, a computer model for the physical processes associated with each PAN assay measurement (neutron transport and temporal response), and a set of computer programs duplicating the PAN system's data processing routines (from which counting data and final mass values are obtained).

The statistical modeling process is described in Section 6 of this report. The neutron transport and temporal response calculations are performed using a benchmarked neutron transport model based on the Monte Carlo Neutron Photon (MCNP) transport code (Briesmeiser, 1986). The computer programs used to process the final data are described in an INEEL engineering design file (Meachum, 1997).

As mentioned in the introduction, while the PAN system produces $\mathrm{Pu}$ mass estimates based on active mode and three types of passive mode counts, this report only addresses the uncertainty in the reported shift register passive mass. The shift register mass value is always selected as the default passive mass for the PAN system graphite drum measurements and is selected automatically over the active mass for any shift register mass that is greater than $5 \mathrm{~g}$.

\subsection{Basic Steps in Performing the Uncertainty Analysis}

The basic steps in performing the uncertainty analysis are as follows:

1. Perform a content code specific review of probable causes of error in the PAN measurements to identify those factors which need to be included in the uncertainty analysis.

2. Review real-time radiography (RTR) video tapes, a data base of previous PAN assay results, and information provided by the waste shipper to establish either statistical distributions or appropriate values for factorial design settings for the parameters considered to have an effect on the assay results.

3. Develop a composite statistical model incorporating the variances and correlations of the distribution-based parameters identified in (1) and (2).

4. From the statistical model developed in (3), generate sets of simulated values for the distributionbased parameters by random sampling. Combine these values with assigned values for the factorial design variables to produce a complete set of parameter settings for each of 100 simulated waste drums.

5. For each simulated waste drum in the set from (4), apply the MCNP model of the PAN assay system to determine the basic efficiencies and temporal responses of the assay system.

6. For each case in the set from (4), input the results from the MCNP model produced in (5) and other parameters from the statistical model into the simulation routine of the assay system response to determine the basic counting data that would be produced by the PAN assay system under the conditions specified.

7. Apply the PAN assay system analysis algorithm to the set of counting data produced in (6) to determine the measured plutonium mass. Compare that mass to the mass used as input to the simulation process (i.e., the true plutonium mass).

8. Analyze the results from all the specified cases to arrive at the total uncertainty which can be assigned to the measured plutonium mass for the waste form being studied. 
As a check on the validity of the simulation approach for a particular content code, the simulation process is benchmarked against actual measurements on calibration drums. Each calibration drum is an actual drum built of known contents to approximate the matrix configuration found in a typical drum of the waste form under study. Measurements of each calibration drum using the PAN system are obtained with $\mathrm{Pu}$ sources with known properties placed in the drum. The quantities and locations of the Pu sources are varied from run to run. The calibration runs are also computer modeled using the same basic approach as that for the simulated waste drums. Comparison of the simulated PAN results to the known PAN results for the calibration runs thus provides a check on the validity of the simulation process. 


\section{STATISTICAL MODELING}

By modeling the relevant parameters affecting measurement system response, we are effectively creating simulated waste drums and associated conditions (e.g., background measurements) for input into the MCNP code and subsequent calculations. For the purpose of generating these simulated drums, parameters can be considered to be of two basic types, primary and secondary. Primary parameters are characteristics such as fill height, density, plutonium quantity, and certain background measurements whose determinations do not depend on other characteristics (although they may be correlated with one another). Secondary parameters are those which are in some way dependent on the nature of the primary parameters. This includes such characteristics as the distribution of $\mathrm{Pu}$ chunks within the drum which cannot be specified until fill height, the number of chunks, etc. are determined, and shielded $(\alpha, n)$ count which is closely related to total Pu quantity. The dependence of the secondary parameters on the primary parameter values requires that the statistical analysis and sampling be accomplished in two stages. First empirical data relative to the primary parameters are analyzed and appropriate values for the simulated drums determined. Once these values are obtained, they are incorporated as input into the analysis of the secondary parameter information so that the secondary simulated values can be calculated. The sections below describe how the values for the various parameters were obtained.

\subsection{Primary Parameters}

Primary parameters for simulated graphite drum configurations include: Mass of weapons grade $\mathrm{Pu}$, mass fraction of $\mathrm{Pu}-240$, fill height of drum, average matrix density in the drum, absorber index, background singles count rate (system and shielded), background coincidence count rate (long-gate and short-gate), background real plus accidental rate, background accidental rate, and several measures related to the total mass of $\mathrm{Pu}$ and the mass of Pu in chunks. Primary parameter values for the simulated drums were obtained by first assigning appropriate probability distributions to each parameter. These distributions were determined by analyzing appropriate sources of available empirical data. Once the probability distributions were assigned, random sampling from those distributions produces representative values for the simulated drums. Details of the analysis processes involved and specific primary parameter results for the graphite drums are given below.

Three sources of data for assigning probability distributions were utilized. Most drum characteristic data were obtained from the review of RTR video tapes of actual graphite waste drums. Tapes for a total of approximately 100 drums were reviewed (randomly selected from a total of approximately 600 drums for which RTR tapes are available). Data on absorber index values and the distribution of background measurements were obtained from the existing PAN database. Finally, shipping records from Rocky Flats were reviewed to obtain Pu-240 mass fraction information.

Note that absorber index is normally a measured parameter determined in the active phase of the PAN assay. However, the active phase was not simulated in the graphite case, so the absorber index is treated as a primary parameter for the graphite drums. While the absorber index generally depends on other characteristics related to the drum matrix, it was felt that in the case of graphite these effects are minor due to the uniformity of the material and the small amount of absorption associated with graphite. Thus, for ease of calculation, absorber index values were assigned based on analysis of the empirical data as described below, rather than trying to model its relationship to other matrix parameters directly.

\subsubsection{Probability Distribution and Correlation Analysis}

Appropriate probability distributions for each parameter were obtained by the following approach. First, histograms and/or probability plots of the empirical data described above were reviewed to determine the 
general shape of the distribution. Guided by this graphical assessment, normal, lognormal, or other standard distributions were fit to the data. Goodness of fit for each distribution was evaluated using the Shapiro-Wilk statistic (Madansky, 1988) and other statistical tests. For these tests, assessment of goodness of fit is based on the test of the null hypothesis that the data came from the specified distribution. In this case, failure to reject the null hypothesis leads to the conclusion that the data are consistent with the specified distribution. Thus, for example, when testing for a normal distribution, a high p-value suggests the data may be normally distributed, while a low p-value leads to rejection of the hypothesis that the data came from a normal distribution. Generally a p-value of .05 or greater was considered an acceptable fit, although some exceptions were made. The best fitting standard distribution was generally chosen for each parameter. If no satisfactory fit was obtained using the standard distributions, an exponential or other transformation to normality was considered. In one case, that of $\mathrm{Pu}-240$ mass fraction, the empirical distribution itself was used.

To restrict consideration of drum configurations to those combinations of parameters likely to occur in actual graphite drums, correlations among the various primary parameter values were also considered. Correlations indicate the degree of association between two sets of values. There are several ways of calculating correlation. In this case, they were assessed using the Spearman's Rho correlation coefficient (Hogg and Craig, 1978). This correlation value is more appropriate than the usual Pearson's $r$ in cases where the relationships are monotonic but not strictly linear. (Pearson's $r$ is however used in some subsequent comparisons below.) For each correlation, a significance test of the null hypothesis that the true correlation is zero was performed. For a given number of observations, the higher the observed correlation the less likely the true correlation is zero and the lower the probability will be for the significance test. Thus, low p-values, i.e., $p<.05$ indicate correlations that are statistically significant.

Results from the probability distribution and correlation analyses for the primary drum parameters are summarized in Table 6-1, Table 6-2, and Figure 6-1. Table 6-1 gives the data source, the distribution chosen, and other relevant information for each parameter. Table 6-2 lists pairs of parameters found to have significant $(\mathrm{p}<.05)$ rank order (Spearman's Rho) correlation values.

For the continuous distributions Figure 6-1 contains histograms of the primary parameter data along with plots of the fitted distributions. The histograms are plotted on the original value scale for the parameters. Since all of the distributions plotted are normal distributions or have simple transformation to normality, another indicator of the goodness of fit of the distribution to the data is obtained by considering a normal probability plot for the data (after the appropriate transformation, if any, is applied). Probability plots are inset into the histograms in Figure 6-1.

\subsubsection{Sampling Plan for Creating Simulated Primary Parameter Configurations}

Once the multivariate probability distribution for the primary waste drum parameters was determined, randomly drawn samples from this distribution produced specific simulated parameter values for the simulated graphite drums. Selecting a random sample insures that an unbiased estimate of the PAN system's overall uncertainty for this waste type is obtained. Various types of random sampling plans can be implemented, including simple random (i.e., Monte Carlo) sampling, stratified random sampling, and Latin hypercube sampling. Latin hypercube sampling (described below) is the method of choice for this type of application for two reasons. First, Latin hypercube sampling gives better estimates of mean uncertainty and uncertainty variance in the system with fewer samples. Second, sensitivity analysis (the study of the relative importance of the various input parameters) is facilitated by Latin hypercube sampling. (Response surface experimental sampling designs would actually be best for sensitivity studies, but they do not necessarily yield unbiased measures of the mean and variance of the uncertainty values--the primary aim of this study.) 
Table 6-1. Probability distributions used for LHC for physical drum parameters.

\begin{tabular}{|c|c|c|c|c|c|c|}
\hline Parameter & $\begin{array}{l}\text { Data } \\
\text { source }^{1}\end{array}$ & $\mathbf{n}$ & $\begin{array}{l}\text { Distribution } \\
\text { selected }\end{array}$ & Parameter 1 & Parameter 2 & $\begin{array}{l}\text { Goodness } \\
\text { of fit }\end{array}$ \\
\hline Density & RTR & 99 & normal & mean $=.693$ & std. dev. $=.113$ & $\begin{array}{c}\text { SW p-value } \\
=.40\end{array}$ \\
\hline Fill height & RTR & 104 & lognormal & $\begin{array}{c}\text { mean of logs } \\
\quad=3.83\end{array}$ & $\begin{array}{l}\text { std. dev. of logs } \\
=.197\end{array}$ & $\begin{array}{c}\text { SW p-value } \\
=.04^{2}\end{array}$ \\
\hline Chunks of Pu present & RTR & 97 & binomial & $p=.237$ & $q=.763$ & $\mathrm{NA}$ \\
\hline $\begin{array}{l}\text { Ratio Pu mass in chunks } \\
\text { to total } \mathrm{Pu} \text { mass }{ }^{3}\end{array}$ & RTR & 74 & lognormal & $\begin{array}{l}\text { mean of logs } \\
\quad=-2.71\end{array}$ & $\begin{array}{l}\text { std. dev. of logs } \\
=.805\end{array}$ & $\begin{array}{c}\text { SW p-value } \\
=.57\end{array}$ \\
\hline $\begin{array}{l}\text { Total Pu mass, drums } \\
\text { with no Pu chunks }\end{array}$ & RTR & 23 & lognormal & $\begin{array}{l}\text { mean of logs } \\
\quad=2.19\end{array}$ & $\begin{array}{c}\text { std. dev. of logs } \\
=.780\end{array}$ & $\begin{array}{c}\text { SW p-value } \\
=.18\end{array}$ \\
\hline $\begin{array}{l}\text { Total Pu mass, drums } \\
\text { with } \mathrm{Pu} \text { chunks. }\end{array}$ & $\overline{\text { RTR }}$ & 74 & lognormal & $\begin{array}{l}\text { mean of logs } \\
\quad=2.79\end{array}$ & $\begin{array}{l}\text { std. dev. of logs } \\
=.755\end{array}$ & $\begin{array}{c}\text { SW p-value } \\
=.98\end{array}$ \\
\hline Pu-240 mass fraction & $\begin{array}{c}\text { Rocky Flats } \\
\text { data }\end{array}$ & 38 & empirical & NA & $\mathrm{NA}$ & NA \\
\hline absorber index & $\begin{array}{l}\text { graphite } \\
\text { database }\end{array}$ & 545 & lognormal & $\begin{array}{l}\text { mean of logs } \\
\quad=.618\end{array}$ & $\begin{array}{l}\text { std. dev. of logs } \\
=.0761\end{array}$ & $\begin{array}{l}\text { SW p-value } \\
=.00^{2}\end{array}$ \\
\hline $\begin{array}{l}\text { daily background long- } \\
\text { gate coincidence rate }\end{array}$ & $\begin{array}{l}\text { background } \\
\text { database }\end{array}$ & 19 & normal & mean $=.9470$ & std. dev. $=.049$ & $\begin{array}{c}\text { SW p-value } \\
=.42 \\
\end{array}$ \\
\hline $\begin{array}{l}\text { daily background short- } \\
\text { gate coincidence rate }\end{array}$ & $\begin{array}{c}\text { background } \\
\text { database }\end{array}$ & 19 & normal & mean $=.0805$ & std. dev. $=.013$ & $\begin{array}{c}\text { SW p-value } \\
=.28 \\
\end{array}$ \\
\hline $\begin{array}{l}\text { daily background system } \\
\text { singles rate }\end{array}$ & $\begin{array}{c}\text { background } \\
\text { database }\end{array}$ & 19 & lognormal & $\begin{array}{c}\text { mean of logs } \\
=3.347\end{array}$ & $\begin{array}{c}\text { std. dev. of logs } \\
=.047\end{array}$ & $\begin{array}{c}\text { SW p-value } \\
=.58\end{array}$ \\
\hline $\begin{array}{l}\text { daily background } \\
\text { shielded singles rate }\end{array}$ & $\begin{array}{c}\text { background } \\
\text { database }\end{array}$ & 19 & lognormal & $\begin{array}{c}\text { mean of logs } \\
\quad=1.734\end{array}$ & $\begin{array}{c}\text { std. dev. of logs } \\
=.039\end{array}$ & $\begin{array}{c}\text { SW p-value } \\
=.53\end{array}$ \\
\hline $\begin{array}{l}\text { daily background real } \\
\text { plus accidental } \\
\text { coincidence rate }\end{array}$ & $\begin{array}{l}\text { background } \\
\text { database }\end{array}$ & 19 & lognormal & $\begin{array}{l}\text { mean of logs } \\
\quad=.3091\end{array}$ & $\begin{array}{l}\text { std. dev. of logs } \\
=.101\end{array}$ & $\begin{array}{l}\text { SW p-value } \\
\quad=.00^{2}\end{array}$ \\
\hline $\begin{array}{l}\text { daily background } \\
\text { accidental coincidence } \\
\text { rate }\end{array}$ & $\begin{array}{l}\text { background } \\
\text { database }\end{array}$ & 19 & lognormal & $\begin{array}{l}\text { mean of logs } \\
=-1.414\end{array}$ & $\begin{array}{c}\text { std. dev. of logs } \\
=.122\end{array}$ & $\begin{array}{c}\text { SW p-value } \\
=.97\end{array}$ \\
\hline
\end{tabular}

Footnotes:

1. $\quad$ RTR = visual review of real time radiography tapes for randomly selected graphite drums.. Rocky Flats data = tabulation of shipping manifests from Rocky Flats. Graphite database = all existing PAN system measurements for graphite drums. Background database $=$ all existing daily background measurements made since the implementation of the shift register in June of 1997.

2. Lack of fit with the Shapiro-Wilk statistic is due primarily to two outliers for fill height and high kurtosis for absorber index and the daily background real plus accidental coincidence rate(see plots of data). In these cases the indicated distributions fit better than other standard distributions and were thus chosen as the best representation of the data.

3. Calculations based on assuming that only chunks in the outer 11.148" ring of drum are visible in RTR images and that the distribution of chunks from the center to the edge of the drum was uniform. Hence, the visible number of chunks was multiplied by 1.5625 (the ratio of the total drum volume to the volume of the outer 11.148 " ring) to get the total number of chunks in the drum. Chunks were assumed to have an average mass of $0.2 \mathrm{~g}$. 
Table 6-2. Significant $(\mathrm{p}<.05)$ Spearman's Rho correlations.

\begin{tabular}{|l|l|c|}
\hline \multicolumn{1}{|c|}{ Variable 1 } & \multicolumn{1}{c|}{ Variable 2 } & Spearman's Rho \\
\hline Fill height & Density & -.56 \\
\hline Fill height & Ratio Pu chunks mass to total Pu mass & -.32 \\
\hline Total Pu mass, drums with chunks & Fill height & .26 \\
\hline Total Pu mass, drums with no chunks & Fill height & -.42 \\
\hline Total Pu mass, drums with chunks & Ratio Pu chunks mass to total Pu mass & -.78 \\
\hline $\begin{array}{l}\text { Daily background long-gate } \\
\text { coincidence rate }\end{array}$ & $\begin{array}{l}\text { Daily background real plus accidental } \\
\text { coincidence rate }\end{array}$ & .82 \\
\hline Daily background shielded singles rate & Daily background system singles rate & .75 \\
\hline Daily background shielded singles rate & $\begin{array}{l}\text { Daily background accidental } \\
\text { coincidence rate }\end{array}$ & .52 \\
\hline Daily background system singles rate & $\begin{array}{l}\text { Daily background accidental } \\
\text { coincidence rate }\end{array}$ & .37 \\
\hline
\end{tabular}

Latin Hypercube Sampling Described. A basic Latin hypercube sample is obtained as follows. Suppose we wish to form $\mathrm{n}$ simulated drum configurations based on the probability distributions of $\mathrm{q}$ different parameters. The range of each of the $\mathrm{q}$ parameters is divided into $\mathrm{n}$ equal probability intervals. The boundaries of the equal probability intervals are determined by calculating the appropriate quantiles of the underlying probability distribution for each parameter. Then one value from each interval is selected at random, according to the part of the specified probability distribution for the parameter that lies within that interval (i.e., not uniform sampling, but rather sampling relative to the specified parameter's probability distribution). The $\mathrm{n}$ values from each the $\mathrm{q}$ parameter distributions are then combined at random to form $\mathrm{n}$ sets of $\mathrm{q}$ parameter values to form the parameter configurations for the simulated drums. Collectively, the $\mathrm{n}$ sets of $\mathrm{q}$ parameter values is referred to as the Latin hypercube sample.

A major advantage of Latin hypercube sampling over simple random sampling is that, with Latin hypercube sampling, each $1 / n$ interval of each parameter's range is represented in the sample. In simple random sampling many intervals may go without representation. This is especially true with smaller samples. Sampling every interval once insures that the uncertainty results are representative of the entire range of expected values for each parameter. (Of course it does not insure that every combination of different intervals for all $q$ parameters will be represented--for $r$ intervals that would require $n=r^{q}$ samples.)

The basic approach to Latin hypercube sampling just described does not allow for any specific correlation structure among the parameters being sampled. When significant correlations between parameter values exist, as is the case for some of the primary parameters for the graphite drums, they can be induced in the Latin hypercube sample by restricting the manner in which the $n$ values on the $q$ parameters are combined to form the simulated drum configurations. Adjustments for correlation are based on the nonparametric rank order correlation coefficient (i.e., Spearman's Rho).

For this analysis, the Latin hypercube sampling was performed using a computer program developed by Iman and Shortencarier (1984). This FORTRAN 77 program allows the formation of samples from parameters following a number of standard distributions (e.g., normal, lognormal, uniform, loguniform, triangular, and beta) as well as user specified empirical distributions. Furthermore, the program allows specifying a rank order correlation matrix for the input parameters. The correlation structure from this matrix is then imposed on the Latin hypercube sample using the restricted pairing procedure of Iman and Conover (1982). 
Sample Size. Selecting an appropriate sample size for the Latin hypercube sample is not an exact calculation because of the varied uses to which the data will be put. Sampling just to obtain a good estimate of the overall bias and variability of the measurement system does not require as many samples as would sampling for sensitivity analysis or other types of analysis that in some way break down the data into smaller components. Other than the uses to which the data are intended, the primary determinant for sample size is the number of parameters being considered. The larger the number of parameters, the greater the sample size needs to be to get the same degree of representation of the population being sampled. Iman and Helton (1985) suggest a minimum sample size of $n=4 / 3 q$, where $q$ is the number of parameters, but that a value of $n$ between $2 q$ and $5 q$ may be beneficial if the model is not too expensive to run. For the simulated graphite drums, we chose $n=50$ runs. This is approximately 4 times the number of primary parameters.

It should be noted that two sets of background parameter measurements were generated. The first set was created for simulating the daily background drum measurements that get subtracted from the PAN measurements during the PAN system calculations. The other set corresponds to the background actually occurring at time of waste drum measurement (i.e., the background counts that occur during the actual drum counting process).

\subsection{Secondary Parameters}

Secondary parameters for the simulated graphite drums consist of fraction of $\mathrm{Pu}$ mass in fines, spatial distribution of $\mathrm{Pu}$ fines (by drum zone), spatial distribution of $\mathrm{Pu}$ chunks (height relative to fill height, distance from drum centerline, and angular position in drum), shielded $(\alpha, n)$ count, system $(\alpha, n)$ count, background coincidence count rate errors, and absorber index error. Due to their dependence on the primary parameter results and other factors, various methods other than simple distribution fitting and sampling were often required for the analysis of secondary parameters. Thus derivation of simulated values for each of the secondary parameters is addressed separately below.

\subsubsection{Mass of Pu in Fines}

For simulated drums for which the primary variables indicate that at least some Pu exists in chunks, the amount of $\mathrm{Pu}$ in fines was obtained by applying the formula $(1-\mathrm{rT})$ where $\mathrm{T}$ is the value of the primary parameter indicating total $\mathrm{Pu}$ amount in the drum and $\mathrm{r}$ is the primary parameter for the proportion of the total $\mathrm{Pu}$ mass attributable to chunks. For drums for which no Pu mass in chunks was indicated, the $\mathrm{Pu}$ mass in fines is the same as the total Pu mass.

\subsubsection{Spatial Distribution of Pu Fines}

The $\mathrm{Pu}$ fines were randomly distributed within a drum, according to a lognormal distribution. Specifically the following procedure was employed:

1. Generate 10,000 uniformly located sampling locations within the fill volume of the drum. (The large number of sample points was used to insure a smooth distribution of fines throughout the 24 zones used in the simulation modeling. 10,000 is probably a much bigger number than was actually needed, but the calculations were simple enough to perform on a computer that there was no penalty in using such a large number.)

2. For each of the 10,000 sampling locations, generate a random $\mathrm{Pu}$ fine amount based on a lognormal distribution with a mean equal to $1 / 10,000$ of the total drum $\mathrm{Pu}$ fines amount and a variance consistent with the Latin hypercube sample inter-drum $\mathrm{Pu}$ variance (i.e., the inter-drum variance 
calculated by combining the variances for the 10,000 sectors within each drum is the same as the inter-drum variance calculated from the drum total Pu amounts--see Appendix A for details.)

3. Add up the Pu fine amounts over the sampling locations within each of the 24 drum zones used in the MCNP modeling. Normalize the values to insure the sum over the 24 zones adds to the total Pu fines specified for the drums. (Very little normalization was required as the expected value of the sum will be the total $\mathrm{Pu}$ fines amount.)

4. Output the result for each zone as a zone specific mass fraction: (zone mass due to fines)/(total mass in zone) where the total mass for the zone is proportional to the ratio of the zone's volume to the total volume of waste in the drum.

\subsubsection{Spatial Distribution of Pu Chunks}

Total Pu mass in chunks was obtained by multiplying the primary parameter for total Pu mass by the ratio of $\mathrm{Pu}$ chunks to total Pu mass. Dividing this quantity by 0.2 and rounding up to the next integer value gives the number of chunks to be distributed in the drum (since all Pu chunks are assumed to contain 0.2 $\mathrm{g} \mathrm{Pu}$ ). The $0.2 \mathrm{~g}$ value for the chunk size was chosen from the RTR review of the graphite drums. In this review it was observed that the chunks were in the form of metal shavings and it was estimated from their shapes that the nominal amount of $\mathrm{Pu}$ per shaving was $0.2 \mathrm{~g}$.

Each chunk's position in the drum was determined by randomly assigning an angular position, a distance from the drum centerline, and a height relative to the drum fill height. Angular position and distance from the drum centerline were assumed to be uniformly distributed. The relative height in the drum was assigned a beta distribution (with parameters $\mathrm{v}=.48, \mathrm{w}=.63$ ) based on the analysis of the height positions of 220 chunks observed in the RTR review of 97 actual graphite drums. The fit of the beta distribution to the data was evaluated by a chi-square goodness of fit test which yielded a p-value of 1.0 when using 10 categories of relative fill height. This indicates a very good fit. A plot of the data and the fitted distribution is given in Figure 6-2.

\subsubsection{System and Shielded $(\alpha, n)$ Counts}

System and shielded $(\alpha, n)$ counts have strong dependencies on Pu mass and on each other. For these parameters, simulated drum values were obtained by using the empirical data to derive regression models for the relationships and then applying those models to the new simulated drum configuration data to obtain appropriate values. The regression analyses for these parameters were based on the database of 530 actual PAN system measurements.

To determine either the system or shielded $(\alpha, n)$ counts, the PAN database was queried for the total counts in each category and also the total Pu for each case. Based on the Pu mass value a neutron count due to spontaneous fission was determined using the nominal efficiency for the detector group in question. The difference between the total count and that calculated due to spontaneous fission was taken to be the count due to $(\alpha, n)$ interactions. This process was followed for all 530 PAN measurements on graphite drums found in the PAN database and the resulting calculated $(\alpha, n)$ counts were used as the database for the statistical analysis.

Figure 6-3 plots the relationship between shielded $(\alpha, n)$ counts and mass for the available PAN data. Given a mass for a simulated drum, an associated shielded $(\alpha, n)$ count value is derived by calculating the appropriate value for that mass from the regression line in Figure 6-3 and then adding to that a random component derived by evaluating the variability about the regression line. The constant regression component for a particular mass is calculated by the least squares regression equation for the data in Figure 6-3: 
shielded $(\alpha, n)$ count $=35.2+(2.74)(\mathrm{Pu}$ mass $)$.

Since the variability about the regression line is not constant for all mass values, the random factors were not obtained directly from the data in Figure 6-3, but rather by first applying a variance stabilizing transformation, approximating the distribution of deviations from the regression for the transformed data, generating random adjustments from that distribution, and then back-transforming to the original scale before adding to the results of Equation 6.1.

Variance stabilization was obtained by taking logs of both mass and shielded $(\alpha, n)$ count values for the data in Fig. 6-3. This yielded a relationship with a much more constant variance as shown in Figure 6-4. The variability about this log-log regression line was found to be approximately normally distributed with a mean of zero and a standard deviation of .48 . Hence the final shielded $(\alpha, n)$ count value was calculated by adding a value $\mathrm{e}^{\mathrm{x}}$ to the initial mass value obtained using Equation 6.1, where $\mathrm{x}$ is a random variate from a normal $(0, .23)$ distribution.

Note, in the case of nonconstant variance it is more typical to do all analysis on the transformed variables. In this case, it would entail generating both components of the shielded $(\alpha, n)$ value from the log-log transformed data (rather than using Equation 6-1 for the fixed component). Exponentiating the sum of the two results obtained on $\log -\log$ scale then gives a shielded $(\alpha, n)$ count value on the original scale. This method was in fact originally tried with these data. However, due to the large magnification effect exponentiation has on small values, this approach yielded results that were quite inconsistent with the data in Figure 6-3. The approach actually used (i.e., using the transformation approach only for the random component), although somewhat nonstandard, yields more consistent results.

Once the shielded $(\alpha, n)$ counts were generated. System $(\alpha, n)$ counts were derived based on their strong correlation with the shielded $(\alpha, n)$ values. This relationship is plotted in Figure 6-5. The regression equation for these data is:

system $(\alpha, n)$ count $=2.31+(4.91)[$ shielded $(\alpha, n)$ count $]$

The random variability about the regression line was approximated by a normal distribution with a mean of 0 and a standard deviation of 20.2. Thus system $(\alpha, n)$ counts for the simulated drum were based on adding a randomly generated value from this distribution to the value obtained from Equation. 6.2.

Due to a change in the algorithm with which the PAN analysis software computations are simulated between the previous and current uncertainty analysis for graphite waste, the subtraction of the amount calculated due to spontaneous fission described at the beginning of this section is no longer required. For this analysis, this quantity has been added back in to the values generated for the waste drum simulations. Values were also adjusted for the estimated bias in the system at that time.

\subsubsection{Waste Drum Background Measures}

The data in the daily background drum data base were collected over a multi-day period of time, so the variability obtained from sampling from distributions representing these data is characteristic of long term variations in background. To produce variability more consistent with the typical elapsed time between a waste drum measurement and its associated daily background drum measurement (usually a 
few hours at most), the waste drum background measures are generated based on deviations from the given daily background values.

Obtaining waste drum background counts from daily background values. Background rates occurring during a waste drum measurement are obtained by sampling at random from a normal distribution with a mean equal to the associated daily background drum value (after applying a correction--see below) and a standard deviation representative of the expected intra-day background variability. The standard deviation was estimated by obtaining sets of repeated measurement data on two surrogate graphite waste drums. Within each of the data sets, all data were collected on the same day. Hence the data are appropriate for examining intra-day variability.

After normalizing the intra-day replicate data to account for count time differences (one set was taken at 200 seconds, the other at 600 seconds) the intra-day variance in background counts was calculated for each of the data sets. The results were then pooled to obtain a final variance estimate for the intra-day variability. The square root of this variance is the pooled standard deviation value. The standard deviation values used are listed in Table 6-3.

Background count rates come from sources outside the detector enclosure (cosmic neutrons, neutrons from within the facility, and natural sources in the soil, etc.) or from cosmic interactions in the materials of the detector enclosure. If the cavity is either empty or has an empty drum in it there is less material in the enclosure to interact (compared to when a waste drum is being measured). The primary interaction for background sources outside the enclosure is neutron scattering and with a matrix inside the drum there is a greater probability to scatter background neutrons and thereby alter their probability of detection by the system detectors. For cosmic sources producing neutrons by interactions in the enclosure walls and enclosure contents, the presence of a matrix will also increase the probability of interaction. Therefore, it can be expected that there will be a difference between a background measurement taken with an empty drum and a background taken with a filled drum. Thus, it is appropriate to apply corrections to the mean when using daily background data from an empty drum to generate values for drums filled with graphite. These corrections, given in Table 6-3, are multipliers based on the ratio of the means for the IDC 300 surrogate graphite waste drum measurements to that for the empty drum using the intra-day data just described. (Note, the multipliers were later set to 1.0 in the analysis because it was found that setting them to 1.0 provided better agreement between calculated rates in the simulations and actual surrogate drum measurements.)

Table 6-3. Mean adjustments and standard deviations for simulating graphite waste drum background measures from daily background measures.

\begin{tabular}{|l|c|c|}
\hline \multicolumn{1}{|c|}{ Background measure } & Mean adjustment & Standard deviation \\
\hline Shielded singles rate & .995 & .128 \\
\hline System singles rate & 1.027 & .300 \\
\hline $\begin{array}{l}\text { Short-gate coincidence } \\
\text { rate }\end{array}$ & .928 & .0155 \\
\hline Long-gate coincidence rate & 1.094 & .0690 \\
\hline Real plus accidental rate & 1.149 & .291 \\
\hline Accidental rate & 1.061 & .0211 \\
\hline
\end{tabular}

Correlation of intra-day background drum measures. In generating the waste drum background data from the daily background measures, the various background values were assumed to be uncorrelated. This was done in spite of the fact that the background drum data base used to generate the daily background drum measures showed significant correlations between some of the rates. However, 
much of the association is actually due to the difference in background across days and/or drums and is not necessarily applicable to the measures being generated as intra-day deviations from daily background values.

The correlation structure for intra-day background measures was investigated using data from six different waste code types. Rather than test for intra-day correlations separately within each of the six data sets (which would yield unstable estimates due to the small sample sizes) the data were combined after mean centering each data set. Mean centering removes the effects of the mean differences from the correlations. (Variances were assumed to be equal.) For the centered data, only one significant correlation (either using Pearson's $r$ or Spearman's Rho correlation coefficients) between the various background measures was found, that between the shielded and systems singles rates. Even though significant, that correlation showed an explained variance $\left(R^{2}\right)$ of only $26 \%$. Hence it would seem safe to treat the intra-day background rates as being independent. Note that a likely explanation for the difference in correlations between daily repeat measurements and longer term time periods is that within a day the variance is mostly due to counting statistics error (i.e., the background really is not changing that much) while for the longer term the background does in fact change.

\subsubsection{Background Coincidence Count Rate Error}

Short-gate and long-gate background coincidence count rate errors are functions of the respective counts. In the previous graphite analysis (Harker et al., 1995), these functions were approximated by a linear regression analysis of the existing database of 106 background measurements. However, an approximation of the error as a function of the measured background coincidence count rate is routinely calculated in the PAN assay system analysis code for the processing and reporting of the PAN measurement results. The PAN system formula for calculating the coincidence rate errors as a function of the measured coincidence rate is now being applied to the simulated coincidence rate to obtain the corresponding errors for the waste drum simulations.

\subsubsection{Absorber Index Error}

Absorber index error was assigned as a function of the specified absorber index value. Based on an analysis of absorber index values and errors from a set of measurements on the surrogate graphite waste drum the appropriate function was determined to be:

Absorber index error $=-.005+014 *$ absorber index. 


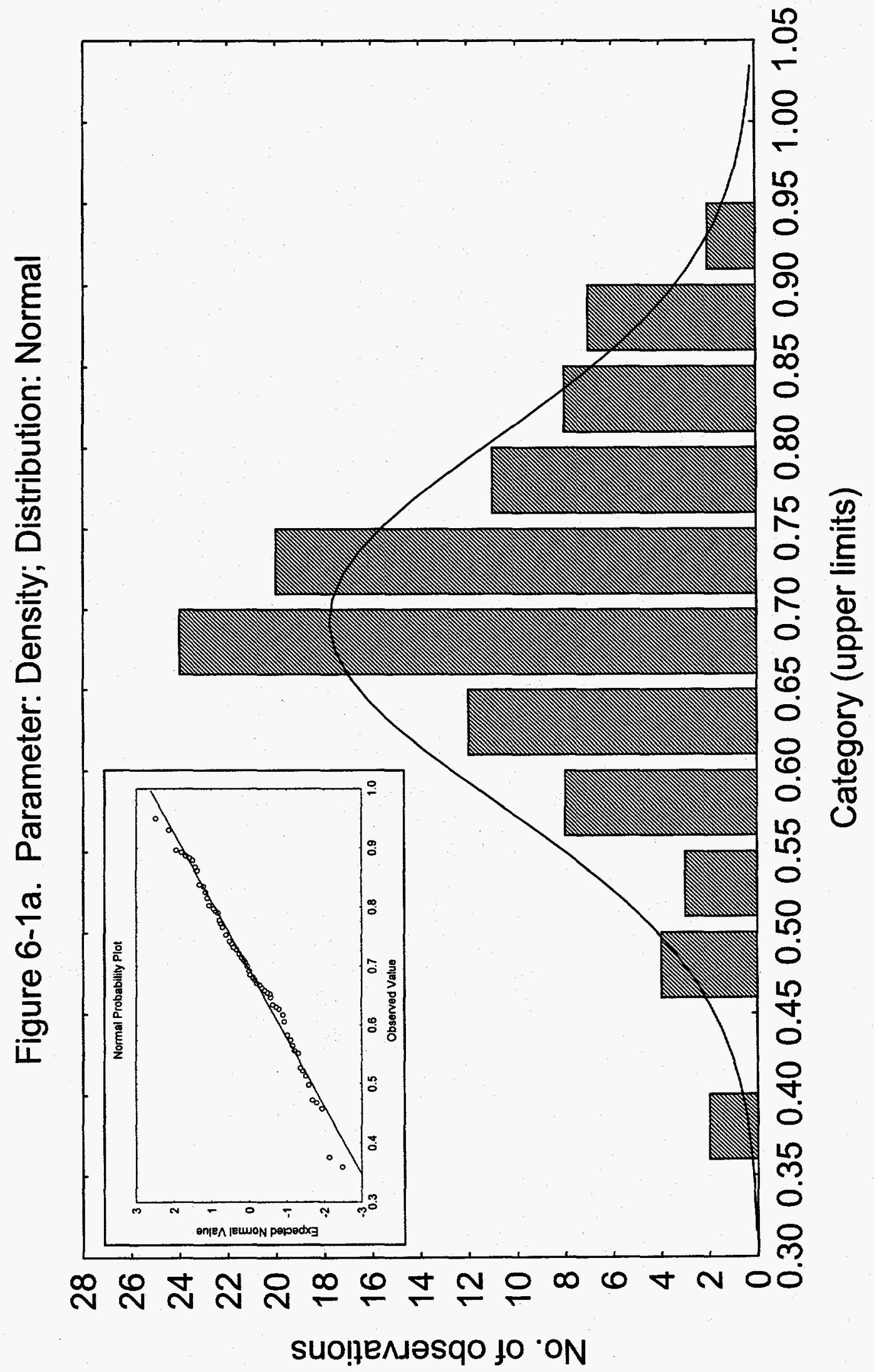




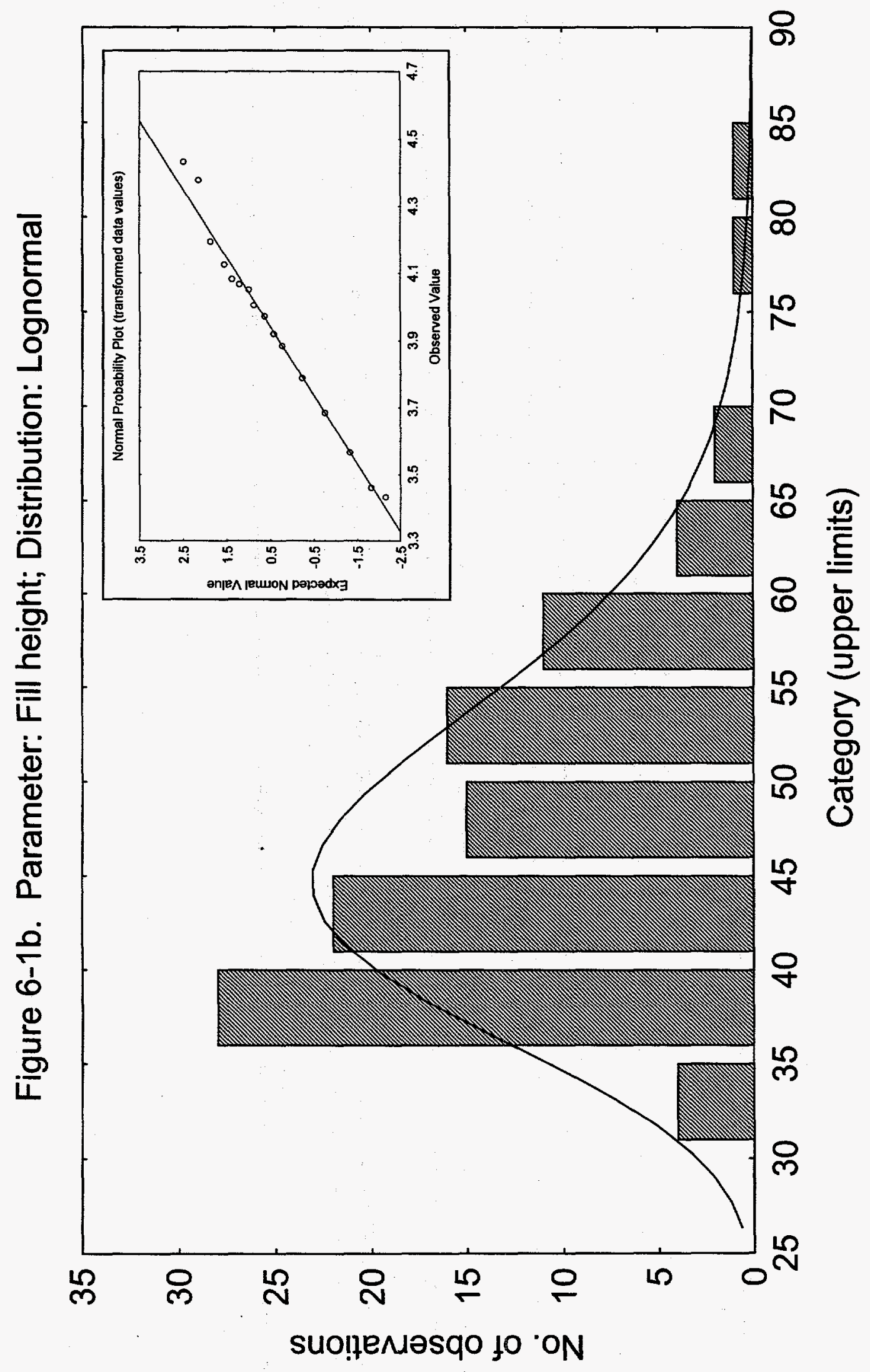


Figure 6-1c.

Parameter: Ratio Pu mass in chunks to total Pu mass; Distribution: Lognormal

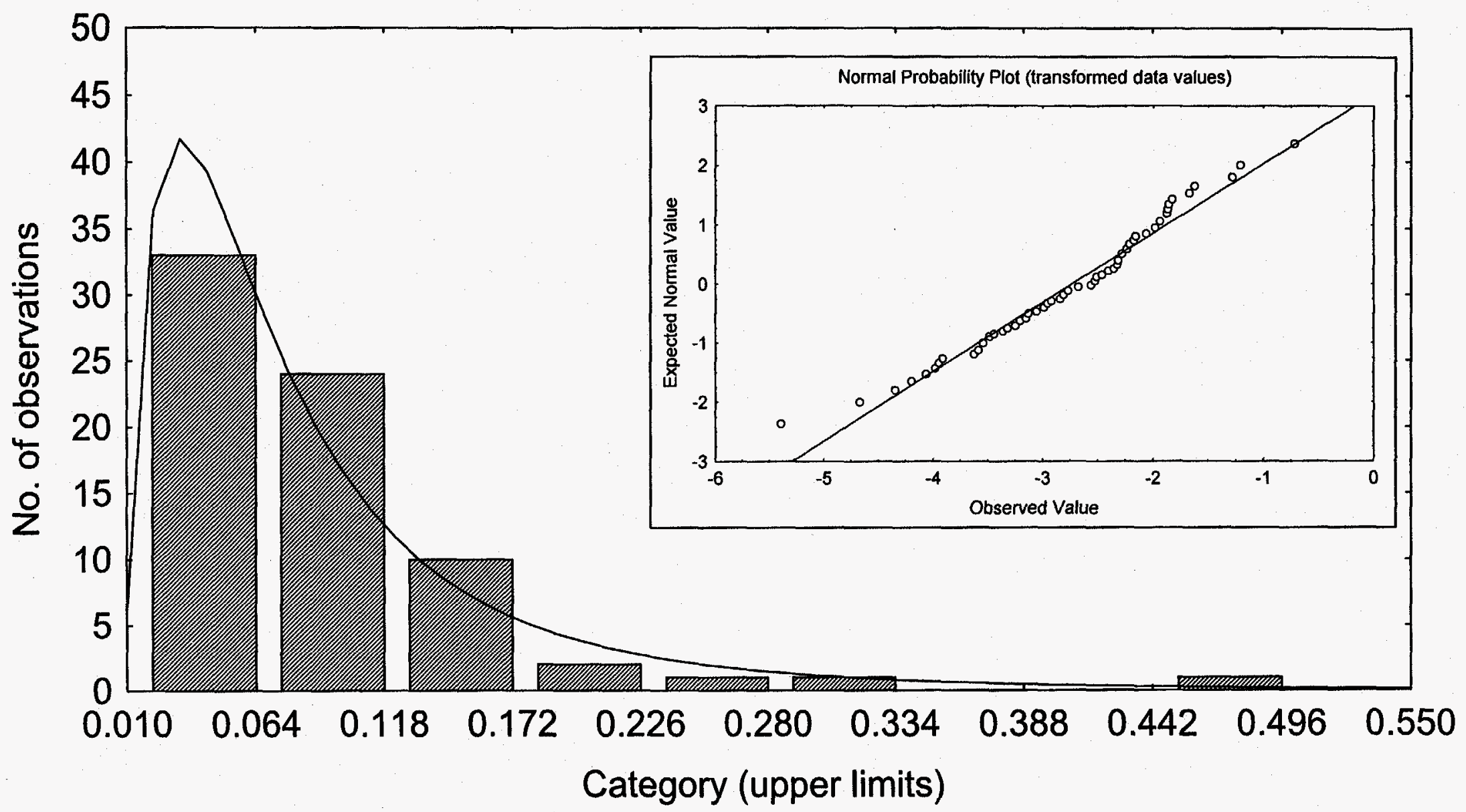


Figure 6-1d.

Parameter: Total Pu mass, drums with no chunks; Distribution: Lognormal

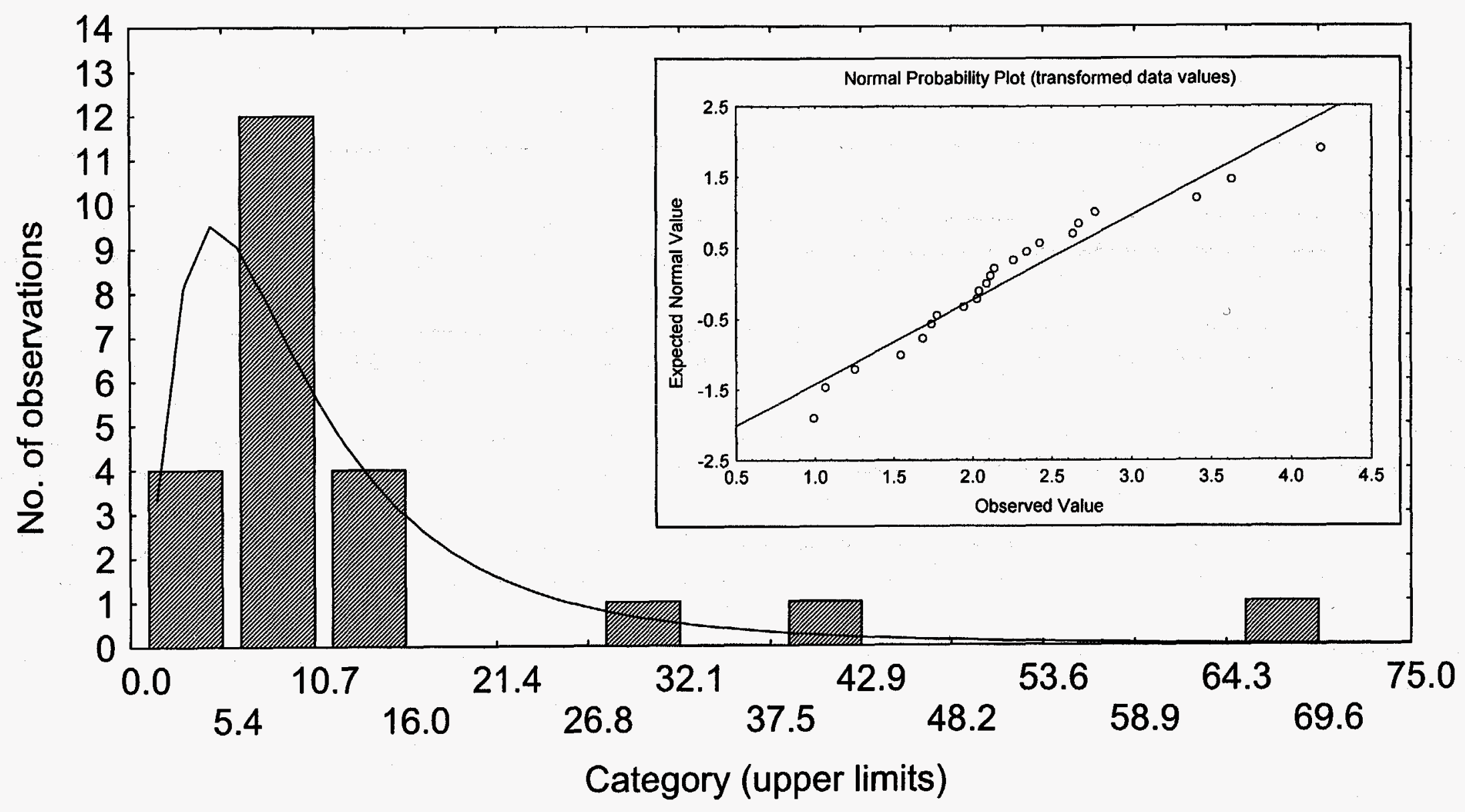




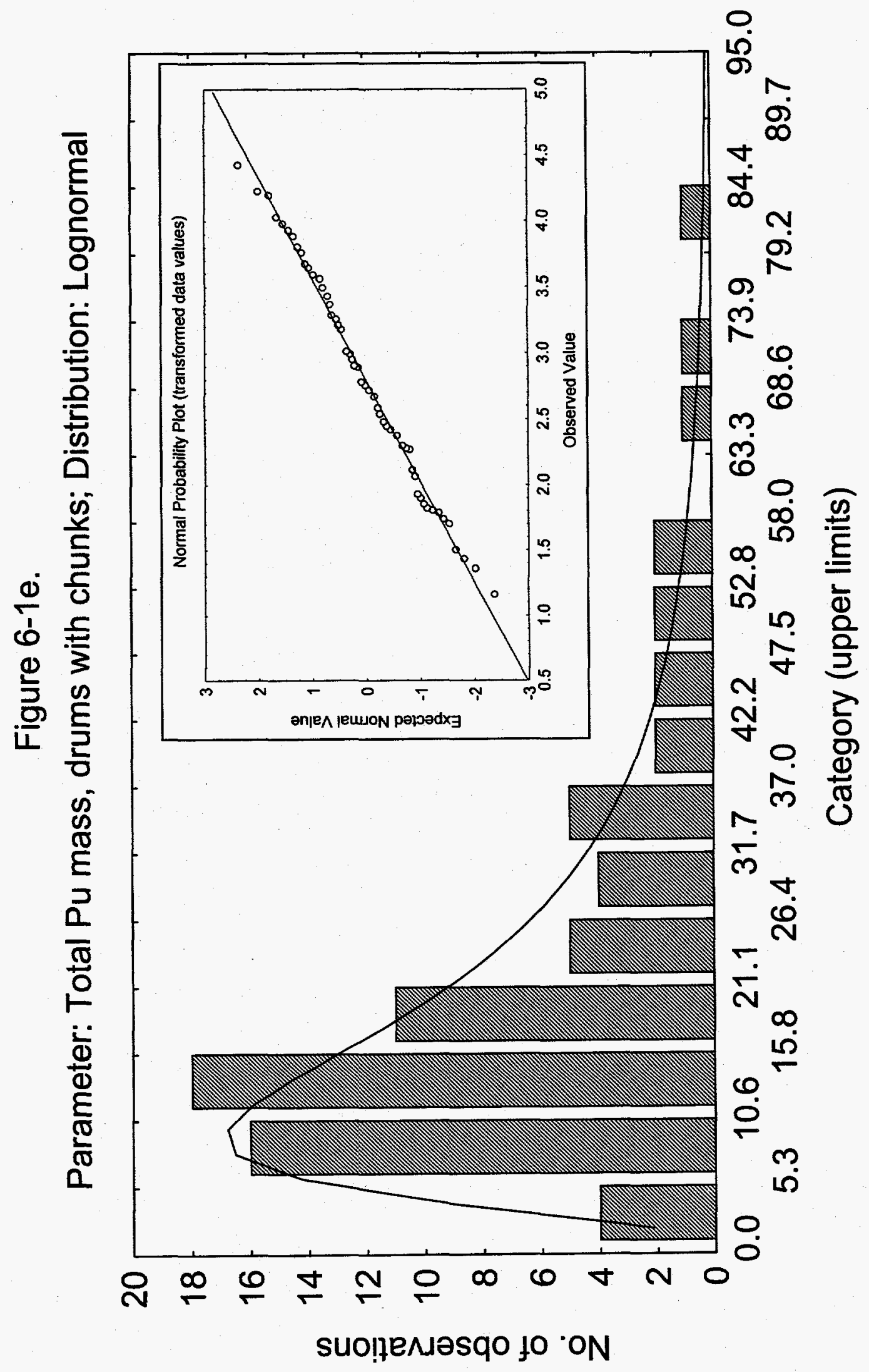


Figure 6-1f. Parameter: Pu-240 mass fraction; Distribution: Empirical

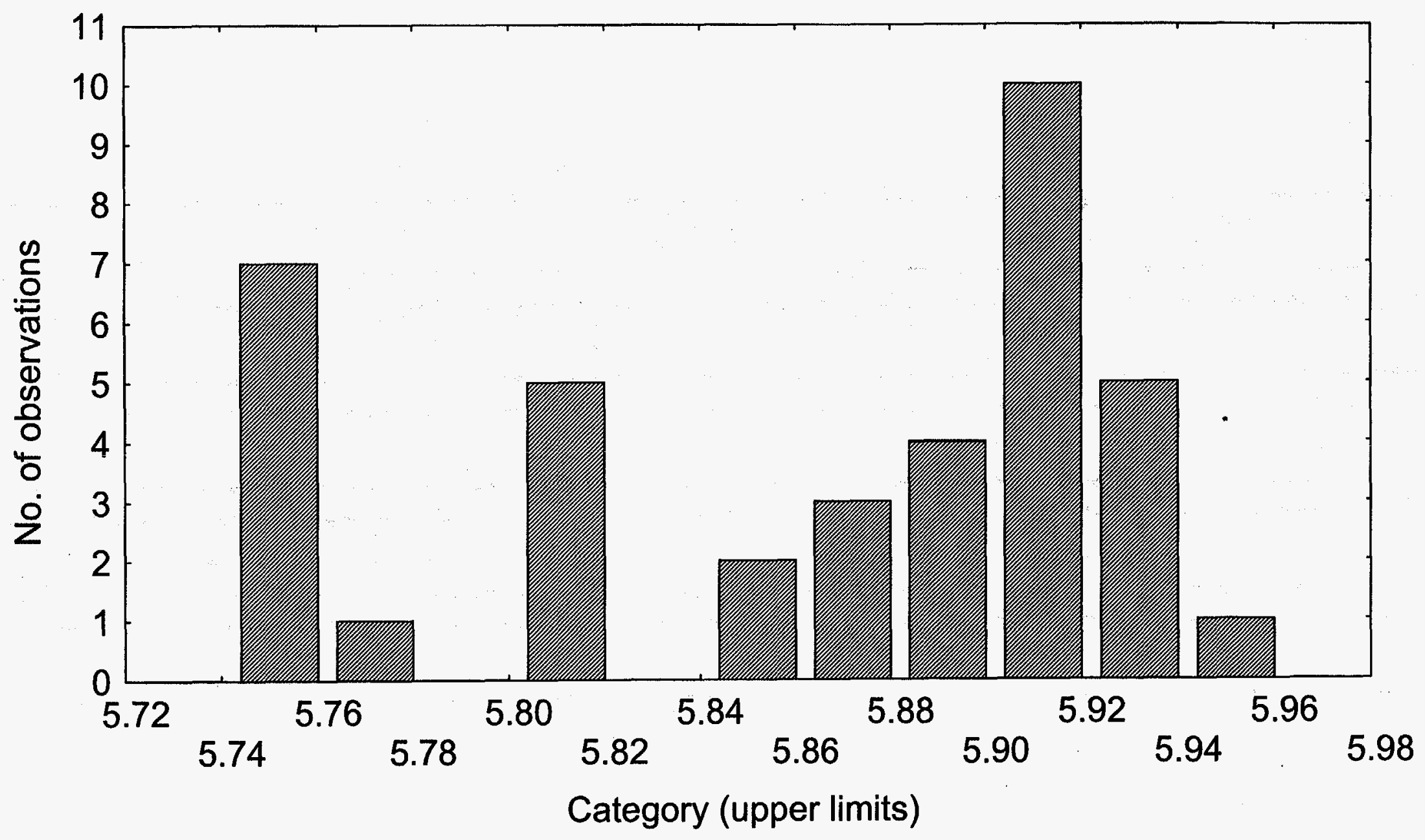




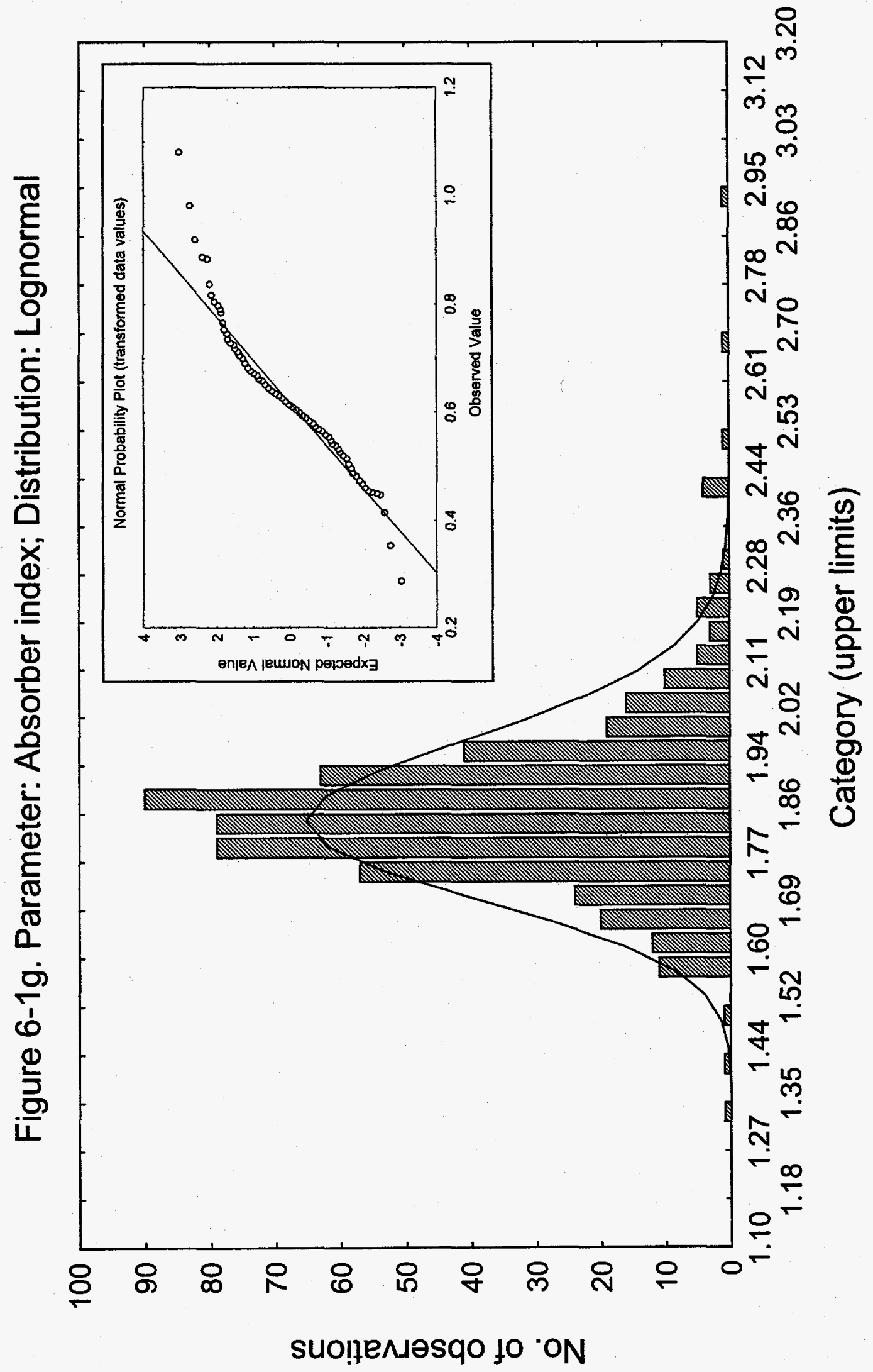


Figure 6-1h. Parameter: Daily background long gate coincidence rate Distribution: Normal

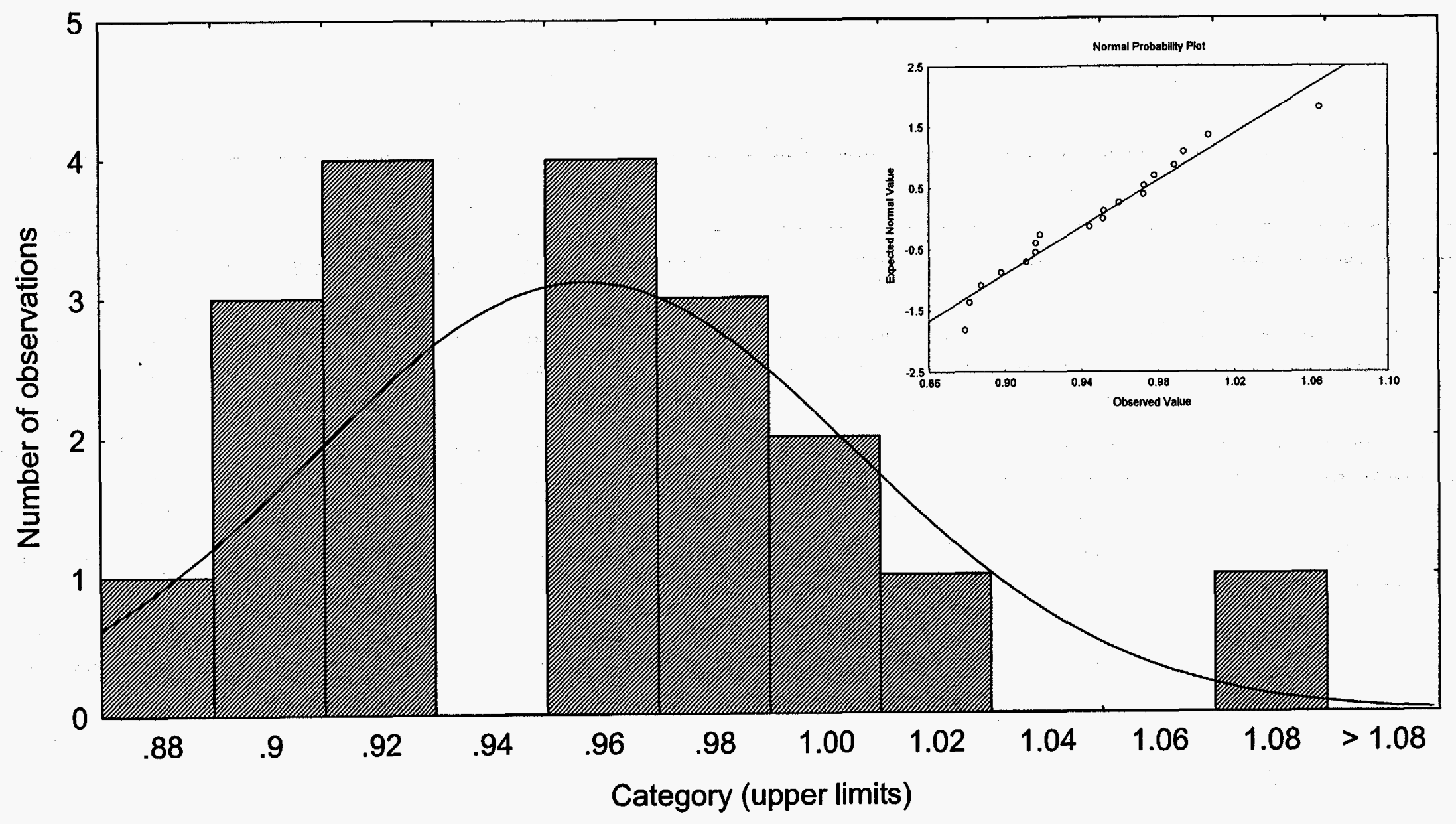




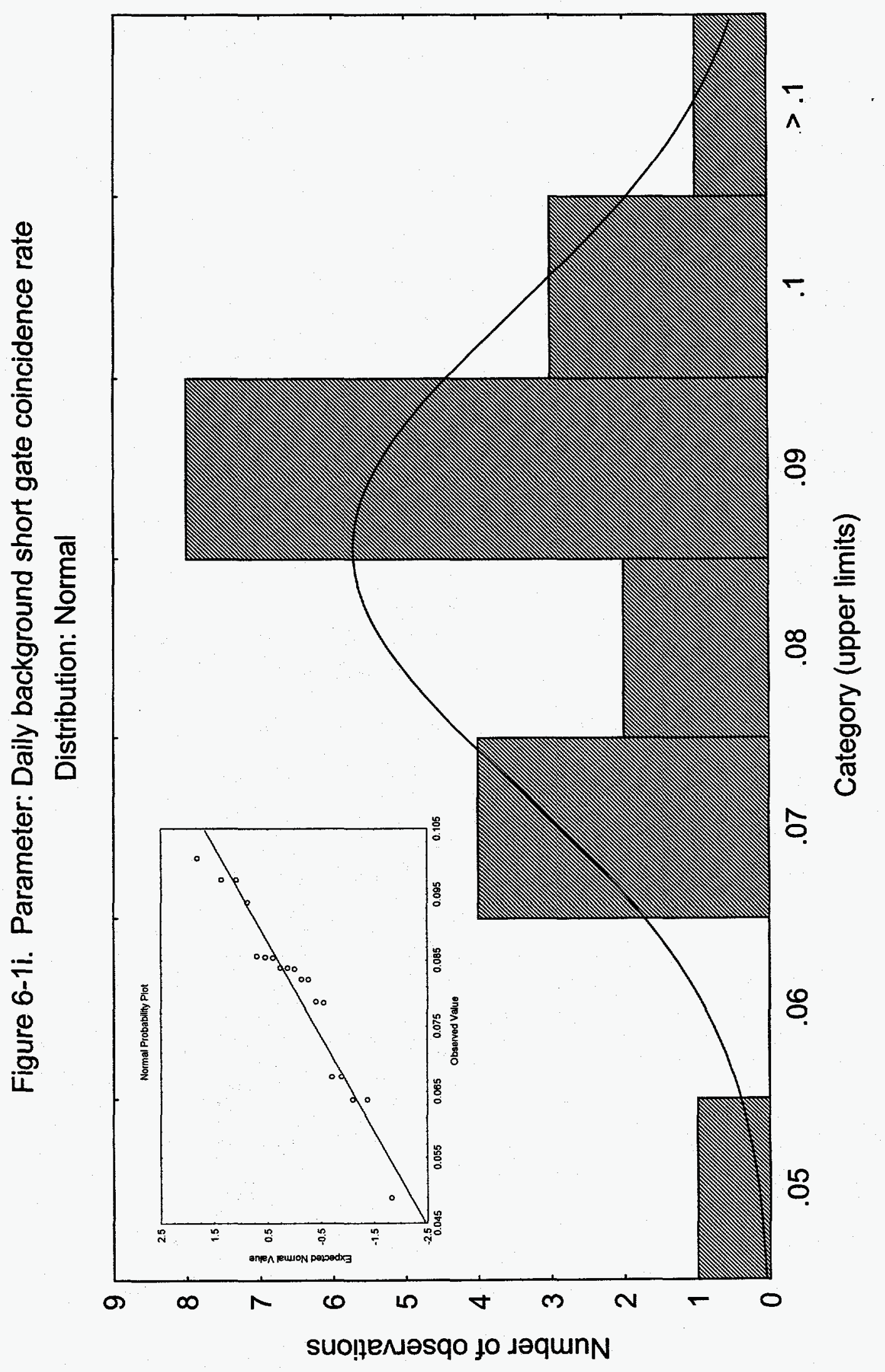


Figure 6-1j. Parameter: Daily background system singles rate Distribution: Lognormal

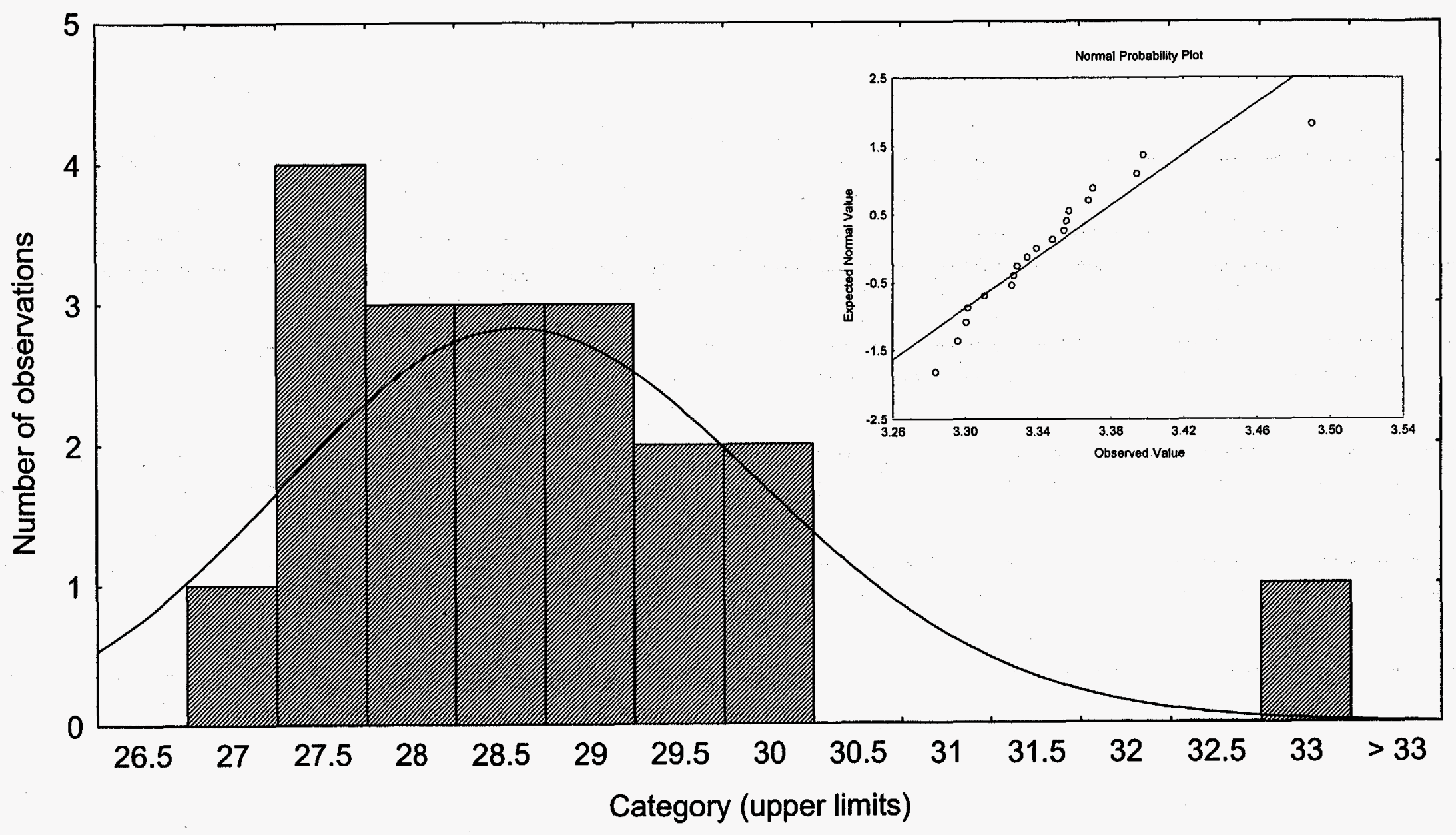




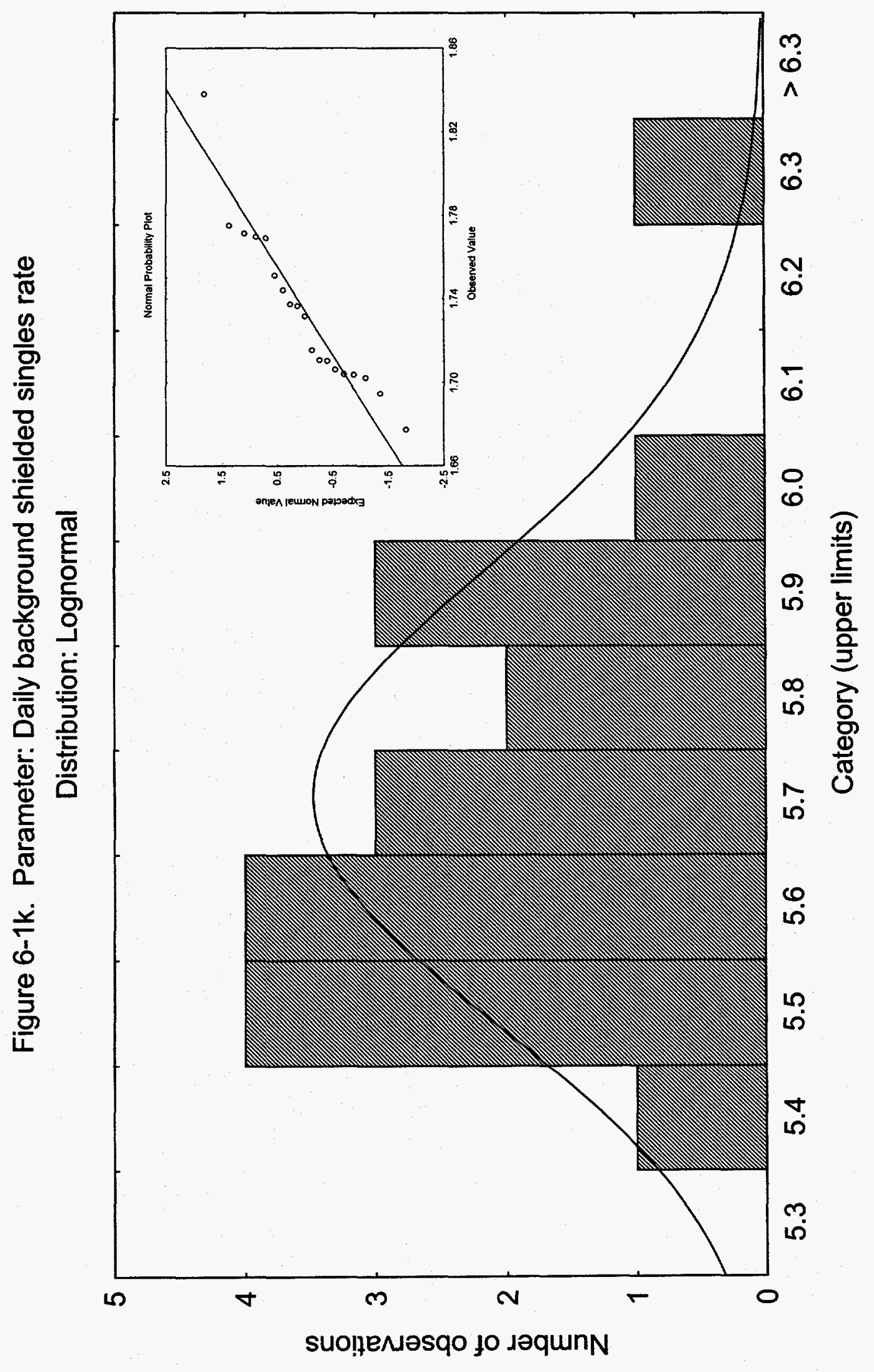




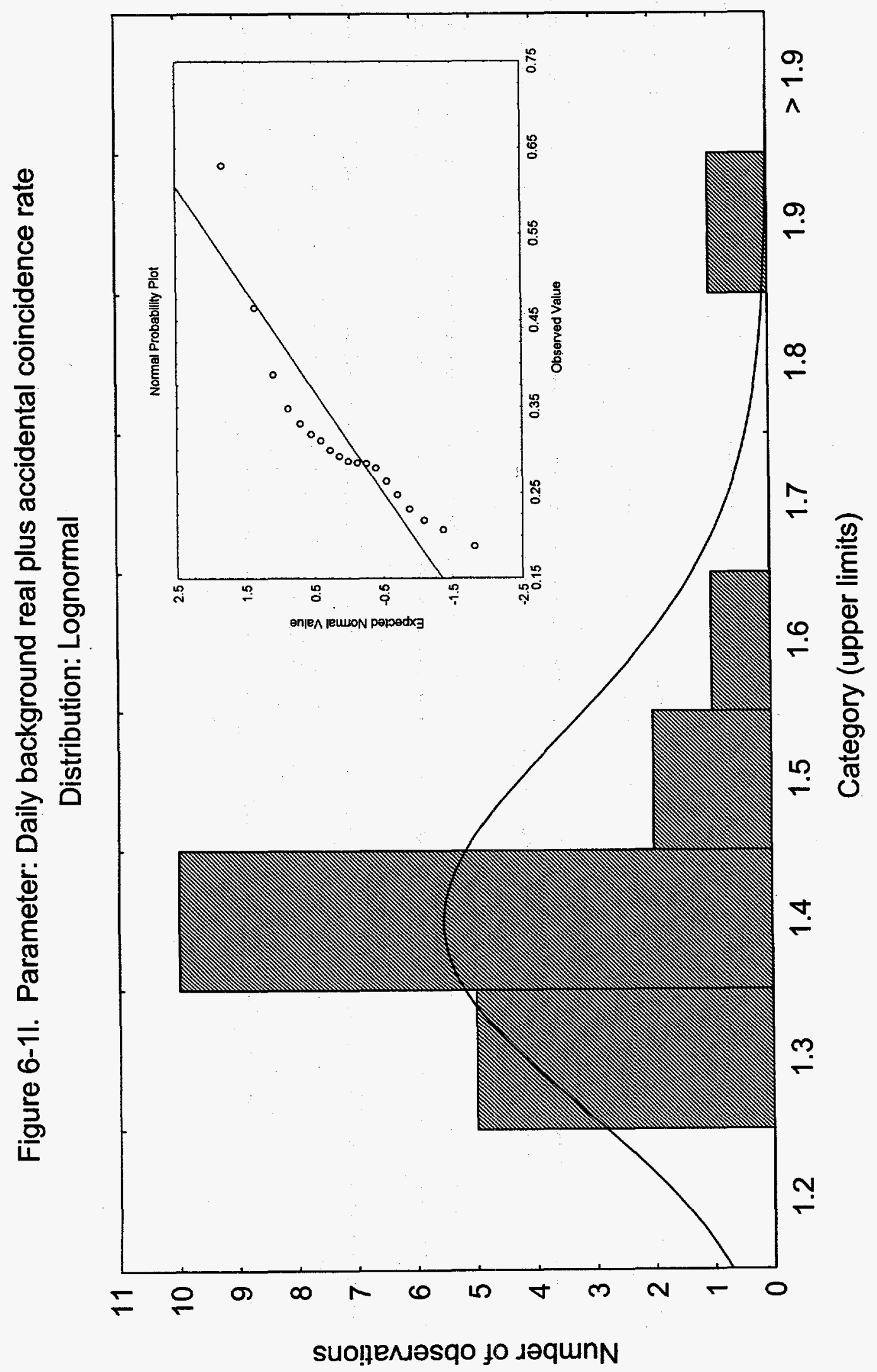




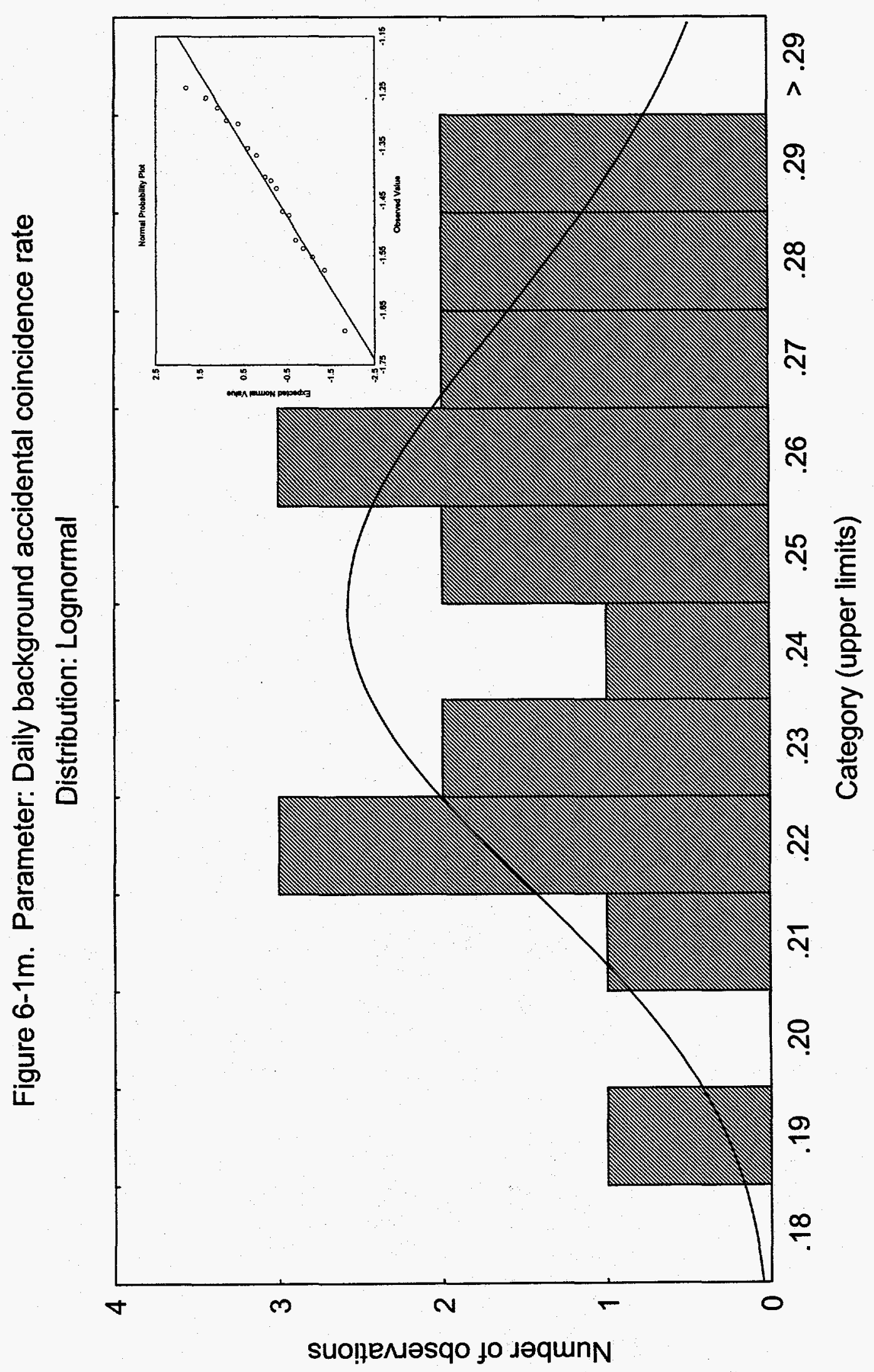


Figure 6-2. Fit of Pu chunk relative height data to a beta distribution.

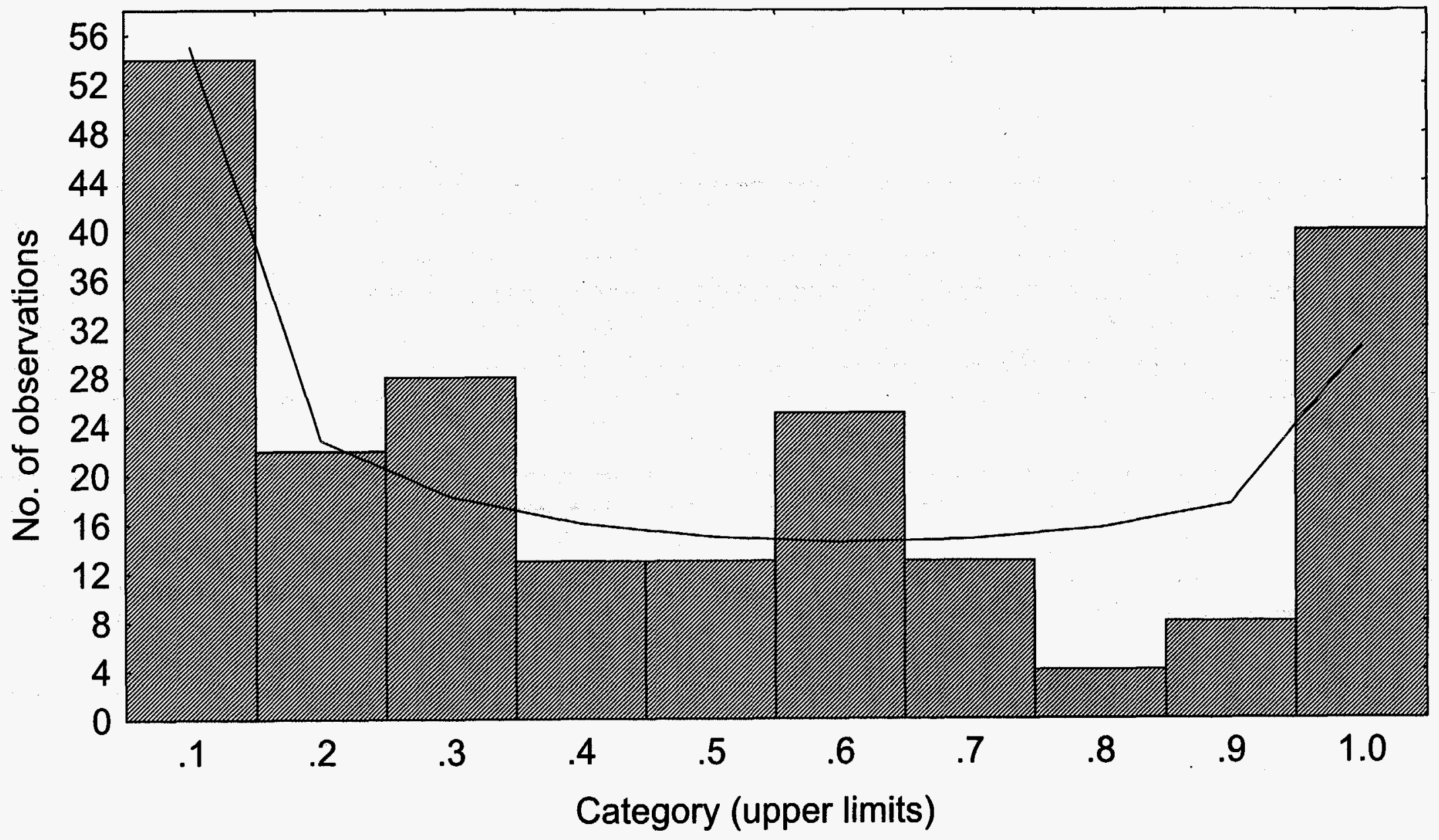




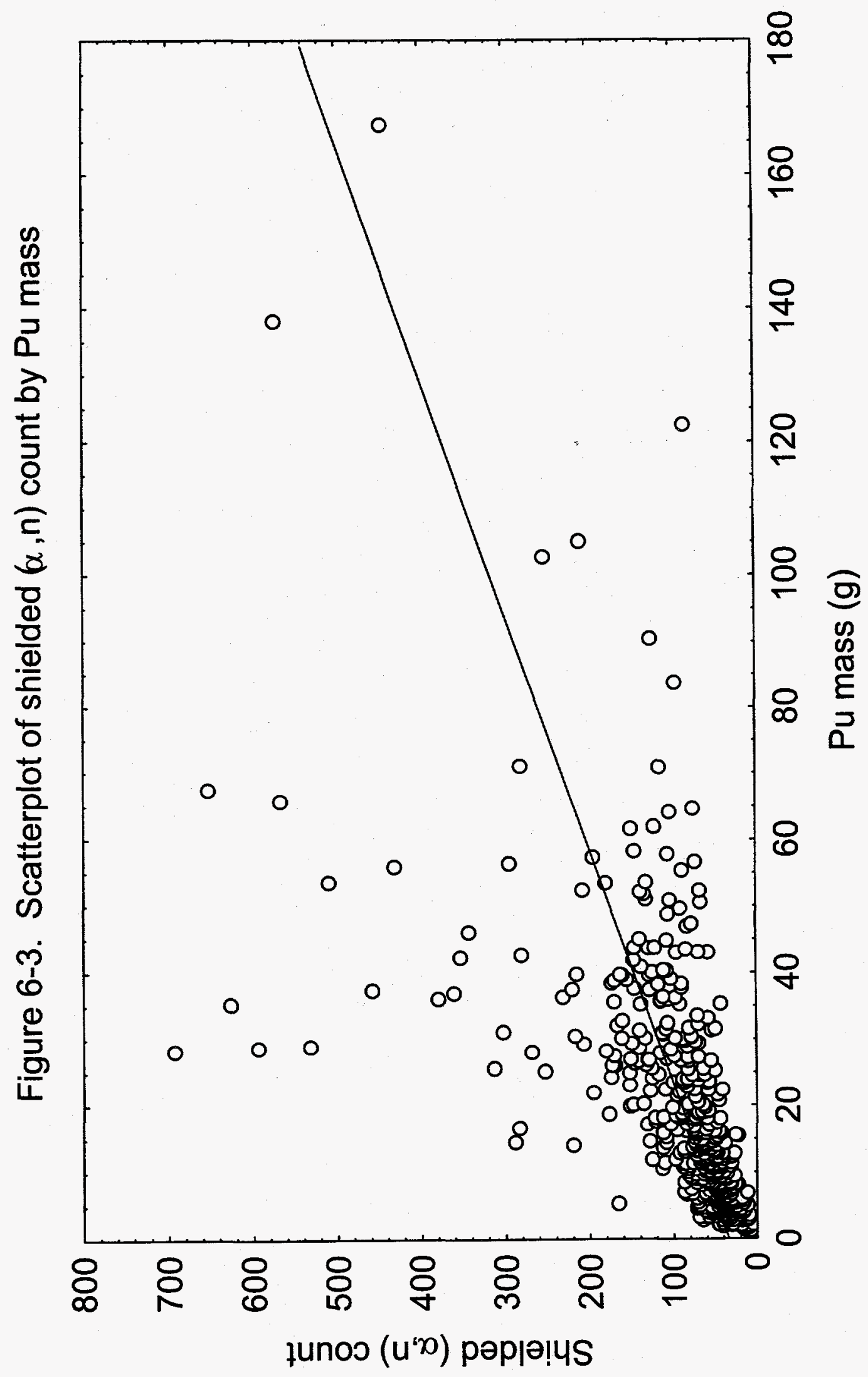


Figure 6-4. Scatterplot of log shielded $(\alpha, n)$ count by log Pu mass

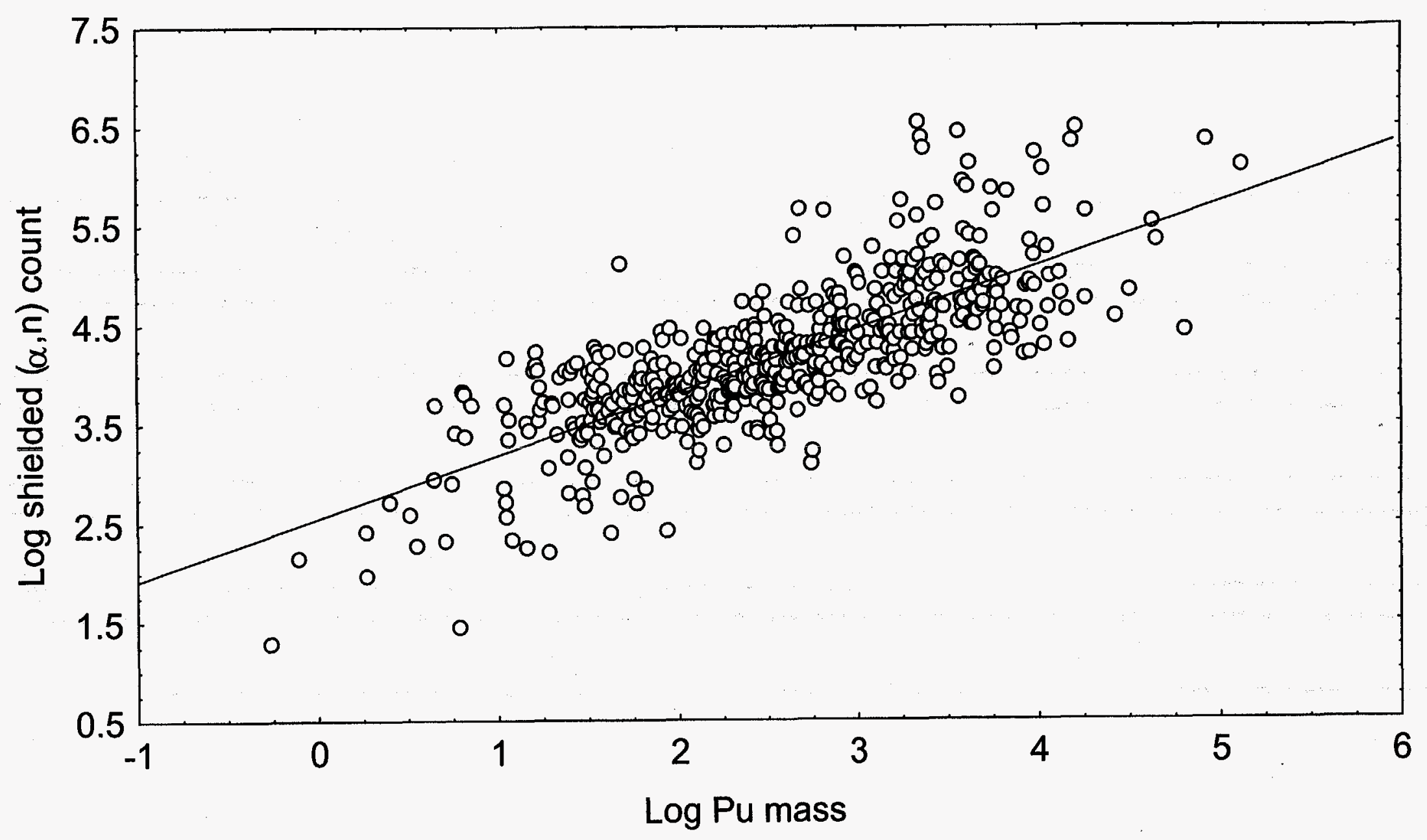




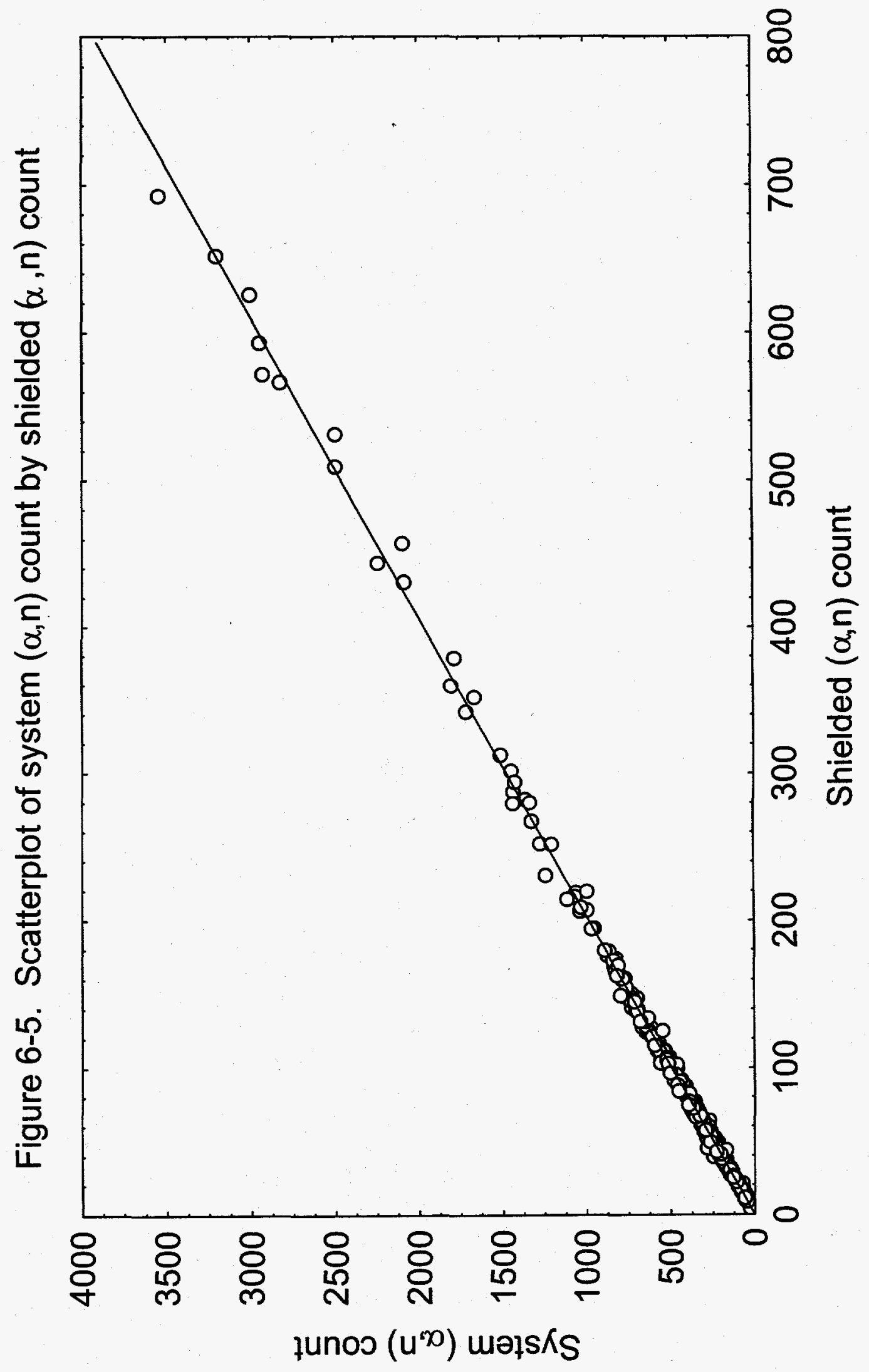




\section{SIMULATION OF PAN PASSIVE MODE RESPONSE \\ 7.1 Theory of Coincidence Counting}

The PAN assay system passive mode utilizes coincidence counting to differentiate between fission produced events where there are multiple neutrons produced and other nuclear events where only one neutron is produced per event. The type of coincidence system used in the PAN system can best be categorized as a "one-shot coincidence system." In this type of coincidence system a detected pulse opens the coincidence gate and then each pulse detected while the gate is open is counted as one coincidence event. There are two coincidence gate circuits in the PAN system, the first coincidence circuit uses the shielded detectors and is called the short-gate coincidence system. The other coincidence circuit works with all detectors (shielded plus bare detectors) and is called the long-gate coincidence system. For the short-gate coincidence system the gate time is $35 \mu$ s and for the long-gate coincidence system the gate time is $250 \mu \mathrm{s}$.

\subsubsection{Singles Count Response (Without Counting Loss Included)}

To arrive at the formulae that depict the passive response, it is necessary to start with depicting responses that do not contain counting losses. The basis for the passive response is the spontaneous fission rate of $\mathrm{Pu}-240, \mathrm{~N}_{\mathrm{f}}$.

$\mathrm{N}_{\mathrm{f}}=\lambda_{\mathrm{sf}} \mathrm{N}_{240}$

where

$\lambda_{\mathrm{sf}}$ is the spontaneous fission decay constant for $\mathrm{Pu}-240$, and

$\mathrm{N}_{240}$ is the number of atoms of $\mathrm{Pu}-240$ in the drum.

This expression assumes that $\mathrm{Pu}-240$ is the source of the spontaneous fission in the waste drum. There are also small contributions from other even mass plutonium isotopes which can be included in the spontaneous fission effect by adding similar terms or by using Pu-240 effective.

The singles count rate (no counting loss) due to fission neutrons, $\mathrm{F}$, is determined by:

$F=\varepsilon N_{f} v_{a v}$

where

$\varepsilon$ is the detection efficiency (counts per source neutron), and

$V_{\mathrm{av}}$ is the average number of neutrons produced per spontaneous fission of Pu-240.

The singles count rate (no counting loss) due to uncorrelated neutrons is denoted as $\mathrm{A}$. This term contains: (1) counts produced by $(\alpha, n)$ interactions of alpha particles from the decay of plutonium and other transuranics with primarily light nuclei (e.g., oxygen, fluorine, boron) and (2) background counts.

The total singles count rate (no counting loss) is denoted as $\mathrm{S}$.

$\mathrm{S}=\mathrm{F}+\mathrm{A}$ 


\subsubsection{Singles Count Response (With Counting Loss Included)}

The measured singles rate (with counting loss included), denoted as $\mathrm{n}$, is the true count rate, $\mathrm{S}$, multiplied by the fraction of time available for counting, which for a non-paralyzable detector system is $n=S(1-$ $\mathrm{n} \delta \mathrm{t})$.

$\mathrm{S}=\frac{\mathrm{n}}{1-\mathrm{n} \delta \mathrm{t}} \quad \mathrm{n}=\frac{\mathrm{S}}{1+\mathrm{S} \delta \mathrm{t}}$

where $\delta t$ is the dead time of the detector system. (For the PAN system $\delta t$ is $\approx 4.3 \mu \mathrm{s}$.)

\subsubsection{Coincidence Count Response (Without Counting Loss Included)}

As was the case for the singles response it is instructive to first look at the coincidence response where no counting losses have been included.

The true coincidence rate (with no counting loss) from fission, denoted as $\mathrm{C}$, is given in Equation 7.5 for a PAN type coincidence system assuming that the neutron levels decrease with a single die-away constant. Also in the PAN system there is a set delay time between the trigger and gate opening, $t_{d}$.

$$
\mathrm{C}=\lambda_{\mathrm{sf}} \mathrm{N}_{240} \varepsilon^{2} \mathrm{e}^{-\mathrm{t}_{\mathrm{d}} / \tau}\left(1-\mathrm{e}^{-\mathrm{T} / \tau}\right) \frac{1}{2} \sum_{\mathrm{v}=2} \mathrm{G}_{\mathrm{v}} v(v-1)
$$

where

$v$ is the number of neutrons produced per fission,

$\mathrm{G}_{v}$ is the statistical probability of $v$ neutrons being produced per fission,

$\mathrm{T}$ is the coincidence gate time,

$\tau$ is the die-away time of the combined (detector plus drum) system, and

$t_{d}$ is the gate delay time; i.e., the time between the generation of the coincidence gate signal and the opening of the coincidence gate. This delay has been set to be $6 \mu$ s for the PAN assay system.

For $\mathrm{Pu}-240$ spontaneous fission:

$$
\sum_{v=1} G_{v} v(v-1)=4.102
$$

Equation (7.5) is a familiar expression for a "one-shot" coincidence response for the case of a simple one component die-away system with no counting loss.

\subsubsection{Coincidence Count Response (With Counting Loss Included)}

In order to account for counting losses in the coincidence response it is necessary to start with the number of coincidence gates opened per second and account for how that rate is affected by the dead time of the coincidence system. 
The measured coincidence gate rate, $\mathrm{n}_{\mathrm{c}}$, (with counting losses included) is given by:

$\mathbf{n}_{\mathrm{c}}=\mathrm{n}\left[1-\mathrm{n}_{\mathrm{c}}\left(\mathrm{T}+\mathrm{t}_{\mathrm{d}}\right)\right]$

Substituting Equation (7.4) for $\mathrm{n}$ in Equation (7.7), $\mathrm{n}_{\mathrm{c}}$ can be rewritten as:

$\mathrm{n}_{\mathrm{c}}=\frac{\mathrm{S}\left[1-\mathrm{n}_{\mathrm{c}}\left(\mathrm{T}+\mathrm{t}_{\mathrm{d}}\right)\right]}{1+\mathrm{S} \delta \mathrm{t}}$

Equation (7.8) can be rearranged to determine $\mathrm{n}_{\mathrm{c}}$ :

$\mathbf{n}_{\mathrm{c}}=\frac{\mathrm{S}}{1+S \mathrm{~T}^{*}}$

where:

$$
T^{*}=T+\delta t+t_{d}
$$

There are two types of events counted when the coincidence window is opened. First, there is the "real event" where a trigger count which opens the gate and a count during the gate are from the same fission event. Second, there is the "accidental event" where the trigger count which opens the gate and a count during the gate are from different nuclear events occurring at random times. The real coincidence event rate is denoted as " $r$ " and the accidental coincidence event rate is denoted as " $a$ ". The accidental coincidence rate is simply the coincidence gate rate, $\mathbf{n}_{\mathrm{c}}$ times the average number of counts occurring within a gate window, $\mathrm{T}$. This is given by:

$\mathrm{a}=\mathrm{n}_{\mathrm{c}}(\mathrm{nT})=\frac{\mathrm{S}}{1+\mathrm{ST} \mathrm{T}^{*}} \frac{\mathrm{ST}}{1+\mathrm{S} \delta \mathrm{t}}$

If one assumes that $S \delta t<1$, then Equation (7.10) can be approximated by:

$\mathrm{a} \approx \frac{\mathrm{S}^{2} \mathrm{~T}(1-\mathrm{S} \delta \mathrm{t})}{1+\mathrm{ST}^{*}}$

In order to calculate the measured real coincidence rate, $r$, (with counting losses included), consider that at $t=0$ a fission event occurs producing $v$ neutrons. At a later time, $t$, one of the fission neutrons is detected producing a gate signal which following a delay, $t_{d}$, opens the coincidence gate for an open time, T.

The probability, $P_{c}$, that a coincidence gate signal is generated between $t$ and $t+d t$ is given by:

$P_{c}=n_{c} d t$

The probability that the coincidence gate above will be opened by a neutron from the fission at $t=0$ is equal to the ratio of the probability that a neutron from the fission will be detected between $t$ and $t+d t$, 
VEๆ(t)dt, to the total probability that any neutron will be detected between $t$ and $t+d t$, Sdt, (where $\eta(t)$ is the normalized die-away function for neutrons detected by the detection system) In this case we have written the die away as a general function of $t$ rather than assuming that it follows a simple exponential decay with a die-away constant, $\tau$. The probability that a neutron from the fission at $t=0$ produces a coincidence gate between $t$ and $t+d t$, denoted as $P_{g}(t) d t$, would be the product of the probability that a coincidence gate is opened times the probability that the coincidence gate was opened by a neutron from the fission.

$$
P_{g}(t) d t=\frac{v \varepsilon \eta(t) d t}{S d t} n_{c} d t=\frac{v \varepsilon \eta(t)}{1+S T^{*}} d t
$$

For a coincidence gate with an open time of $T$ and a start delay time, $t_{d}$, the number of counts in a gate triggered by a neutron from the fission at time $t$ following the fission with $v$ neutrons produced is denoted as $\mathbf{N}_{\mathrm{v}}$.

$$
N_{v}=\int_{t+t_{d}}^{t+t_{d}+T} \frac{S\left(t^{\prime}\right)}{1+S\left(t^{\prime}\right) \delta t} d t^{\prime}
$$

If $S\left(t^{\prime}\right) \delta t<1$ then Equation (7.14a) can be approximated by:

$$
N_{v}=\int_{t+t_{d}}^{t+t_{d}+T} S\left(t^{\prime}\right)\left[1-S\left(t^{\prime}\right) \delta t\right] d t^{\prime}
$$

$S\left(t^{\prime}\right)$ is the time dependent singles count rate:

$S\left(t^{\prime}\right)=\varepsilon(v-1) \eta\left(t^{\prime}\right)+S$

where $S$ is the time averaged singles count rate given by Equation (7.3). Combining Equation (7.15) with Equation (7.14b) and keeping only zero and first order terms in $\varepsilon$, the coincidence count per gate is given by:

$$
N_{v}(t) \approx \varepsilon(v-1)(1-2 S \delta t)\left(\int_{t+t_{d}}^{t+t_{d}+T} \eta\left(t^{\prime}\right) d t^{\prime}\right)+S[1-S \delta t] T
$$

The additive term on the right of Equation (7.16) is the accidental count during this gate window. The product of $\mathrm{P}_{\mathrm{g}}(\mathrm{t}) \mathrm{dt}$ times $\mathrm{N}_{\mathrm{v}}$ is the number of coincidence counts in a gate triggered by a neutron count between $t$ and $d t$ due to a fission at $t=0$.

To obtain the total number of coincidence counts for each fission with $v$ neutrons produced, one must integrate $N_{v} P_{g}(t) d t$ from $t=0$ to $t=\infty$. The integral of the first term in Equation (7.16) is the real coincidence counts per fission, $R_{v}$, and integral of the second term in Equation (7.16) is the accidental counts per fission, $A_{v}$. 
The real coincidence counts per fission is:

$$
R_{v}=\frac{v(v-1) \varepsilon^{2}(1-2 S \delta t)}{1+S T^{*}} \int_{0}^{\infty} d t \eta(t) \int_{t+t_{d}}^{t+t_{d}+T} d t^{\prime} \eta\left(t^{\prime}\right)
$$

and the accidental coincidence counts per fission is:

$$
A_{v}=\frac{\varepsilon v S T(1-S \delta t)}{1+S T^{*}} \int_{0}^{\infty} \eta(t) d t
$$

Since $\eta(t)$ is normalized, the integral in Equation (7.18) is equal to 1 and the accidental coincidence counts per fission becomes:

$$
A_{v}=\frac{\varepsilon v S T(1-2 S \delta t)}{1+S T^{*}}
$$

The average coincidence counts per fission is the weighted sum over the statistical distribution of $v$ neutrons per fission times the coincidence counts per $v$ fission. The real coincidence count per fission, $R_{\mathrm{cf}}$, is:

$$
R_{\mathrm{cf}}=\sum_{v=1} \mathrm{G}_{\mathrm{v}} \mathrm{R}_{\mathrm{v}}
$$

The accidental coincidence counts per fission, $A_{c f}$, is:

$$
A_{c f}=\sum_{v=1} G_{v} A_{v}
$$

where $G_{v}$ is the normalized probability that a fission will yield $v$ neutrons.

Substituting the expressions for $R_{v}$ and $A_{v}$ from Equations (7.17) and (7.19), respectively, into Equations (7.20a) and (7.20b) yields:

$$
\begin{aligned}
& R_{c f}=\frac{\varepsilon^{2}(1-2 S \delta t)}{1+S T^{*}} \sum_{v=1} G_{v} v(v-1) \int_{0}^{\infty} d t \eta(t) \int_{t+t_{d}}^{t+t_{d}+T} d t^{\prime} \eta\left(t^{\prime}\right) \\
& A_{c f}=\frac{\varepsilon S T(1-S \delta t)}{1+S T^{*}} \sum_{v=1} G_{v} v .
\end{aligned}
$$

The sum in Equation (7.22a) is equal to the average number of neutrons per fission, $v_{a v}$, therefore, Equation (7.22a) reduces to: 


$$
A_{c f}=\frac{\varepsilon v_{a v} S T(1-S \delta t)}{1+S T^{*}}
$$

The real coincidence rate (with counting loss included), $r$, is equal to the product of the fission rate, $N_{f}$, given in Equation (7.1) with the real coincidence count per fission, $R_{c f}$, given in Equation (7.21).

$$
\mathrm{r}=\frac{\lambda_{\mathrm{sf}} \mathrm{N}_{240} \varepsilon^{2}}{\left(1+\mathrm{ST}^{*}\right)(1+2 \mathrm{~S} \delta \mathrm{t})} \sum_{v=1} \mathrm{G}_{v} v(v-1) \int_{0}^{\infty} \mathrm{dt} \eta(\mathrm{t}) \int_{\mathrm{t}+\mathrm{t}_{\mathrm{d}}}^{\mathrm{t}+\mathrm{t}_{\mathrm{d}}+\mathrm{T}} \mathrm{dt}^{\prime} \eta\left(\mathrm{t}^{\prime}\right)
$$

The accidental coincidence rate (with counting loss), $a_{\mathrm{cf}}$, due to gates triggered by fission neutrons is equal to the product of the fission rate, $\mathrm{N}_{\mathrm{f}}$, given in Equation (7.1) with the accidental coincidence count per fission, $A_{c f}$, given in Equation (7.22b).

$$
\mathrm{a}_{\mathrm{cf}}=\frac{\lambda_{\mathrm{sf}} \mathrm{N}_{240} \varepsilon v_{\mathrm{av}} \mathrm{ST}(1-\mathrm{S} \delta \mathrm{t})}{1+\mathrm{ST}^{*}}
$$

Substituting the F from Equation (7.2) into Equation (7.24a) yields:

$$
a=\frac{S^{2} T}{\left(1+S T^{*}\right)(1+S \delta t)}
$$

In a similar fashion the accidental coincidence rate (with counting loss) due to non-fission triggered gates, $a_{n f}$, can be derived by determining the rate of non-fission triggered coincidence gates times the total coincidence count per gate. The result is:

$$
\mathrm{a}_{\mathrm{nf}}=\frac{\operatorname{AST}(1-\mathrm{S} \delta \mathrm{t})}{1+\mathrm{ST}^{*}}
$$

The sum of Equations (7.24) and (7.25) is the total accidental coincidence rate, a:

$$
\mathrm{a}=\frac{\mathrm{S}^{2} \mathrm{~T}(1-\mathrm{S} \delta \mathrm{t})}{1+\mathrm{ST}}
$$

In Equation (7.26), $\mathrm{F}+\mathrm{A}$ has been replaced by $\mathrm{S}$.

\subsubsection{Shift Register Theory}

The theory for the shift register coincidence follows the same general concepts as explained above. The major difference is that the counting loss function is given by exponentials (i.e., $\exp \left(-\delta_{l} S\right)$ for singles counting and $\exp \left(-\delta_{c} S\right)$ for coincidence counting (Reilly, 1991)). Where $\delta_{t}$ and $\delta_{c}$ are dead time parameters for the single count rate and coincidence count rate, respectively. For the SWEPP PAN system as it is currently configured, $\delta_{t}=4.3 \mu \mathrm{s}$ and $\delta_{c}=8.6 \mu \mathrm{s}$. For the shift register, the singles rate is given by:

$\mathrm{n}=\mathrm{S} * \exp \left(-\delta_{\mathrm{t}} \mathrm{S}\right)$ 
where $\mathrm{n}$ and $\mathrm{S}$ are the measured singles rate and true singles rate, respectively. (Compare with Equation 7.4 for the original coincidence systems.) The real and accidental coincidence rates are given by:

$$
\begin{aligned}
& r=\lambda_{s f} N_{240} \varepsilon^{2} \exp \left(-\delta_{c} S\right) \sum_{v=1} G_{v} v(v-1) \int_{0}^{\infty} d t \eta(t) \int_{t+t_{d}}^{t+t_{d}+T} d t^{\prime} \eta\left(t^{\prime}\right) . \\
& a=S^{2} T \exp \left(-\delta_{c} S\right)
\end{aligned}
$$

The gross real plus accidental count rate would be:

$$
\operatorname{gross}(r+a)=r+a(r+a)_{B}
$$

where $(r+a)_{B}$ is the background real plus accidental count rate: In the shift register there is a second gate delayed from the first by a set value, $D$. The counts accumulated in the register associated with the second gate is referred to as the A count and its count rate, the Arate. The expression for the Arate is:

$$
\text { Arate }=\left[\lambda_{s f} N_{240} \varepsilon^{2} \sum_{v} G_{v} v(v-1) \int_{0}^{\infty} \eta(t) d t \int_{t+t d+D}^{t+t d+D+T} \eta(t) d t^{\circ}+S^{2} T\right] \exp \left(-\delta_{c} S\right)
$$

The gross(Arate) is given by:

gross $($ Arate $)=$ Arate $+(\text { Arate })_{\mathrm{B}}$

where (Arate) $)_{B}$ is the background accidental count rate.

For the shift register, the Arate calls for a second double time integral of the die-away function, $\eta(t)$.

\subsection{Calculating the PAN Response}

There are two phases to calculating the PAN response. First, the measured count data as would be produced during the acquisition phase of a PAN passive measurement on a drum must be obtained. This process is covered in Sections 7.2.1 - 7.2.3 below. Second, the count data must be processed through the same analysis procedures as used in the data analysis phase of the PAN analysis program. This is covered in Section 7.2.4.

\subsubsection{Input Parameters for Calculating PAN Response}

The items listed in Table 7-1 below will be used to derive a set of cases to simulate the passive system response based on the equations presented in Section 7.1.

As explained in Section 2, the PAN passive method has two coincidence counting modes. One mode uses the counts coming from the shielded counters and is called the short-gate coincidence mode. The other coincidence mode uses counts coming from all detectors (called system or system total) and this coincidence mode is called the long-gate coincidence mode. Parameters a1-a11 below apply equally to both coincidence modes, but parameters a12-a15 are specific to whichever mode is being calculated. In the discussions which follow the approach being addressed is equally applicable to both coincidence 
modes and for the sake of brevity the equations have been written with subscripts depicting mode implied rather than explicitly written.

Table 7-1. Input parameters for calculating the PAN response.

\begin{tabular}{|c|l|}
\hline Parameter & \\
\hline $\mathrm{a} 1$ & Count time, $\mathrm{t}_{\mathrm{c}}$ \\
\hline $\mathrm{a} 2$ & Mass of $\mathrm{WG} \mathrm{Pu}, \mathrm{M}_{\mathrm{wg}}$ \\
\hline $\mathrm{a} 3$ & Mass fraction of $\mathrm{Pu}-240, \mathrm{MF}_{40}$ \\
\hline $\mathrm{a} 4$ & Mass fraction of $\mathrm{Pu}$ in chunks \\
\hline $\mathrm{a} 5$ & Mass fraction of Pu in fines \\
\hline $\mathrm{a} 6$ & Spatial distribution of $\mathrm{Pu}$ chunks \\
\hline $\mathrm{a} 7$ & Spatial distribution of $\mathrm{Pu}$ fines \\
\hline $\mathrm{a} 8$ & Fill height \\
\hline $\mathrm{a} 9$ & Average matrix density in the drum \\
\hline $\mathrm{a} 10$ & Spatial distribution of matrix density \\
\hline $\mathrm{a} 11$ & Elemental composition of matrix \\
\hline $\mathrm{a} 12$ & Uncorrelated neutron; i.e., $(\alpha, \mathrm{n})$, singles count rate, $\left(\mathrm{A}_{\mathrm{n}}\right)_{\mathrm{sh}},\left(\mathrm{A}_{\mathrm{n}}\right)_{\mathrm{sy}}$ \\
\hline $\mathrm{a} 13$ & Background singles count rate, $\left(\mathrm{B}_{\mathrm{s}}\right)_{\mathrm{sh}},\left(\mathrm{B}_{\mathrm{s}}\right)_{\mathrm{sy}}$ \\
\hline $\mathrm{a} 14$ & Background coincidence count rate, $\left(\mathrm{B}_{\mathrm{c}}\right)_{\mathrm{sg}},\left(\mathrm{B}_{\mathrm{c}}\right)_{\mathrm{lg}}$ \\
\hline $\mathrm{a} 15$ & Detector efficiency, $\varepsilon_{\mathrm{sh}}, \varepsilon_{\mathrm{sy}}{ }^{*}$ \\
\hline $\mathrm{a} 16$ & Normalized die-away function, $\eta_{\mathrm{sh}}(\mathrm{t}), \eta_{\mathrm{sy}}(\mathrm{t})$ \\
\hline $\mathrm{a} 17$ & Background $(\mathrm{r}+\mathrm{a})$ rate for the shift register \\
\hline $\mathrm{a} 18$ & Background Arate for the shift register. \\
\hline
\end{tabular}

* For each case identified in the statistical set, items a2-a11 are used to calculate, using MCNP (see Section 7.2.2.), the detector efficiency (number of counts per source neutron) for the shielded detectors, $\varepsilon_{\text {sh }}$, and for the shielded plus bare (system) detectors, $\varepsilon_{\mathrm{sy}}$.

\subsubsection{Monte Carlo Neutron Photon (MCNP) Calculation}

The calculational approach used in this evaluation involves the use of the MCNP transport code developed at Los Alamos National Laboratory (Briesmeiser, 1986) and a model of the SWEPP PAN assay system developed by Yoon (1993). The code and model have been verified by comparisons of the calculational responses with measured data from surrogate waste drum measurements using the PAN assay system and surrogate drums designed to represent actual waste forms. Section 8 of this report provides comparisons of measured and simulated results for the graphite content code.

The MCNP model for this application is set up to provide detector efficiencies for the shielded and system (shielded plus bare) detector banks; i.e., the number of neutron events in the detector bank per source neutron. In addition it also provides a time history of the neutron events in each detector bank following the introduction of a source neutron. However, the time history requires considerably longer computer running times and was not performed for all cases. Nevertheless, enough time histories were generated to determine that, at least for graphite, the normalized coincidence response integrals as contained in Equation 7.23 are not sensitive to the variations in the model.

The model of the detector enclosure, detector packages, etc. is the result of a dedicated effort to duplicate the system exactly in the "as built" configuration. In order to do this the PAN system was partially dismantled to identify the precise configuration of this particular system. 
Two models were used in these MCNP calculations: one for the surrogate graphite waste drum cases and the other for the Latin hypercube cases simulating actual graphite drums (see Section 9). The PAN detector enclosure, detectors, drum, and $90 \mathrm{mil}$ polyethylene liner are modeled exactly the same in both the surrogate drum model and in the Latin hypercube model. The differences between the two models are in the drum contents. In the surrogate waste drum the graphite is modeled as a uniform material with a density of $0.7959 \mathrm{~g} / \mathrm{cm}^{3}$ (Becker, 1995). In the actual surrogate waste drum the graphite is composed of a stack of graphite bricks spaced to provide an average density equal to the nominal average density found in the actual graphite drums stored at SWEPP. The fill height is $53.3 \mathrm{~cm}$ from the bottom of the drum which is the nominal fill height for the actual drums. Three source insertion tubes are included in the drum. It is in these tubes that the sources are placed in the actual surrogate drum measurements and are modeled exactly as they are placed.

In the model for the simulations of the real graphite waste drums the fill height and average density vary from case to case according to the prescriptions identified in the Latin hypercube sampling process. The graphite contents are divided into sub-regions which are stacked concentric cylindrical volumes. The diameter of the center cylinder is $11.15 \mathrm{~cm}$, the thickness of the middle and outer cylindrical shells is $11.15 \mathrm{~cm}$. The height of each vertical segment is $10.6 \mathrm{~cm}$; however, the height of the topmost segment of the matrix may be less depending on the fill height. If the drum were completely filled there would be 3 radial $\times 8$ vertical $=24$ sub-regions of matrix. For graphite these volumes are assumed to have uniform density and composition within each drum. The density is the average density specified by the Latin hypercube model and varies with each case. The composition is that defined for graphite in the surrogate drum model. The plutonium source is divided into two components; i.e., particles (chunks) and fines. The particles are assumed to be thin disks which represent Pu shavings and are modeled at the coordinates specified by the Latin hypercube model. The fines are distributed in the sub-regions according to the Latin hypercube specification.

In actual measurements the drum would be rotating. To simulate rotation, four computer runs were completed for each case in the Latin hypercube model. For each of the four runs, the drum contents are rotated $90^{\circ}$ from the orientation of the previous run. The final result for each case is the average of the four runs. An exception to this procedure occurs for cases where there are no chunks of plutonium specified. In this situation, because the fines component of the Pu source and the matrix specification are already cylindrically symmetric, only one computer run is necessary. The length of a computer run is set so that a Monte Carlo statistical error of $2 \%$ is achieved for the shielded detector bank efficiency. The corresponding statistical error for the system detector bank efficiency is less than $1 \%$.

\subsubsection{Calculation Procedure for PAN Passive Response}

The following parameters constitute the output data from the acquisition phase of a PAN passive measurement. These data constitute the input to the PAN data analysis program. This program is referred to as SWEPP Assay System (SAS) analysis code.

Count Time. Typically the count time is $\mathbf{1 2 0 0}$ seconds for a normal waste drum assay; however for the measurements using the surrogate graphite waste drum, the count time was 600 seconds. The PAN acquisition monitors the count time by a scalar count of the pulses from a $1-\mathrm{kHz}$ oscillator and so count time monitor data is the number of $1-\mathrm{kHz}$ counts and the count time is determined by dividing the $1-\mathrm{kHz}$ counts by the frequency of the oscillator. Typically the measured count time deviates from the set time by less than a second. In the simulation the set count time is used to avoid having to simulate the count time measurement. 
Raw Singles Count. The raw singles count and the standard deviation based on counting statistics for the shielded, $\left(\mathrm{S}_{\mathrm{r}}\right)_{\mathrm{sh}}$, and the system, $\left(\mathrm{S}_{\mathrm{r}}\right)_{\mathrm{sy}}$, are calculated using the following steps. To achieve a simulated singles count with deviations consistent with counting statistics, the terms true singles count and standard deviation are calculated in steps 1-7 and the simulated measured singles count is calculated in step 8 below. (Note: These steps are the same for both the shielded and system responses and the subscripts "sh" and "sy" are implied depending on the response being calculated.)

1. Use Equation (7.33), $\mathrm{M}_{\mathrm{wg}}$ (a2) and $\mathrm{MF}_{40}$ (a3) to calculate $\mathrm{N}_{240}$.

$$
\mathrm{N}_{240}=\frac{\mathrm{MF}_{40} \mathrm{M}_{\mathrm{wg}}(\mathrm{g}) 6.022 \times 10^{23}}{240.054}
$$

$v_{\mathrm{av}}=2.16$ neutrons per spontaneous fission of $\mathrm{Pu}-240$

$\lambda_{\mathrm{sf}}\left(\mathrm{s}^{-1}\right)=\frac{0.69315}{\left(1.16 \times 10^{11} \mathrm{yr}\right)\left(3.156 \times 10^{7} \mathrm{~s} / \mathrm{yr}\right)}=1.893 \times 10^{-19} \mathrm{~s}^{-1}$

The spontaneous fission half life and the average number of neutrons per spontaneous fission for $\mathrm{Pu}-240$ are from NUREG-CR-5550. Using these constants the fission neutron emission rate per gram of $\mathrm{Pu}-240$ is $1026 \mathrm{n} / \mathrm{s}$.

2. Use Equation (7.2), $\mathrm{N}_{240}, \lambda_{\mathrm{sf}}, \mathrm{V}_{\mathrm{av}}$, and $\varepsilon(\mathrm{a} 15)$ to calculate $\mathrm{F}$.

3. Use Equation (7.34), $A_{n}(a 12)$, and $B_{s}(a 13)$ to calculate $A$.

$$
A=A_{n}-F+B_{s}
$$

4. Use Equation (7.3), F and A to calculate S.

5. Assume $\delta t=5 \mu$ s, use Equation (7.4) and $S$ to calculate $n$.

6. Use Equation (7.35), $\mathrm{t}_{\mathrm{c}}$ (a1) and $\mathrm{n}$ to calculate the true raw singles count (no counting statistics), $\left(S_{\mathrm{r}}\right)^{*}$.

$\left(S_{\mathrm{r}}\right)^{*}=\mathrm{nt}_{\mathrm{c}}$

7. Use Equation (7.36), to calculate the standard deviation, $\left(\sigma_{\mathrm{ss}}\right)^{*}$, of the true raw singles count, $\left(\mathrm{S}_{\mathrm{r}}\right)^{*}$.

$$
\left(\sigma_{\mathrm{sr}}\right)^{*}=\sqrt{\left(\mathrm{S}_{\mathrm{r}}\right)^{*}}
$$

8. Use Equation (7.37), to calculate the simulated measured raw singles count (with counting statistics), $S_{\mathrm{r}}$.

$$
S_{\mathrm{r}}=\left(\mathrm{S}_{\mathrm{r}}\right)^{*}+\delta_{\mathrm{s}}
$$


where: $\delta_{\mathrm{s}}$ is a statistical variant based on a Poisson distribution with a standard deviation $\left(\sigma_{\mathrm{sr}}\right)^{*}$.

Coincidence Gate Time. For the short-gate coincidence, the gate time, $T_{\mathrm{sg}}$, is set in the PAN system at $35 \mu \mathrm{s}$ and for the long-gate and shift register coincidence, the gate time, $\mathrm{T}_{\mathrm{lg}}$ and $\mathrm{T}_{\mathrm{src}}$, are set at $250 \mu \mathrm{s}$. These gate times are also monitored by the PAN acquisition system counting pulses in a short-gate scalar and in a long-gate scalar from a $1 \mathrm{MHz}$ oscillator during the time the corresponding gate is opened for coincidence counting. The measured gate times have been shown to be very consistent with the specified values and variations in gate times are not significant in determining the deviations in the system response. Therefore, in this simulation the specified gate times have been used.

Number of Coincidence Gates. The delay following a detector pulse before the opening of the corresponding coincidence gate, $t_{d}$, has been set in the PAN system at $6 \mu$ s for both the short-gate and long-gate coincidence modes. It is assumed that the typical detector dead time for processing a pulse (i.e., $5 \mu \mathrm{s}$ ) can be used to determine the small counting loss in single pulse acquisition. The following steps are used to calculate the number of coincidence gates for the short-gate, $\left(\mathrm{N}_{\mathrm{c}}\right)_{\mathrm{sg}}$, and for the longgate, $\left(\mathrm{N}_{\mathrm{c}}\right)_{\mathrm{lg}}$. Once again the subscripts "sg" and "lg" are implied.

1. Use Equation (7.9), $\mathbf{T}, \mathrm{t}_{\mathrm{d}}, \delta \mathrm{t}$, and $\mathrm{S}$ to calculate $\mathbf{n}_{\mathrm{c}}$.

2. Use Equation (7.38), $\mathrm{n}_{\mathrm{c}}$, and $\mathrm{t}_{\mathrm{c}}$ (a1) to calculate number of coincidence gates, $\mathbf{N}_{\mathrm{c}}$.

$$
\mathrm{N}_{\mathrm{c}}=\mathrm{n}_{\mathrm{c}} \mathbf{t}_{\mathrm{c}}
$$

Raw Coincidence Count and Standard Deviation. In the simulation, to achieve the raw coincidence count with counting statistical deviations included the terms true raw coincidence count and standard deviation are calculated in steps 1-4 below. Then the true values are used to calculate the simulated measured raw coincidence count and standard deviation(step 5).

1. Use Equation (7.23), Equation (7.6), $\lambda_{s f}, N_{240}, \varepsilon$ (a15), $S, T, T^{*}, \delta t, t_{d}$ to calculate the real coincidence rate, $r$, where for the graphite content code and the short-gate coincidence mode:

$$
\begin{aligned}
\int_{0}^{\infty} d t \eta(t) \int_{t+t_{d}}^{t+t_{d}+T} d t^{\prime} \eta\left(t^{\prime}\right) & =0.2873 & & \text { for the graphite surrogate drum and } \\
& =02867 & & \text { for graphite LHC samples. }
\end{aligned}
$$

and for the long-gate coincidence mode:

$$
\begin{aligned}
\int_{0}^{\infty} d t \eta(t) \int_{t+t_{d}}^{t+t_{d}+T} d t^{\prime} \eta\left(t^{\prime}\right) & =0.330 & & \text { for graphite surrogate drum and } \\
& =0.332 & & \text { for graphite LHC samples. }
\end{aligned}
$$

2. Use Equation (7.26), S, T, $\mathrm{T}^{*}$, and $\delta \mathrm{t}$ to calculate the accidental rate, a.

3. Use Equation (7.39), $\mathrm{r}$ (Equation 7.23), a (Equation 7.26), $\mathrm{t}_{\mathrm{c}}$ (b.1), and $\mathrm{B}_{\mathrm{c}}$ (a.13) to calculate true raw coincidence count (without counting statistics included), $\left(\mathrm{C}_{\mathrm{r}}\right)^{*}$. 


$$
\left(C_{r}\right)^{*}=\left(r+a+B_{c}\right) t_{c}
$$

4. Use Equation (7.40), to calculate the true standard deviation $\left(\sigma_{\mathrm{cr}}\right)^{*}$.

$$
\left(\sigma_{\mathrm{cr}}\right)^{*}=\sqrt{\left(\mathrm{C}_{\mathrm{r}}\right)^{*}}
$$

5. Use Equation (7.41), the true raw coincidence rate, $\left(\mathrm{C}_{\mathrm{r}}\right)^{*}$, and its standard deviation, $\left(\sigma_{\mathrm{cr}}\right)^{*}$, to calculate the simulated measured raw coincidence rate, $C_{r}$.

$$
\mathrm{C}_{\mathrm{r}}=\left(\mathrm{C}_{\mathrm{r}}\right)^{*}+\delta_{\mathrm{c}}
$$

where $\delta_{\mathrm{c}}$ is a statistical variant based on the Poisson distribution with a standard deviation, $\left(\sigma_{\mathrm{cr}}\right)^{*}$.

Shift Register Gross R + A Count and Standard Deviation. The gross real plus accidental coincidence count from the shift register is determined from the sum of the real coincidence rate, the accidental coincidence rate, and the count time as defined in the following steps.

1. Use Equations 7.28 through 7.30 to calculate the gross real plus accidental count rate, gross $(r+a)$.

2. Multiply the gross $(r+a)$ by the count time, $t_{c}$, to determine the average gross $R+A$ count.

3. Calculate gross $R+A$ count $=$ average gross $R+A$ count $+\delta_{r+a}$, where $\delta_{r+a}$ is a statistical variant based on the Poisson distribution with a standard deviation equal to $\sqrt{\text { average gross } R+A \text { count }}$.

4. Calculate the standard deviation of the gross real plus accidental count as $\sqrt{\operatorname{gross}(r+a)} * \frac{1}{t_{c}}$.

Shift Register Gross A Count and standard deviation. The shift register gross accidental count and standard deviation are determined from the gross accidental rate and the count time as defined below.

1. Use Equations (7.31) and (7.32) to calculate the gross accidental count rate, gross(Arate).

2. Multiply gross(Arate) by the count time, $t_{c}$, to determine the average gross A count.

3. Calculate gross A count $=$ average A count $+\delta_{a}$, where $\delta_{a}$ is a statistical variant based on the Poisson distribution with a standard deviation equal to $\sqrt{\text { average gross } A \text { count }}$.

4. Calculate the standard deviation of the gross accidental count as $\sqrt{\operatorname{gross}(\text { Arate) }} * \frac{1}{t_{c}}$.

\subsubsection{Simulation of PAN Data Analysis Procedure}

The equations listed below are from the SWEPP Assay System analysis program description report (East, 1997). A computer program was written to implement these calculations for the simulated graphite drum measurements. The program is described in detail in (Meachum, 1997). 
PAN Analysis Procedure Input Parameters. The parameters required for the PAN analysis calculations are listed in Table 7-2. The parameters b2 through b10 are derived using the procedures given in Section 7.2.3, using data from statistical reviews of RTR data, the data base of previous measurements, and shipping records from Rocky Flats Plant. Parameters b11 through b15 are determined from the statistical review of the data base of PAN measurements involving graphite waste forms in drums (See Section 6).

Table 7-2. Parameters required for PAN analysis calculations.

\begin{tabular}{|c|c|}
\hline Parameter & Description \\
\hline b1 & Count time, $t_{c}$ \\
\hline b2 & Raw singles counts, $\left(S_{\mathrm{r}}\right)_{\mathrm{sh}},\left(\mathrm{S}_{\mathrm{r}}\right)_{\mathrm{sy}}$ \\
\hline b3 & Coincidence gate times, $T_{\mathrm{sg}} . \mathrm{T}_{\mathrm{gg}}$ \\
\hline b4 & Number of coincidence gates, $\left(\mathrm{N}_{\mathrm{c}}\right)_{\mathrm{sg}},\left(\mathrm{N}_{\mathrm{c}}\right)_{\mathrm{gg}}$ \\
\hline b5 & Raw coincidence counts, $\left(\mathrm{C}_{\mathrm{r}}\right)_{\mathrm{sg}},\left(\mathrm{C}_{\mathrm{r}}\right)_{\mathrm{lg}}$ \\
\hline b6 & Raw coincidence count standard deviations, $\left(\sigma_{\mathrm{c}}\right)^{*}{ }_{\mathrm{sg}},\left(\sigma_{\mathrm{c}}\right)^{*}{ }_{\mathrm{lg}}$ \\
\hline b7 & Shift register background $\mathrm{R}+\mathrm{A}$ count error \\
\hline b8 & Shift register background A count error \\
\hline b9 & Background coincidence count rate error, $\left(\sigma_{\mathrm{bc}}\right)_{\mathrm{sg}},\left(\sigma_{\mathrm{bc}}\right)_{\mathrm{lg}}$ (for the daily background drum) \\
\hline $\mathrm{b} 10$ & Mass fraction of $\mathrm{Pu}-240, \mathrm{MF}_{40}$ (set equal to 0.0582 ) \\
\hline b11 & Background singles count rate, $\left(B_{\mathrm{s}}\right)_{\mathrm{sh}},\left(\mathrm{B}_{\mathrm{s}}\right)_{\mathrm{sy}}$ (for the daily background drum) \\
\hline b12 & Background coincidence count rate, $\left(\mathrm{B}_{\mathrm{c}}\right)_{\mathrm{sh}},\left(\mathrm{B}_{\mathrm{c}}\right)_{s y}$ (for the daily background drum) \\
\hline b13 & Absorption index, $\mathrm{AI}$ \\
\hline $\mathrm{b} 14$ & Shift register background $\mathrm{R}+\mathrm{A}$ count \\
\hline b15 & Shift register background A count \\
\hline
\end{tabular}

Simulation of the PAN Analysis of the Passive Response Data. The general steps in the PAN analysis simulation are:

1. Using the input parameters from Table 7-2, the Pu mass as determined from the short-gate coincidence data (referred to as the short-gate $\mathrm{Pu}$ mass), the $\mathrm{Pu}$ mass as determined from the long-gate coincidence data (referred to as the long-gate Pu mass) and the Pu mass as determined by the shift register coincidence data (referred to as SRC Pu mass) are calculated separately using exact duplicate algorithms of those found in the current version of the PAN data analysis program.

2. The SRC Pu mass will be used as the selected passive Pu mass unless the shift register mass is questionable or the shift register is not operable. If the SRC Pu mass is not used then the shortgate or long-gate Pu mass will be used. In this situation, the PAN data analysis program selects either the short-gate Pu mass or the long-gate Pu mass based on which of the two Pu masses has the smallest relative error.

As has been the case in determining the simulated PAN response data above, the approach shown below is the same for both the shielded singles/short-gate coincidence data and the system singles/long-gate coincidence data and therefore the subscripts "sh", "sg", "sy", "lg" are implied in the equations shown. Since the shift register is different the analysis of its data will be discussed separately below.

Long-gate and short-gate data analysis. To determine the Pu mass from the long-gate and shortgate coincidence data, the following steps are performed. 
1. Use Equation (7.42) to compute total gate times, $\left(\mathrm{T}_{\mathrm{g}}\right)_{\mathrm{sg}},\left(\mathrm{T}_{\mathrm{g}}\right)_{\mathrm{lg}}$. Each of these gate times is the total time that the respective coincidence gate is opened over the course of the measurement and is determined from the number of coincidence gates, $N_{c}$, and the open time per gate, $T$.

$\mathrm{T}_{\mathrm{g}}=\mathrm{TN}_{\mathrm{c}}$

2. Use Equation (7.43) to compute total live times, $\left(T_{1}\right)_{\mathrm{sg}},\left(\mathrm{T}_{\mathrm{l}}\right)_{\mathrm{lg}}$. Each of these live times is calculated from the total count time, $t_{c}$, the total gate time, $T_{g}$, for the respective coincidence unit and the delay time per gate, $t_{d}$.

$T_{1}=t_{c}-T_{g}-N_{c} t_{d}$

3. Use Equation (7.44) to compute the gross coincidence rates, $\left(C_{g}\right)_{s g},\left(C_{g}\right)_{\mathrm{lg}}$. Each of these coincidence rates is calculated from the respective raw coincidence count, $C_{r}$, the respective singles count rate, $n$, the respective total gate time, $T_{g}$, and the respective live time, $T_{1}$. The result is a coincidence rate which has been corrected for accidental coincidence events.

$\mathrm{C}_{\mathrm{g}}=\frac{\mathrm{C}_{\mathrm{r}}-\mathrm{nT} \mathrm{T}_{\mathrm{g}}}{\mathrm{T}_{1}}$

4. Use Equation (7.45) to compute the measured coincidence rates, $\left(C_{m}\right)_{s g},\left(C_{m}\right)_{g}$. Each of these rates is calculated from the respective gross coincidence rate, $C_{g}$, and the respective background coincidence rate, $B_{c},(b 9)$. The result is the measured coincidence rate which has now been corrected for both accidental coincidence events and background coincidence events.

$C_{m}=C_{g}-B_{c}$

5. Use Equation (7.46), $S_{r}, t_{c}$, and $B_{s}$ to compute the net singles count rates, $\left(S_{m}\right)_{s h},\left(S_{m}\right)_{s y}$.

$S_{m}=\frac{S_{r}}{t_{c}}-B_{s}$

6. Use Equation (7.47), $S_{r}$, and $t_{c}$ to compute the raw singles count rate standard deviations, $\left(\sigma_{n}\right)_{s h}$, $\left(\sigma_{\mathrm{n}}\right)_{\mathrm{sy}}$. As calculated here, these values are based on counting statistics only.

$\sigma_{n}=\frac{1}{t_{c}} \sqrt{S_{r}}$

7. Use Equation (7.48), $T_{l}, C_{r}, \sigma_{n}$, and $T_{g}$ to compute the gross coincidence rate standard deviations, $\left(\sigma_{\mathrm{cg}}\right)_{\mathrm{sg}},\left(\sigma_{\mathrm{cg}}\right)_{\mathrm{lg}}$.

$\sigma_{\mathrm{cg}}=\frac{1}{\mathrm{~T}_{1}} \sqrt{\mathrm{C}_{\mathrm{r}}+\left(\sigma_{\mathrm{n}} \mathrm{T}_{\mathrm{g}}\right)^{2}}$

8. Use Equation (7.49) to compute the measured coincidence rate standard deviations, $\left(\sigma_{\mathrm{cm}}\right)_{\mathrm{sg}}$, $\left(\sigma_{\mathrm{cm}}\right)_{\mathrm{g}}$. 


$$
\sigma_{\mathrm{cm}}=\frac{1}{\mathrm{~T}_{1}} \sqrt{\left(\sigma_{\mathrm{cg}}\right)^{2}+\left(\sigma_{\mathrm{bc}}\right)^{2}}
$$

9. Use Equation (7.50) to calculate the background singles count rate error, $\left(\sigma_{\mathrm{Bs}}\right)_{\mathrm{sh}},\left(\sigma_{\mathrm{Bs}}\right)_{\mathrm{sy}}$.

$$
\sigma_{B_{s}}=\frac{1}{t_{c}} \sqrt{B_{s}}
$$

10. Use Equation (7.51) to calculate the net singles count rate errors, $\left(\sigma_{\mathrm{sm}}\right)_{\mathrm{sh}},\left(\sigma_{\mathrm{sm}}\right)_{\mathrm{sy}}$.

$$
\sigma_{\mathrm{sm}}=\sqrt{\sigma_{\mathrm{n}}^{2}+\sigma_{\mathrm{s}}^{2}}
$$

11. Compute the moderator index, MI. The moderator index is the same for both the short-gate and long-gate coincidence units.

If $\left(\sigma_{\mathrm{sm}}\right)_{\mathrm{sh}} /\left(\mathrm{S}_{\mathrm{m}}\right)>\mathrm{SLDCR}$ relative error, then the moderator index, MI, is set to the default moderator index for a given waste.

Otherwise, if $\left(S_{m}\right)_{s y} \leq 0$, then $M I$ is set to the moderator index minimum value for a given waste. However, if $\left(S_{m}\right)_{s y}>0$, then MI is set to the lowest of 1) the moderator index maximum value or 2) the maximum of the moderator index minimum value or the $\mathrm{MI}$ as calculated in Equation (7.52).

For graphite waste, the following defaults are applicable:

SLDCR relative error $=0.10$

moderator index minimum value $=0.0$

moderator index maximum value $=0.75$

default moderator index value $=0.14$.

$$
\mathrm{MI}=\left[1-4.42 \frac{\left(\mathrm{S}_{\mathrm{m}}\right)_{\mathrm{sh}}}{\left(\mathrm{S}_{\mathrm{m}}\right)_{\mathrm{sy}}}\right][1.04+0.2924 \ln (\mathrm{AI})]
$$

12. Compute the isotopic correction factor, $\mathrm{CF}_{\mathrm{I}}$.

$$
\mathrm{CF}_{\mathrm{I}}=\frac{\left(\mathrm{MF}_{40}\right)_{\mathrm{wgPu}}}{\mathrm{MF}_{40}}
$$

where the default value for $\left(\mathrm{MF}_{40}\right)_{\mathrm{wgPu}}$ is equal to 0.0582 .

13. Use Equation (7.54) to compute the short-gate coincidence correction factor, $\mathrm{CF}_{\mathrm{sg}}$. 


$$
\mathrm{CF}_{\mathrm{sg}}=\left(\frac{0.8092}{1-\mathrm{MI}}+0.2337\right)^{2}
$$

14. Use Equation (7.55) to compute the long-gate coincidence correction factor, $\mathrm{CF}_{\mathrm{lg}}$.

$$
\mathrm{CF}_{\mathrm{lg}}=\left(\frac{0.5967}{1-\mathrm{MI}}+0.4187\right)^{2}
$$

15. Use Equation (7.56) to compute the short-gate Pu mass, $\left(\mathrm{M}_{\mathrm{Pu}}\right)_{\mathrm{sg}}$.

$$
\left(\mathrm{M}_{\mathrm{Pu}}\right)_{\mathrm{sg}}=(38.6) \mathrm{CF}_{\mathrm{sg}} \mathrm{CF}_{\mathrm{I}}\left(\mathrm{C}_{\mathrm{m}}\right)_{\mathrm{sg}}
$$

16. Use Equation (7.57) to compute the long-gate Pu mass, $\left(\mathrm{M}_{\mathrm{Pu}}\right)_{1 \mathrm{~g}}$.

$$
\left(\mathrm{M}_{\mathrm{Pu}}\right)_{\mathrm{lg}}=(1.85) \mathrm{CF}_{\mathrm{lg}} \mathrm{CF}_{\mathrm{l}}\left(\mathrm{C}_{\mathrm{m}}\right)_{\mathrm{lg}}
$$

17. Use standard error propagation techniques to compute the standard deviation in the short-gate $\mathrm{Pu}$ mass, $\left(\sigma_{\mathrm{Pu}}\right)_{\mathrm{sg}}$, and the long-gate $\mathrm{Pu}$ mass, $\left(\sigma_{\mathrm{Pu}}\right)_{\mathrm{sg}}$ (East, 1997).

18. Select the passive plutonium mass. (For those cases where the shift register data are not available.)

If the absolute value of the relative error for the short-gate coincidence rate is less than the absolute value of the relative error for the long-gate coincidence rate, i.e.

$$
\left|\frac{\left(\sigma_{\mathrm{cm}}\right)_{\mathrm{sg}}}{\left(\mathrm{C}_{\mathrm{m}}\right)_{\mathrm{sg}}}\right| \leq\left|\frac{\left(\sigma_{\mathrm{cm}}\right)_{\mathrm{lg}}}{\left(\mathrm{C}_{\mathrm{m}}\right)_{\mathrm{lg}}}\right|,
$$

and the short-gate coincidence rate is greater than zero, the passive plutonium mass and its error are set equal to the plutonium mass and its error from the short-gate coincidence unit:

$\mathrm{M}_{\mathrm{pu}}=\left(\mathrm{M}_{\mathrm{pu}}\right)_{\mathrm{sg}} \quad$ and $\quad \sigma_{\mathrm{Pu}}=\left(\sigma_{\mathrm{Pu}}\right)_{\mathrm{sg}}$.

Otherwise, the passive plutonium mass and its error are set equal to the plutonium mass and its error from the long-gate coincidence system:

$$
\mathrm{M}_{\mathrm{pu}}=\left(\mathrm{M}_{\mathrm{pu}}\right)_{\mathrm{gg}} \quad \text { and } \quad \sigma_{\mathrm{Pu}}=\left(\sigma_{\mathrm{Pu}}\right)_{\mathrm{gg}} \text {. }
$$

Shift Register Data Analysis. To determine the PU mass from the shift register data, the following calculations are required.

1. Gross $\mathrm{R}+\mathrm{A}$ rate $=\mathrm{Gross} \mathrm{R}+\mathrm{A}$ count $/ \mathrm{t}_{\mathrm{c}}$

2. Gross $A$ rate $=$ Gross $A$ count $/ t_{c}$ 
3. Net $R+A$ rate $=$ Gross $R+A$ count rate - Background $R+A$ count rate

4. Net $\mathrm{A}$ rate $=$ Gross $\mathrm{A}$ count rate - Background $\mathrm{A}$ count rate

5. Net $R$ rate $=$ Net $R+A$ count rate - Net $A$ count rate

6. Corr Net $R$ rate $=($ Net $R$ rate $) \exp \left(\delta_{c} n\right)$

7. $\left(\mathrm{M}_{\mathrm{pu}}\right)_{\mathrm{src}}=(1.85)(0.957)($ Corr Net $\mathrm{R}$ rate $) \mathrm{CF}_{\mathrm{lg}} \mathrm{CF}_{\mathrm{I}}$

The shift register passive plutonium mass is the value presented in the current version of the PAN assay report. These values are compared against the known plutonium mass to assess the total uncertainty of the PAN assay system. Given the plutonium mass (passive) such quantities as total alpha activity, alpha concentration, fissile gram equivalent and thermal power can be derived. The uncertainties for derived quantities can then be propagated using the total uncertainty of the plutonium mass value determined from this method combined with uncertainties assigned to the mass fractions and other parameters. If the SWEPP gamma spectrometer mass ratio values are being used to obtain the derived quantities then the uncertainties associated with the mass ratios would be included in the propagation of the total uncertainties in the derived quantities. 


\section{SURROGATE WASTE DRUM BENCHMARK RESULTS}

To validate the computer modeling process used for simulating the PAN system results, simulations of actual measurements on a surrogate graphite waste drum were performed. By comparing the actual to simulated measurement results for the controlled surrogate drum configurations, the ability of the simulation to approximate the PAN system performance can be evaluated. The primary analysis of concern is the comparison of the final Pu gram quantities reported by the actual and simulated PAN measurements. Various statistical significance tests are utilized in the comparisons. Exact p-values are generally given for each test. References in the discussion to effects being statistically significant refer to cases with p-values less than 05 .

Matrix configuration and related measurement parameters for the surrogate drum simulations followed the same general approach as that used for the waste drum simulations. A few minor changes were made to accommodate the different assumptions that can reasonably be made about surrogate vs. waste drums and the different types of data that are available. One such difference, described in detail in Section 6, involved the distributions used to generate the absorber index values. Another difference occurred in the generation of background count values. For waste drum simulations, it is necessary to generate background counts for both the waste drum measurement and its associated daily background drum measurement. In the simulations of the surrogate drum runs however, it is only necessary to simulate the background counts associated with the surrogate drum measurement since the actual background counts for the daily background drum measurements can be used. Using the actual values for the daily background drum measurements allows for a more accurate assessment of the ability of the simulation process to mimic the PAN system performance by removing a source of variability extraneous to the assessment of the PAN simulation (i.e., variability in the daily background drum values).

To facilitate more accurate comparisons of the simulated and actual PAN measurements for the purpose of adjusting the simulation output, the surrogate drum measurements were first simulated assuming an infinite count time. This eliminates another source of variability (i.e., counting statistics error) from affecting an assessment of the magnitude of the adjustment required. Following the assessment and adjustment for simulation bias, the simulations were rerun with the count time set to 600 seconds (the actual count time in the surrogate drum runs) to make an assessment of the ability of the simulation process to mimic the variability in the actual PAN measurements.

\subsection{Description of the Surrogate Drum and Configuration Variations}

The surrogate graphite waste drum is specified in detail by Becker in an INEEL internal document (Becker, 1995). The drum consists of a standard 207 liter waste drum with a 90 mil polyethylene liner inside of which is an internal structure which simulates a nominal graphite content matrix. The major components are graphite bricks, an internal support structure, and source positioning wands.

The bulk of the matrix is composed of bricks of graphite with holes and inter-spacings such that the average density is equal to the nominal density, $0.7959 \mathrm{~g} / \mathrm{cm}^{3}$. Seven layers of these bricks are included such that the fill height is equal to the nominal fill height, $47 \mathrm{~cm}$. Carbon pins along with nylon ties are used to hold carbon bricks in position. Graphite bars are used between the layers to provide spacing and are sized to achieve the density mentioned above. The whole brick assembly is clamped together by the aluminum support structure.

Three source positioning tubes are included. Source tube \#1 is located at the center of the drum, source tube \#2 is located halfway between the drum center and the edge of the graphite structure and source tube 
\#3 is located at the edge of and external to the graphite structure. In each tube there is a source wand. One wand design accommodates 11 one gram, one inch diameter plutonium disk sources (referred to as NAD sources). The second wand design accommodates 10 thirty gram ZPPR fuel plates. The source wand orientation in the source tube is indexed to provide angular orientation with respect to the drum in $45^{\circ}$ increments. The drum itself is indexed also in $22.5^{\circ}$ increments of the drum relative to the detector enclosure.

The cases identified in the surrogate drum series of measurements vary the number and location of one gram NAD sources, the angular orientation of the sources within the drum and the orientation of the drum within the enclosure. The matrix composition and fill are fixed throughout the measurements.

Fifteen different runs of the graphite surrogate drum were simulated using the methods described above. Because of their importance to evaluating the simulation process, five of these runs were zero source runs. One of the zero source runs produced an atypical (outlier) result, perhaps due to a chance cosmic event, and was not included in the analysis, leaving a total of 14 runs for this analysis. Table 8-1 describes the various source configurations used in the 9 runs for which sources were included. For all configurations only the angular orientation of the drum in the assay chamber, the number of Pu NAD sources, and their positions were varied. Matrix parameters were not varied.

Table 8-1. Surrogate drum configurations for which simulations were performed (runs with sources present only).

\begin{tabular}{|c|c|c|c|c|c|}
\hline \multirow[b]{2}{*}{$\begin{array}{l}\text { Surrogate drum } \\
\text { run number }\end{array}$} & \multirow[b]{2}{*}{$\begin{array}{l}\text { Position of drum } \\
\text { in PAN chamber }\end{array}$} & \multicolumn{3}{|c|}{ Positions containing NADs } & \multirow[b]{2}{*}{$\begin{array}{c}\text { Total } \mathrm{Pu} \\
\text { mass }\end{array}$} \\
\hline & & Tube 1 & Tube 2 & Tube 3 & \\
\hline grap01.001 & left & $5-11$ & & & 7.168 \\
\hline grap05.001 & left & 6,10 & & & 2.048 \\
\hline grap12.001 & right & & & $5-7$ & 3.072 \\
\hline grap 25.001 & door & & $9-11$ & & 3.072 \\
\hline grap27.001 & back & & $5-7$ & & 3.072 \\
\hline grap34.001 & rotate & $9-11$ & $9-11$ & $9-11$ & 9.216 \\
\hline grap36.001 & back & $6,8,10$ & $6,8,10$ & $6,8,10$ & 9.216 \\
\hline grap42.001 & right & $5-7$ & $5-7$ & $5-7$ & 9.216 \\
\hline grap48.001 & door & $7-8$ & $5-6$ & $9-10$ & 6.144 \\
\hline
\end{tabular}

\subsection{Bias Adjustment}

The initial simulation results for the surrogate graphite waste drum measurements showed a high degree of correlation with the actual PAN system results. The Pearson's $r$ correlation coefficient for the simulated vs. actual PAN measured mass values was .994 (based on the infinite count time simulations). However, a regression analysis of the data indicated some bias was present in the simulations. Regressing the actual PAN measurements on the simulated results produced the following equation:

actual PAN measured mass $=0.108+0.890($ simulated PAN measured mass $)$.

The intercept from the regression equation represents the amount of constant bias in the simulation process while the slope is indicative of relative bias. (If there were no bias, the intercept would be zero, and the slope 1.0.) To compensate for these bias relationships, the above regression equations were applied to the raw output from the PAN simulations (for both the surrogate and waste drum simulations) 
to produce adjusted results. All analyses in the remainder of this report are based on these adjusted values.

\subsection{Simulated vs. Actual PAN System Reported Pu Quantities}

Table 8-2 compares the simulated and actual PAN system calculated Pu quantities for each of the graphite surrogate drum simulations. The true Pu mass in the drum is also given for reference purposes. (Note, because this section focuses on assessing bias in the simulation process, the infinite count time simulation results are used.)

The actual vs. simulated results are also plotted in Figure 8-1. The line in the graph indicates where the data would fall if there were perfect agreement of the simulated and actual PAN values. Note that because of the bias adjustment described above, the values should fall about this line on average. However the adjustment does not guarantee that the agreement will be equally good over all ranges of configurations, nor that the range of deviations from the line will be small. (For example, there could be large positive bias for low gram quantities but large negative bias for high gram quantities. In that case, while the average bias would be zero, the agreement for particular values would be very poor.)

That the data in Figure 8-1 all fall closely about the line of perfect agreement indicates that the simulation modeling process works well over the entire range of $\mathrm{Pu}$ gram quantities considered. The degree of agreement can be further quantified by considering a regression of the actual PAN values on the simulated PAN values. If the simulation process is equally accurate at all gram quantity values, the regression line should approximate the line of perfect agreement which has an intercept value of 0.0 and a slope of 1.0 , i.e., the regression equation should approximate the simple equation $y=x$. Furthermore, the regression should indicate a high correlation between the two values.

Regressing the actual PAN values on the simulated values produced a regression equation of $y=0.0004$ $+.9997^{*} \mathrm{x}$. The standard error for the intercept was .18, indicating it is not significantly different from $0.0(\mathrm{p}=1.00)$. The standard error for the slope was .032 , indicating it is not significantly different from $1.0(\mathrm{p}=.99)$. Thus there is no evidence of a statistically significant deviation from the line of perfect agreement. As with the data prior to adjustment, a correlation value of $r=.994$ was obtained from the regression, indicating a close degree of agreement for the two sets of data.

\subsection{Comparison of Uncertainty Results}

While the previous sections compared simulated to actual PAN measurement results, this section compares simulated and actual PAN measurements to the true Pu quantities known to be in each drum. This provides a comparison of the PAN system uncertainty as estimated by the simulations vs. that obtained from actual drum assays. Because this section focuses on the PAN simulation routines ability to match the overall uncertainty of the actual PAN measurements, including the variability, the simulation data for the set count time of 600 seconds are used.

Figure 8-2 compares plots of actual and simulated PAN measurements vs. the true Pu quantity in the surrogate drum. The plots show that the simulated data mimic the actual PAN data quite closely in terms of their relationship to the true Pu quantities in the surrogate drum. The simulation data compare favorably both in terms of the general trend in relation to the line of perfect agreement (which relates to bias) and the variability among repeat measurements at various Pu quantities (which relates to precision). 
Table 8-2. Comparison of simulated vs. actual PAN measurement results for the 14 surrogate graphite waste drum configurations (infinite count time data).

\begin{tabular}{|c|c|c|c|c|}
\hline $\begin{array}{c}\text { Surrogate drum } \\
\text { run number }\end{array}$ & $\begin{array}{c}\text { True Pu quantity in } \\
\text { drum (grams) }\end{array}$ & $\begin{array}{c}\text { Actual PAN shift } \\
\text { register measurement } \\
\text { result (grams) }\end{array}$ & $\begin{array}{c}\text { Simulated PAN } \\
\text { measurement result } \\
\text { (grams) }\end{array}$ & $\begin{array}{c}\text { Relative \% error, } \\
\text { simulation vs. actual } \\
\text { PAN result }\end{array}$ \\
\hline grap01.001 & 7.168 & 7.870 & 8.361 & 6.2 \\
\hline grap05.001 & 2.048 & 2.375 & 2.784 & 17.2 \\
\hline grap12.001 & 3.072 & 4.447 & 4.375 & -1.6 \\
\hline grap25.001 & 3.072 & 3.947 & 3.730 & -5.5 \\
\hline grap27.001 & 3.072 & 3.642 & 4.074 & 11.9 \\
\hline grap34.001 & 9.216 & 8.898 & 8.687 & -2.4 \\
\hline grap36.001 & 9.216 & 9.087 & 9.865 & 8.6 \\
\hline grap42.001 & 9.216 & 11.585 & 10.558 & -8.9 \\
\hline grap48.001 & 6.144 & 6.867 & 6.756 & -1.6 \\
\hline grapze.001 & 0.0 & 0.012 & 0.107 & 785.3 \\
\hline grapze.002 & 0.0 & 0.374 & 0.107 & -71.3 \\
\hline grapze.003 & 0.0 & 0.044 & 0.107 & 142.5 \\
\hline grapze.004 & 0.0 & 0.386 & 0.107 & -72.2 \\
\hline grapze.005 & 0.0 & 0.185 & 0.107 & -42.0 \\
\hline
\end{tabular}

The bias and precision components of the simulated and real PAN data are more easily evaluated by considering the difference between the PAN results and the true Pu quantities. These data are shown in Figure 8-3. Absolute rather than relative errors are plotted in the figure because the absolute errors are more constant. As will be done for the full uncertainty analysis, bias and precision can be assessed by considering the fitted regression lines to the data in these plots. If there were no bias in the PAN system, all the difference data would average to zero. Hence, to the extent that the regression line deviates from a horizontal line through zero on the plot, bias is indicated. A regression intercept other than zero is indicative of a constant bias in the PAN system. A significant slope indicates a relative bias component. The extent of precision error is indicated by the variability of the absolute difference values about the regression line.

The regression lines in Figure 8-3 suggest that the simulated data are exhibiting somewhat more bias than the actual PAN measurements. However, neither the intercepts nor the slopes for the regression of the actual PAN data differ significantly from the corresponding coefficient in the regression for the simulated data. Also, for both the simulated and actual PAN measurements, neither the intercepts nor slopes are significantly different from zero. Thus the regression analyses of both the actual and simulated data suggest that there is no bias in the PAN measurements for the graphite surrogate waste drum measurements. (The conclusion of no bias is somewhat contradicted however by the fact that all but two of the 14 surrogate drum measurements produced a measured Pu mass value greater than the actual Pu mass placed in the drum.)

The spread of the data about the regression lines also appears similar in the simulated vs. actual PAN data plots, indicating comparable precision estimates as well. A more precise comparison of the precision in each case can be obtained by considering the square root of the mean square error from each regression, often called the standard error of estimate. The mean square error is an estimate of the variance of the data about the regression line and hence its square root is a precision estimate. For the actual PAN data this value is .69 while for the simulated PAN data it is .58 . While these numbers suggest that the simulation variability is slightly less than that for the actual PAN data, the difference is not statistically significant $(p=.29)$, so it is reasonable to assume the simulation variability is representative of that for the actual data. (Note that the mean square error in a standard least squares 
regression model assumes a constant variance for all mass values when in actuality the PAN variance may increase with mass. This variance relationship is considered in detail in the waste drum simulation data, but for purposes of assessing the adequacy of the simulation process, the simplified equal variance model is sufficient.)

The surrogate drum simulation data also indicate that the agreement of the simulated values with the actual measured values is much better than the agreement of the actual measured values with the corresponding true Pu quantity in the drum. Thus any deviations of the simulated values from the actual measured values are likely to be small relative to the variability of the PAN system itself to changes in factors such as drum configuration.

Note that all the values reported above measure the uncertainty for the PAN system only for the surrogate drum configurations and for a limited range of mass values. Hence they should not be interpreted as indicative of the overall performance of the PAN system on graphite waste drums. Overall performance should be based on the results for the simulated waste drum sample reported in the next section.

It should also be noted that in the actual PAN calculations, and therefore in the simulation of the PAN calculations, the isotopic correction factor was taken to be 1.0. This implies that the mass fraction of Pu240 is 0.0582 (i.e., this is the nominal value assumed in the PAN calculations for weapons grade plutonium). The actual Pu-240 mass fraction for the NADs is .0528. Hence for surrogate runs involving NAD sources, this value was used in simulating the PAN response in terms of singles and coincidence counts. If isotopic correction factors of $0.0582 / 0.0528=1.10$ for surrogate runs using NADs had been used in the PAN analysis, the difference between the actual and PAN reported mass values (actual or simulated) might be much closer. However, it is still appropriate to use an isotopic correction factor of 1.0 in the PAN calculations because that is the assumed (default) value in the actual operation of the PAN system, unless gamma spectrometric data indicates conclusively to the contrary. 
Figure 8-1. Comparison of simulated vs. actual PAN system mass values for surrogate graphite waste drums (infinite count time data)

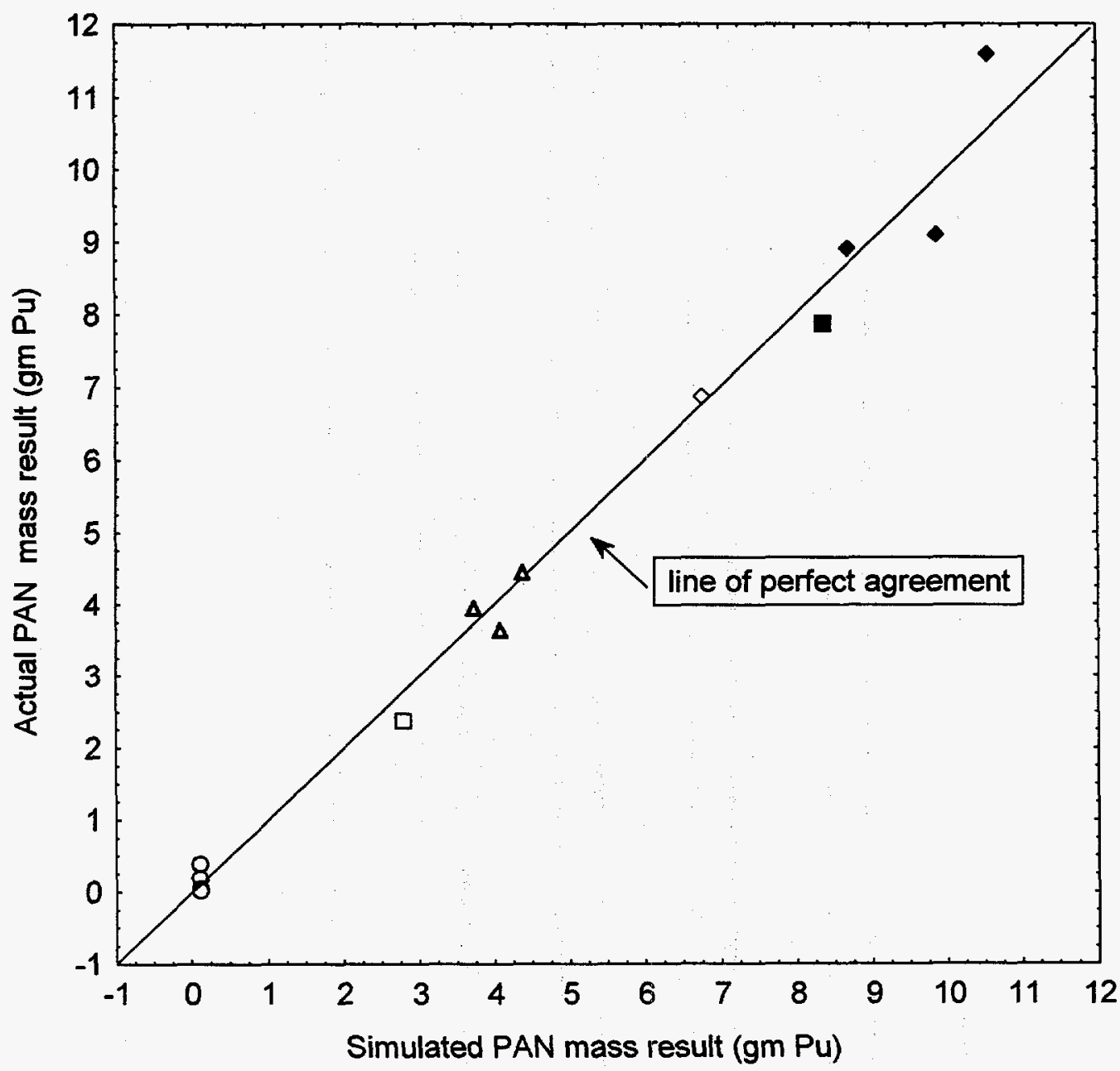

True Pu mass in drum (g)

a 0.0

2.048

$\triangle \quad 3.072$

$\diamond \quad 6.144$

- 7.168

- 9.216

Simulated PAN mass result ( $\mathrm{gm} \mathrm{Pu}$ ) 
Figure 8-2. Actual and simulated PAN results vs. true Pu quantity (600 sec. count time data)
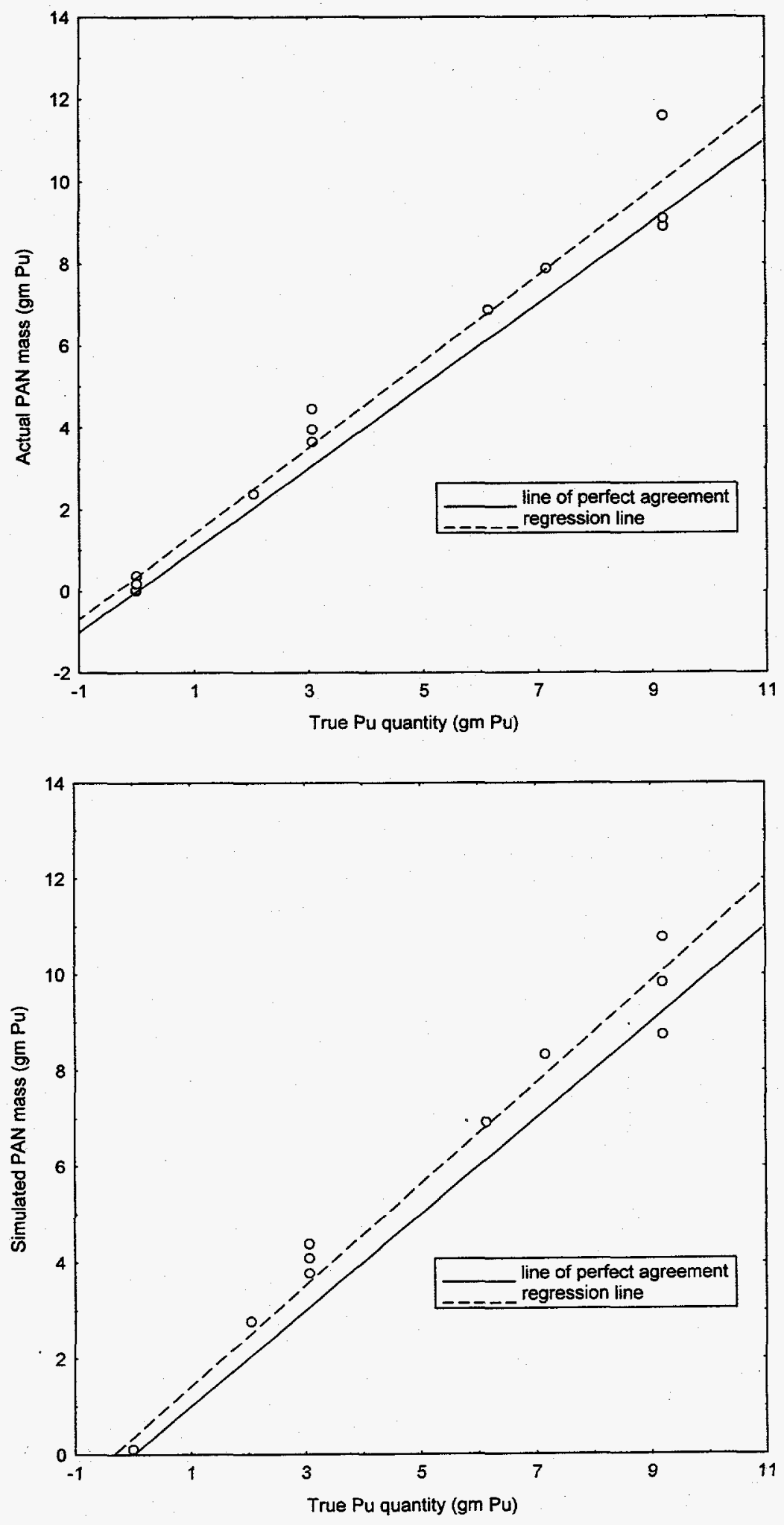
Figure 8-3. Comparison of deviations from true Pu quantities (600 sec. count time data)
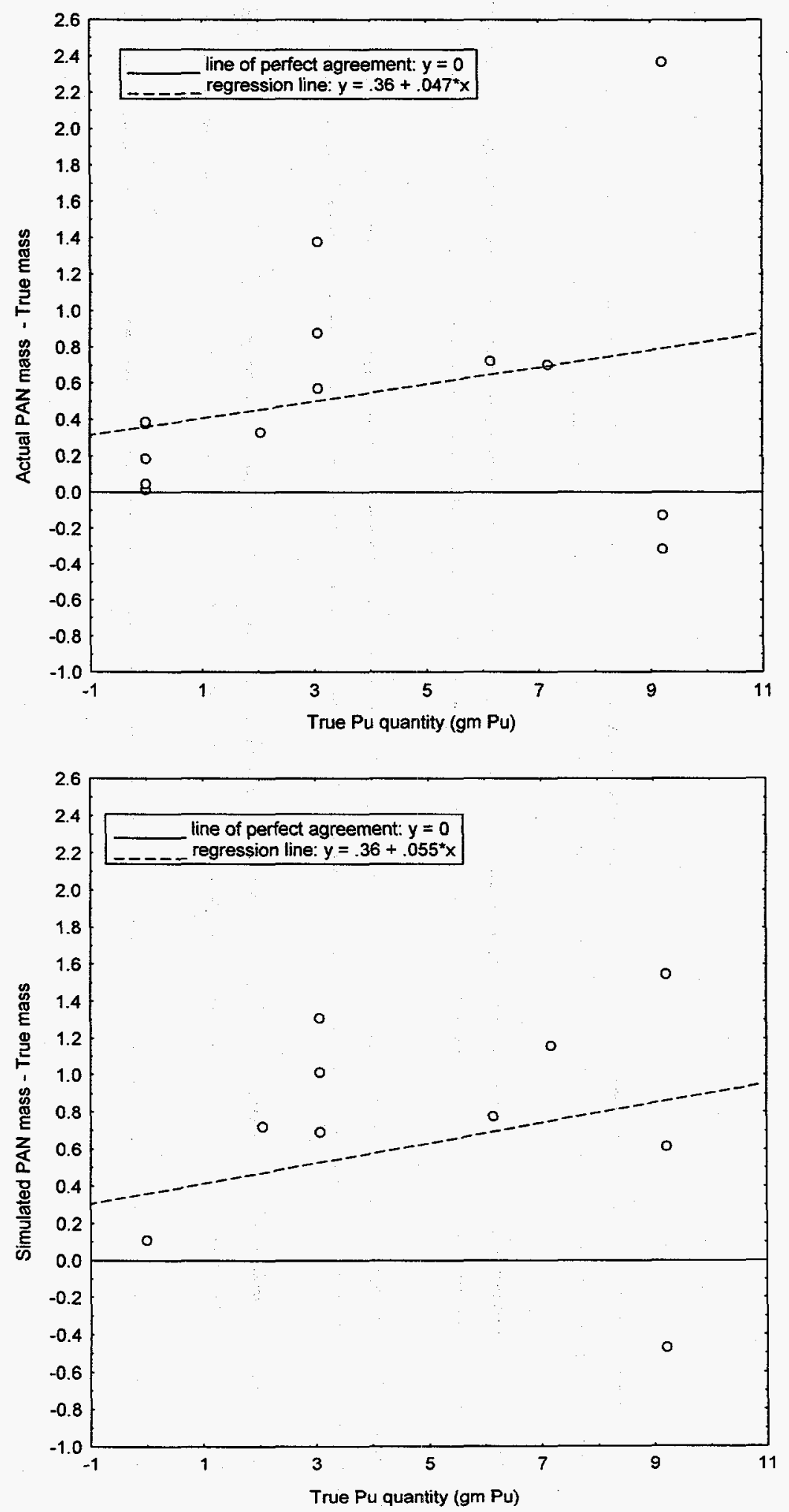


\section{UNCERTAINTY ANALYSIS USING THE LATIN HYPERCUBE SAMPLE SIMULATED WASTE RESULTS}

Two results were produced for each simulated waste drum. The first result was that using an infinite count time. These results give the best estimate of the actual expected PAN performance for that drum's configuration. The second result uses a count time of $1200 \mathrm{sec}$., the same as for actual waste drum measurements. Since the 1200 second count time data contains additional random error components, they are not as accurate as the infinite count time data for calculating expected PAN performance (i.e., bias). However, the 1200 second count time data provide better estimates of the expected variability (precision). The infinite count time data are used for all the analyses described below, except for that dealing with precision estimates.

The simulated PAN measured Pu mass values for the infinite count time data are given in Table 9-1. The uncertainty in each result is obtained by comparison to the Pu quantity specified for each drum in Table 9-1. (For brevity, the Pu quantity specified for each simulated drum will generally be referred to in this section as the "actual" or "true" quantity, and the simulated PAN system measurement result as simply the "PAN Pu" quantity. Also, the PAN Pu quantities have been adjusted for the bias discussed in Section 8 for the surrogate drums.)

The true vs. PAN measured Pu quantities are plotted in Figure 9-1. As in previous figures, the line of perfect agreement specified in the plot indicates where the data would fall if there were no measurement bias or precision error. That the data fall nearly on the line of perfect agreement indicates essentially no bias in the PAN measurements for graphite waste. The "tightness" of the scatter of points about the regression line is an indicator of the degree of precision in the measurements.

Bias and precision in the results can be quantified by considering the following basic mathematical model for the data in Figure 9-1

$y_{i}=\alpha+\beta\left(x_{i}\right)+\varepsilon_{i}$

where $y_{i}$ is the PAN system Pu measurement for drum $i$ and $x_{i}$ is the true Pu quantity in the drum. The parameters $\alpha$ and $\beta$ relate to the bias in the measurement process. If $\alpha \neq 0$ and $\beta=1$, then there is a constant bias in the measurement system. If $\alpha=0$ and $\beta \neq 1$, then there is constant relative bias in the system. It is also possible to have both constant and relative bias terms at the same time. The term $\varepsilon_{\mathrm{i}}$ in the model is the random error component of the measurement. The random errors are assumed to have a mean of zero. Precision of the measurement system is evaluated by considering the variance (or equivalently the standard deviation) of the $\varepsilon_{\mathrm{i}}$ values.

Using this basic measurement model for the PAN system, bias and precision can be evaluated by applying appropriate statistical analysis to the data from the simulations. These calculations are described in detail below.

\subsection{Bias}

Simply defined, bias is the expected difference between the measured and actual Pu quantities. Thus it can be assessed more directly by subtracting the true Pu quantity $x_{\mathrm{i}}$ from both sides of Equation 9.1 to give: 
Table 9-1. Simulated PAN system measurement results for the Latin hypercube sample (infinite count time data).

\begin{tabular}{|c|c|c|c|}
\hline Drum number & $\begin{array}{l}\text { True Pu mass } \\
\text { (grams) }\end{array}$ & $\begin{array}{l}\text { PAN Pu Mass } \\
\quad(\text { grams })\end{array}$ & Relative \% error \\
\hline 1 & 18.80 & 18.59 & -1.11 \\
\hline 2 & 3.48 & 3.42 & -1.83 \\
\hline 3 & 79.90 & 83.57 & 4.59 \\
\hline 4 & 34.70 & 31.45 & -9.37 \\
\hline 5 & 27.40 & 25.04 & -8.62 \\
\hline 6 & 8.49 & 8.41 & -.98 \\
\hline 7 & 16.20 & 16.85 & 4.01 \\
\hline 8 & 14.70 & 13.84 & -5.87 \\
\hline 9 & 20.90 & 20.00 & -4.29 \\
\hline 10 & 11.30 & 9.17 & -18.83 \\
\hline 11 & 15.40 & 14.10 & -8.42 \\
\hline 12 & 13.30 & 13.10 & -1.48 \\
\hline 13 & 29.50 & 31.02 & 5.14 \\
\hline 14 & 21.70 & 21.71 & .06 \\
\hline 15 & 3.08 & 2.99 & -2.95 \\
\hline 16 & 14.10 & 14.02 & -.60 \\
\hline 17 & 20.10 & 22.24 & 10.64 \\
\hline 18 & 34.90 & 32.51 & -6.84 \\
\hline 19 & 32.30 & 33.64 & 4.15 \\
\hline 20 & 10.10 & 9.93 & -1.70 \\
\hline 21 & 11.50 & 11.26 & -2.11 \\
\hline 22 & 11.00 & 10.61 & -3.59 \\
\hline 23 & 43.00 & 45.31 & 5.37 \\
\hline 24 & 25.40 & 25.13 & -1.05 \\
\hline 25 & 19.10 & 18.81 & -1.51 \\
\hline 26 & 51.30 & 55.93 & 9.03 \\
\hline 27 & 34.20 & 33.57 & -1.83 \\
\hline 28 & 24.70 & 24.13 & -2.29 \\
\hline 29 & 10.40 & 9.08 & -12.71 \\
\hline 30 & 6.45 & 5.65 & -12.33 \\
\hline 31 & 7.46 & 8.93 & 19.66 \\
\hline 32 & 7.58 & 8.83 & 16.49 \\
\hline 33 & 1.18 & 1.13 & -4.34 \\
\hline 34 & 9.95 & 9.00 & -9.57 \\
\hline 35 & 5.41 & 5.64 & 4.18 \\
\hline 36 & 24.10 & 23.93 & -.72 \\
\hline 37 & 22.00 & 21.20 & -3.64 \\
\hline 38 & 4.74 & 5.89 & 24.18 \\
\hline 39 & 15.00 & 15.47 & 3.11 \\
\hline 40 & 9.27 & 8.07 & -12.92 \\
\hline 41 & 10.80 & 10.46 & -3.13 \\
\hline 42 & 41.30 & 43.96 & 6.43 \\
\hline 43 & 14.00 & 12.22 & -12.74 \\
\hline 44 & 17.00 & 19.00 & 11.75 \\
\hline 45 & 11.70 & 12.52 & 7.04 \\
\hline 46 & 7.98 & 7.61 & -4.67 \\
\hline 47 & 6.57 & 5.86 & -10.87 \\
\hline 48 & 16.40 & 16.76 & 2.19 \\
\hline 49 & 11.90 & 10.37 & -12.89 \\
\hline 50 & 12.90 & 13.22 & 2.49 \\
\hline
\end{tabular}




$$
\begin{aligned}
y_{i}-x_{i} & =\alpha+\beta\left(x_{i}\right)-x_{i}+\varepsilon_{i} \\
& =\alpha+\beta^{*}\left(x_{i}\right)+\varepsilon_{i}
\end{aligned}
$$

where $\beta^{*}=\beta-1$. The meanings of $\alpha$ and $\varepsilon_{i}$ remain the same as before. $\beta^{*}$ still relates to relative bias, but now no relative bias results in a value of 0 for $\beta^{*}$. Based on this equation then, the bias in a PAN measurement of $x$ grams $\mathrm{Pu}$ is simply $\alpha+\beta^{*}(\mathrm{x})$, the expected value of Equation 9.2. Since the parameters $\alpha$ and $\beta^{*}$ are not known they must be estimated using regression techniques applied to the Latin hypercube data as described below.

The data for this revised model are plotted in Figure 9-2. If there were no bias in the measurements, the data in Figure 9-2 would be centered around the line of perfect agreement. (Variability about the line would represent the random error or precision of the measurements.) That most of the data points fall below this line indicates once again a negative bias in the PAN measurement system.

A regression analysis was performed to estimate the parameters $\alpha$ and $\beta^{*}$ in Equation 9.2. Since the variability of the data increases with increasing true $\mathrm{Pu}$ quantity, a weighted least squares analysis was performed (Neter and Wasserman, 1974). Ideally, weights should be $1 / \sigma_{x}{ }^{2}$ where $\sigma_{x}{ }^{2}$ is the variance of $\varepsilon$ for a given value of $x$. These values are not known, so both the variances and the regression coefficients were estimated by the following iterative method.

\subsubsection{Weighted least squares estimation}

In the first iteration of the weighted least squares analysis the weights were assumed to all be 1.0 (i.e., an ordinary least squares analysis was performed). Substituting these values into Equation 9.2, estimated values for $\varepsilon_{i}$ were obtained for each of the $x_{i}$ data points. In the case of Equation 9.2 , the standard deviation of the $\varepsilon_{i}$ are expected to increase as $x_{i}$ increases. A numerical estimate of this increasing relationship was obtained by regressing the absolute values of the $\varepsilon_{\mathrm{i}}$ on $\mathrm{x}_{\mathrm{i}}$. (The absolute value of $\varepsilon_{\mathrm{i}}$ is an estimate of its standard deviation.) The estimated standard deviations from this regression were then squared and, after taking reciprocals, used as weights in the second iteration of the weighted least squares analysis. This yielded new estimates of $\alpha$ and $\beta^{*}$, from which new estimates of the $\varepsilon_{\mathrm{i}}$ could be obtained. This iterative process was repeated until the estimates of $\alpha$ and $\beta^{*}$ did not change in the first two significant digits.

\subsubsection{Weighted Least Squares Analysis Results}

The weighted least squares analysis produced an estimated value of -.176 for $\alpha$ (with standard error .112) and -.0073 for $\beta^{*}$ (with standard error .0160 ). Both estimates were not significantly different from zero ( $p=.88$ for $\alpha$ and $p=.65$ for $\beta^{*}$ ). Furthermore, models including only the $\alpha$ or $\beta^{*}$ terms also indicated a lack of bias. Therefore, for graphite waste, the PAN system can be considered to produce unbiased measurements. That is, the model for bias is simply

$\mathrm{y}_{\mathrm{i}}-\mathrm{x}_{\mathrm{i}}=\varepsilon_{\mathrm{i}}$

and since the $\varepsilon_{\mathrm{i}}$ have mean zero, the expected bias is zero.

The uncertainty associated with the model of zero bias can be estimated from the data. For a given value of $x$ (i.e., the true Pu quantity), the bias estimate is zero and its uncertainty, expressed as a standard deviation, is 


$$
s_{b_{x}}=\frac{s_{m_{x}}}{\sqrt{n}}
$$

where the term in the numerator is the estimated matrix precision error for $\mathrm{x}$ as derived in Section 9.2 below, and $n$ is the number of samples comprising the data set ( $=50$ for the graphite data analysis).

\subsection{Matrix Precision Error}

Since the data analyzed above was from the infinite count time simulations, there is no counting statistics error, so all the observed variability is due to matrix effects. We refer to this as matrix precision error. Total precision error is the combination of matrix precision error and counting statistics error. The matrix precision error is assessed by evaluating the variance of the $\varepsilon_{\mathrm{i}}$ values in Equation 9.2. Because we have found that both $\alpha$ and $\beta^{*}$ are essentially zero, the estimated $\varepsilon_{\mathrm{i}}$ values are simply the differences between the PAN and true Pu quantities, as stated in Equation 9.3.

By considering a regression of the absolute values of the estimated $\varepsilon_{\mathrm{i}}$ values on the true $\mathrm{Pu}$ masses (in the same way as was done in the weighted least squares analysis) a relationship for the matrix precision error can be derived. The data for the regression are plotted in Figure 9-3.

In the regression analysis of the matrix precision error data, the intercept was found to be non-significant $(p=.43)$ and was set to zero. The final regression model for the matrix precision error is

$$
\mathrm{s}_{\mathrm{m}_{\mathrm{x}}}=.055 * \mathrm{x}
$$

where $\mathrm{x}$ is the true Pu quantity in the drum and the matrix precision error is expressed as a standard deviation. Thus matrix variability from drum to drum induces a 5.5\% standard deviation in the measured PAN responses for graphite waste. The standard error on the .050 regression parameter is .005 , so the true matrix precision error may be as little as $4.5 \%$ or as high as $5.5 \%$ (95\% confidence bounds).

\subsection{Total Precision Error}

Total precision error is comprised of the matrix precision error and the counting statistics error. Expressed as a standard deviation, it is estimated by calculating the square root of the sum of the squared counting statistics and matrix precision standard deviation values. Combining matrix precision error as calculated using Equation 9.5 with the counting statistics errors for the simulated graphite waste drums produced the data in Figure 9-4. (Note here the 1200 second count time data are used to obtain the counting statistics error rather than the infinite count time data.). The regression equation for these data yields the following formula summarizing total precision error for graphite drums:

$\mathrm{s}_{\mathrm{t}_{\mathrm{x}}}=.25+.061 * \mathrm{x}$

where $\mathrm{x}$ is the true $\mathrm{Pu}$ quantity in the drum and the total precision error is expressed as a standard deviation. This formula represents the overall trend in total precision error across all graphite drums but should not be used to estimate total precision error for a specific single drum. The best estimate for a single drum is simply that drum's counting statistics error combined in quadrature with the matrix precision error as calculated by Equation 9.5 . 
Figure 9-1. PAN Pu measurements vs. true Pu quantity for the simulated graphite waste drums (infinite count time data)

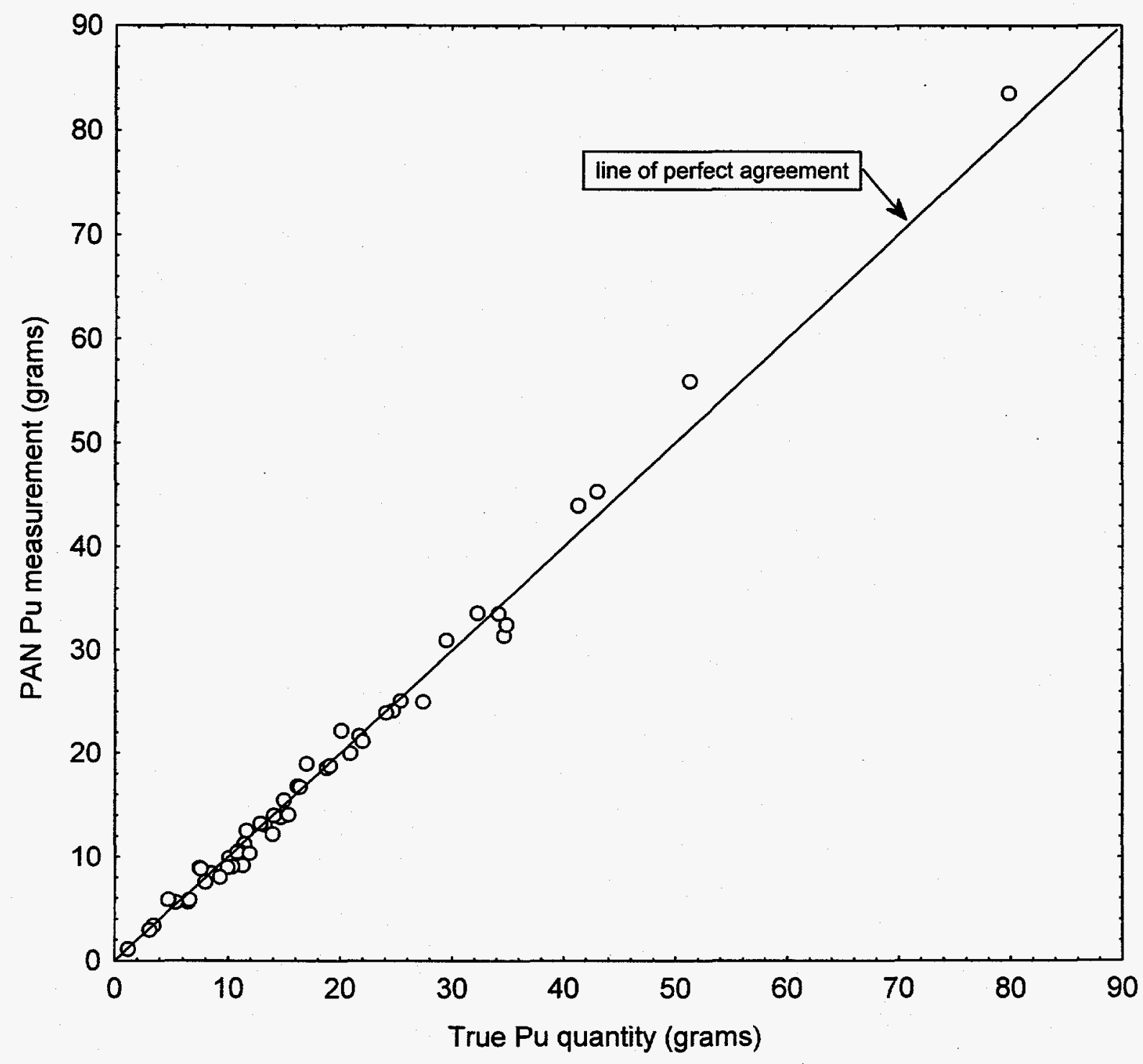


Figure 9-2. Bias in the PAN system measurements for the simulated graphite waste drums (infinite count time data)

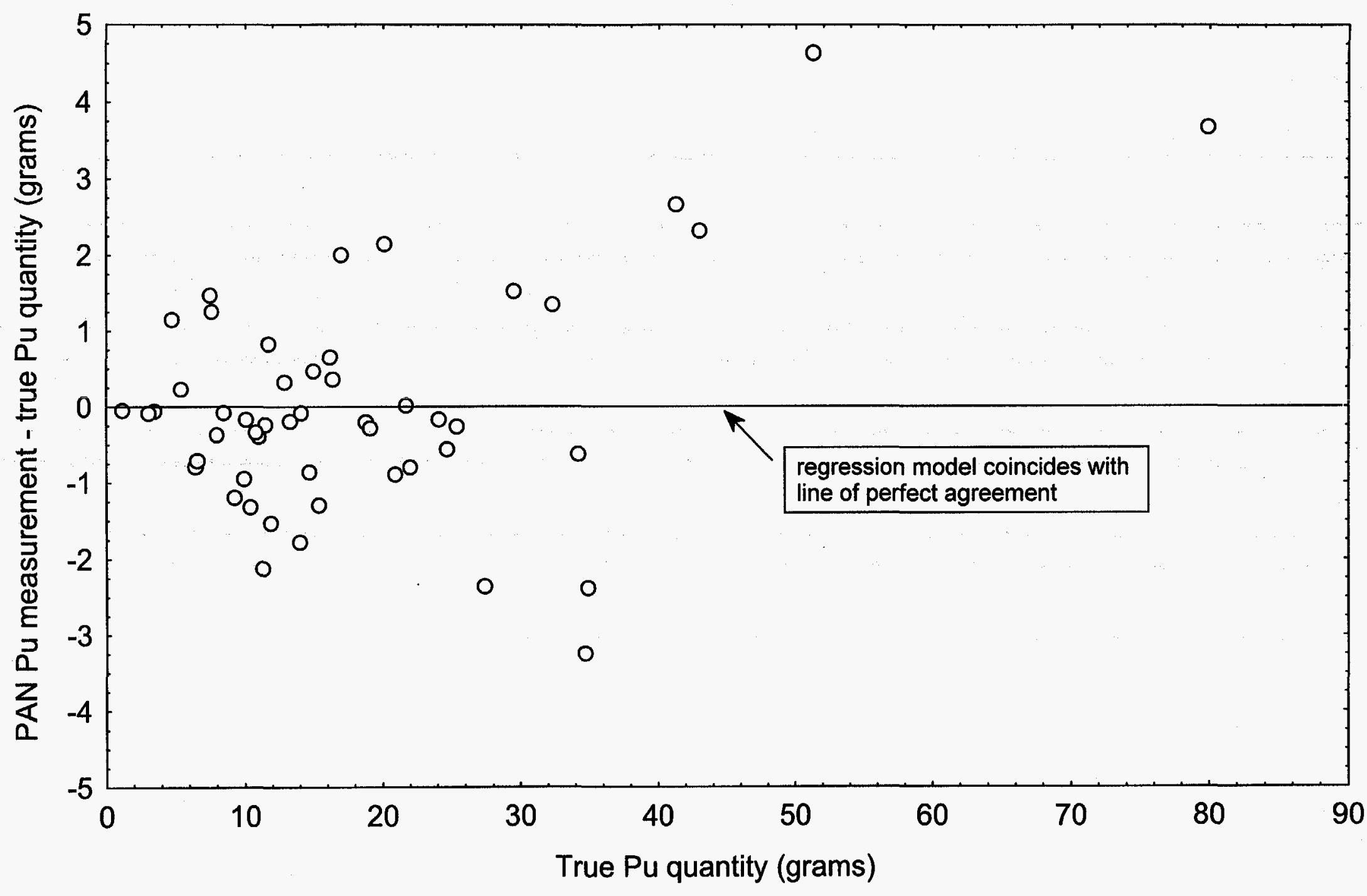




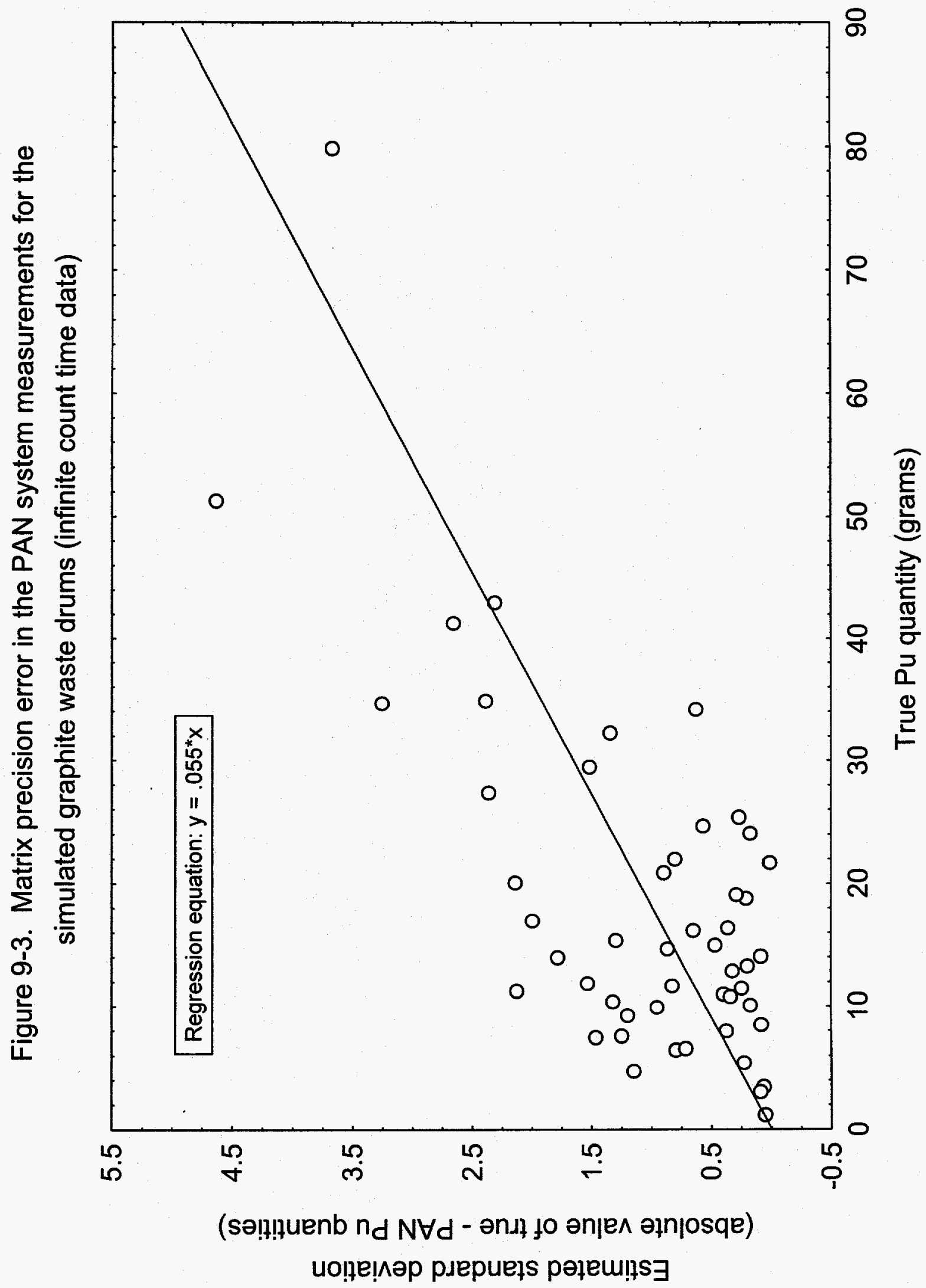




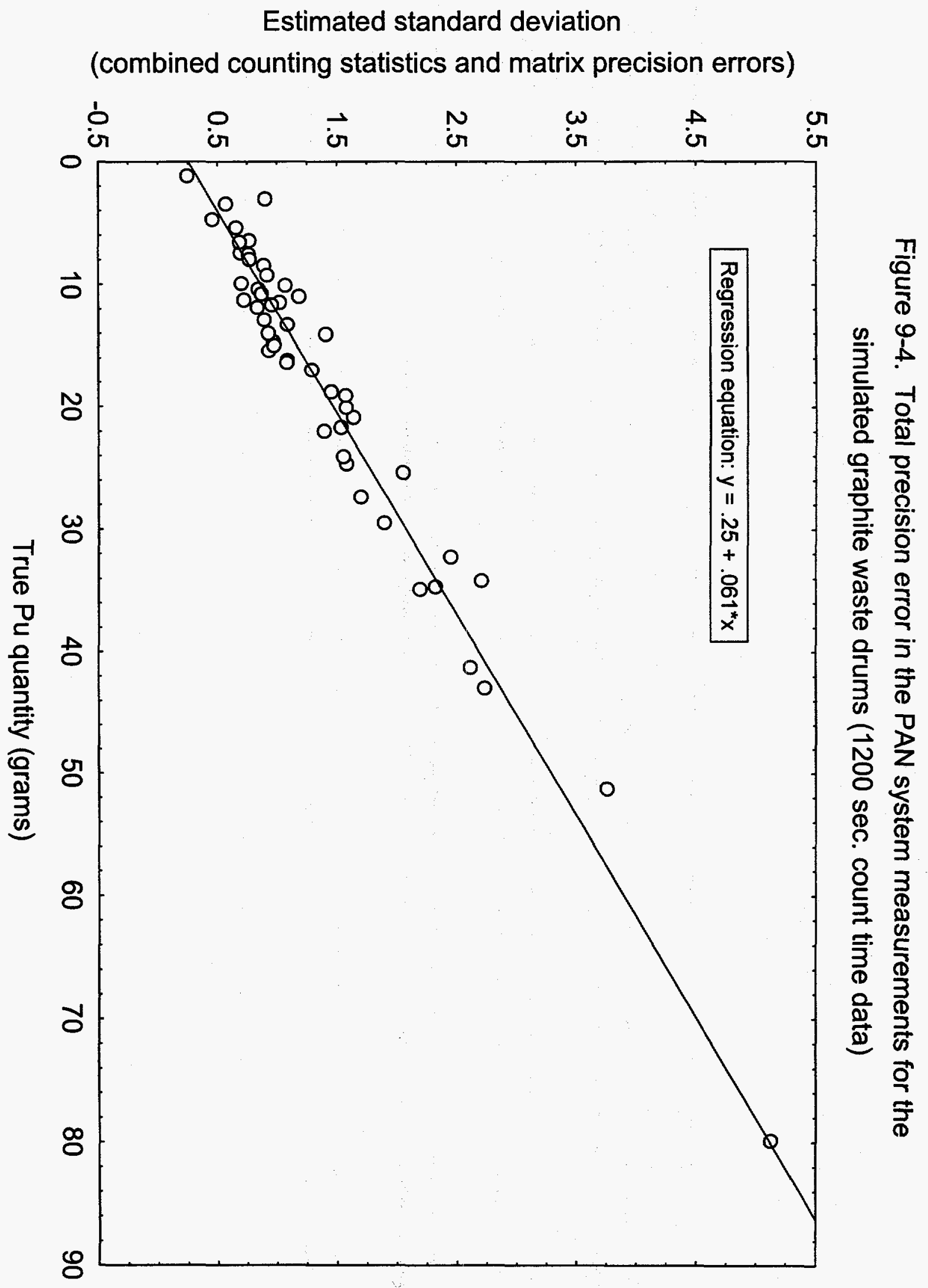




\section{SUMMARY AND DISCUSSION OF RESULTS}

This report covers a methodology for determining the total uncertainty of the PAN drum assay system and applies this methodology to the graphite content code 300 . In order to perform this evaluation, a complete assessment of the physical and radiological characteristics of graphite content code 300 waste drums was performed. A multivariate statistical model was developed which accounted for variations and correlations of parameters which could alter the response of the assay system. The primary parameters in this model are: mass of WG PU, mass fraction of Pu-240, fill height of the drum, average matrix density, absorber index, background singles counts, background coincidence counts, and fraction of total $\mathrm{Pu}$ contained in $\mathrm{Pu}$ chunks. Real time radiography tapes, data from past measurements using the PAN assay system and shipping records from Rocky Flats Plant were the sources of empirical data used in this assessment. From this study a set of 50 cases simulating the waste characteristics for graphite were prepared for analysis using a computer simulation of the assay system response and analysis routines.

The computer simulation of the PAN assay system response involved a neutron transport calculation using MCNP and a follow-up program which uses the MCNP results along with other parameters in the statistical model to determine the singles and coincidence count rate responses. These count rate data were then analyzed to yield Pu mass using exactly the same algorithms as contained in the PAN analysis program. The accuracy of the combined simulation routine was verified using data taken from measurements involving a surrogate graphite waste drum and disk Pu sources. From these comparisons small adjustment factors were derived to make the simulated Pu mass quantities agree on average with the Pu mass determined from the PAN measurement. These adjustment factors were included in the computer simulation routine in all analyses involving the Latin hypercube cases so that there was no bias in the simulation results relative to the expected PAN results.

Analysis of the results for the simulated waste drums indicates that, on average, there is no bias in the PAN system measurements of graphite waste. The matrix precision error, i.e., the precision error due to variability in the waste matrix from drum to drum, is estimated to be $5.5 \%$ of the true Pu quantity in the drum.

Other follow-on calculations, primarily for producing certification reports for multiple drum units, are made in other documents and software related to the PAN system. These follow-on calculations make use of the results derived in this report. A table listing the various parameters required for those followon calculations is given in Appendix B.

\subsection{Comparison of Results to QAPP QAOs for Radioassay Systems}

Using the results of this evaluation we can make comparisons with quality assurance objectives (QAOs) as established in Table 9-1 of the TRU Waste Characterization Quality Assurance Program Plan (U. S. DOE, 1996). The parameter of interest in this table is "Total Bias" which is what has been evaluated in this report. QAOs are given for four different levels of total alpha activity or the equivalent quantity of weapons grade Pu. Equation 9.4 establishes the uncertainty in the total bias in the PAN Pu measurements for graphite waste drums for any quantity of $\mathrm{Pu}$. Approximate $95 \%$ confidence interval bounds for the true Pu based on Equation 9.4 and the finding that the estimated bias in the PAN system for graphite is zero are

$0 \pm 2 s_{b_{x}}$. 
This equation reduces to $0 \pm 2 \%$ of the actual Pu mass being measured.

While Equations 9.4 and 10.1 express bias as the difference between measured and true values, the QAO expresses it as the ratio of the measured to true values. That is, if $b$ is the bias in the true measurement of $\mathrm{x}$ grams Pu as estimated in this report, then the QAO bias measure is $100 *(x+b) / x$. Table $10-1$ gives the bias limits for the four different Pu mass levels specified in the QAPP and compares them to the QAPP QAO limits.

Table 10-1. Comparison of QAPP QAO limits for bias to calculated $95 \%$ bounds for PAN bias.

\begin{tabular}{|c|c|c|c|c|}
\hline $\begin{array}{c}\text { Nominal } \\
\begin{array}{c}\text { Point } \\
(\mathrm{g} \mathrm{Pu})\end{array}\end{array}$ & $\begin{array}{c}\text { Allowable } \\
\text { lower bound } \\
\text { for total bias } \\
(\%)\end{array}$ & $\begin{array}{c}\text { Allowable } \\
\text { upper bound } \\
\text { for total bias } \\
(\%)\end{array}$ & $\begin{array}{c}\text { PAN lower } \\
\text { bound for total } \\
\text { bias } \\
(\%)\end{array}$ & $\begin{array}{c}\text { PAN upper } \\
\text { bound for total } \\
\text { bias } \\
(\%)\end{array}$ \\
\hline .1 & 25 & 400 & 98 & 102 \\
\hline 1.0 & 35 & 300 & 98 & 102 \\
\hline 10 & 67 & 150 & 98 & 102 \\
\hline 160 & 67 & 150 & 98 & 102 \\
\hline
\end{tabular}

Since the standard deviation $\mathrm{s}_{\mathrm{b}_{\mathrm{x}}}$ is proportional to $\mathrm{x}$, the quantity of $\mathrm{Pu}$, the relative bounds are the same for all four compliance point levels. The relative bias error bounds of $0 \pm 2 \%$ for the PAN system easily meet the QAPP requirements.

In order to be critically safe, there is a safety limit requiring that the measured Pu mass plus 2 times its standard error must not exceed $200 \mathrm{~g}$. Based on the equations derived in this report the measured mass must be below approximately $177 \mathrm{~g}$ in order to meet this criterion for drums containing graphite waste. 


\section{REFERENCES}

Becker, G. K. (1993). "Drum Neutron Counter Chamber and Detector Configuration," Idaho National Engineering Laboratory Engineering Design File Document EDF-RWMC-606.

Becker, G. K. (1995). "SWEPP Assay System Graphite Calibration Drum Design and Material Specification," Idaho National Engineering Laboratory Engineering Design File Document EDF-RWMC672.

Briesmeiser, J. F. (1986). "MCNP - A General Purpose Monte Carlo Code for Neutron and Photon Transport, Version 3A," Los Alamos National Laboratory manual LA-7396-M Rev 2.

East, L. V., Marwil, E. S., and Matthews, S. D. (1997). "SWEPP Assay System Version 2.1 Software Requirements Specification," Idaho National Engineering and Environmental Laboratory Report INEL/EXT-97-00363.

Harker, Y. D., (1997). "Zero Matrix Calibration of the SWEPP PAN System," Idaho National Engineering and Environmental Laboratory Report INEEL/INT-97-00866.

Harker, Y. D., Blackwood, L. G., Meachum, T. R. (1995). "Uncertainty Analysis of the SWEPP Drum Assay System for Graphite Content Code 300. Idaho National Engineering Laboratory Report INEL95/475.

Hogg, R. V. and Craig, A. T. (1978). Introduction to Mathematical Statistics, 4th Edition, New York: Macmillan Publishing.

Iman, R. L. and Conover, W. J. (1982). "A Distribution-Free Approach to Inducing Rank Correlation Among Input Variables," Communications in Statistics, B11(3), 311-334.

Iman, R. L. and Helton, J. C. (1985). "A Comparison of Uncertainty and Sensitivity Analysis Techniques for Computer Models." Technical Report SAND84-1461, Sandia Laboratories, Albuquerque, New Mexico.

Iman, R. L. and Shortencarier, M. J. (1984). "A FORTRAN 77 Program and User's Guide for the Generation of Latin Hypercube and Random Samples for Use With Computer Models." NUREG/CR-3624. Sandia National Laboratories.

Madansky, A. (1988). Prescriptions for Working Statisticians, New York: Springer-Verlag.

Meachum, T. R. (1997). "QA of SWEPP Assay System Simulation Application,” Idaho National Engineering and Environmental Laboratory Engineering Design File INEL-95/246 (Rev 2).

Neter, J. and Wasserman, W. (1974). Applied Linear Statistical Models: Regression, Analysis of Variance, and Experimental Designs, Homewood, IL: Richard D. Irwin, Inc.

Reilly, D., Ensslin, N., Smithe, Jr., H., and Kreiner, S. (1991). Passive Nondestructive Assay of Nuclear Materials, Washington, D. C., U. S. Nuclear Regulatory Commission. 
U. S. Department of Energy (1996). TRU Waste Characterization Quality Assurance Program Plan, Interim Change. CAO-94-1010.

Yoon, W. Y. (1993). "MCNP Modeling and Verification Calculations I," Idaho National Engineering Laboratory Engineering Design File Document EDF-RWMC-609. 


\section{APPENDIX A SPATIAL VARIATION OF DENSITY AND PU MASS FRACTION}

In modeling the spatial dependence of density and Pu mass fraction, one assumes that both are distributed in the drum according to an equal probability weighting: i.e., the probability per unit volume is independent of the position within the matrix in the drum. On average this amounts to a uniform distribution; however for any particular drum there can be a variation in either density or Pu mass fraction based on the statistical distribution assigned to the respective variable. The objective of this appendix is to arrive at a method for estimating the statistical variance of either the density or Pu mass fraction distributions.

For the inventory of drums at SWEPP, there is no quantitative data available on spatial variation of density or Pu fines mass fraction in a drum. In lieu of direct spatial data, one must use drum-to-drum variations of the average density in a drum or the average $\mathrm{Pu}$ mass in a drum and assume that the drumto-drum variations are directly linked to the corresponding spatial variations within a drum.

\section{Spatial Variation of Density}

To address the link between the drum-to-drum variation in average density and the spatial distribution of density within a drum, let's consider that a drum is subdivided into $\mathrm{N}$ zones and assume that the density variation from drum-to-drum results from zone-to-zone variations of the zonal density. The average density, $\mathrm{D}$, for a drum is the sum of the individual zone masses divided by the total matrix volume.

$$
D=\frac{\sum_{i=1}^{N} M_{i}}{\sum_{j=1}^{N} V_{j}}=\frac{\sum_{i=1}^{N} M_{i}}{N V_{o}}=\frac{\sum_{i=1}^{N} D_{i}}{N}
$$

where:

$$
\begin{aligned}
& M_{i}=\text { the mass of zone } i, \\
& V_{i}=\text { the volume of zone } i, \\
& D_{i}=\text { the density of zone } i .
\end{aligned}
$$

The second and third expressions in Equation (A.1) are based on all zones having the same volume, $\mathrm{V}_{\mathrm{o}}$.

Using the standard formula for propagation of errors in quadrature, the standard deviation of the average density, $\sigma_{\mathrm{D}}$, can be evaluated in terms of the standard deviations of the individual zone densities, $\sigma_{\mathrm{Di}}$.

$$
\sigma_{D}=\frac{1}{N} \sqrt{\sum_{i=1}^{N} \sigma_{D i}^{2}}
$$

Now let's assume that the zone densities follow the same statistical distribution independent of position and have the same standard deviation for each zone, $\sigma_{D o}$. This assumption is just a restatement of the basic equal probability assumption. With this simplification, Equation (A.2) reduces to: 
$\sigma_{\mathrm{D}}=\frac{\sigma_{\mathrm{D}}}{\sqrt{\mathrm{N}}}$

Thus, there is a simple relationship between the standard deviation for the average density in a drum and the standard deviation for zone densities within a drum. This relationship allows one to use the statistical distribution of drum density as determined from statistical analysis of net weights and matrix volumes for drums of a particular content code in the SWEPP inventory and estimate the zone matrix density distribution within a drum. Equation (A.3) does show that variations in zone density increase by the square root of the number of zones into which the drum is being subdivided. For the drum model reported here there are 24 zones in the volume assigned to waste matrix and therefore the variation in zone density can be expected to be a factor of 5 larger than the variation of drum average density.

\section{Spatial Variation of Plutonium Density}

Using the same arguments developed above one can come up with a similar relationship between zonal $\mathrm{Pu}$ density standard deviations and drum Pu density standard deviation

$\sigma_{\mathrm{P}}=\frac{\sigma_{\mathrm{Po}}}{\sqrt{\mathrm{N}}}$

where:

$$
\begin{aligned}
& \sigma_{\mathrm{P}}=\text { is the standard deviation of the drum Pu density and } \\
& \sigma_{\mathrm{Po}}=\text { the standard deviation of the zonal Pu density which as above is taken to be the same for } \\
& \text { each zone. }
\end{aligned}
$$

Since the Pu mass fraction is used directly in specifying the input to the MCNP model, it is instructive to determine the variation of this variable as a function of zone. The zonal $\mathrm{Pu}$ mass fraction, $\mathrm{MF}_{\mathrm{i}}$, is equal to the zonal $\mathrm{Pu}$ density, $\mathrm{P}_{\mathrm{i}}$, divided by the zonal matrix density, $\mathrm{D}_{\mathrm{i}}$. The relative standard deviation for the zonal Pu mass fraction can be shown to be:

$$
\frac{\sigma_{\mathrm{MFi}}}{\mathrm{MF}_{\mathrm{i}}}=\frac{1}{\sqrt{\mathrm{N}}} \sqrt{\left(\frac{\sigma_{\mathrm{P}}}{\mathrm{P}_{\mathrm{i}}}\right)^{2}+\left(\frac{\sigma_{\mathrm{D}}}{\mathrm{D}_{\mathrm{i}}}\right)^{2}}
$$

Now if one assumes that the $D_{i} s$ and $P_{i} s$ do not vary significantly over the zones then they can be replaced by the values of $M$ and $P$ for the drum and Equation (A.5) reduces to:

$$
\frac{\sigma_{\mathrm{MFo}}}{\mathrm{MF}_{\mathrm{o}}}=\frac{1}{\sqrt{\mathrm{N}}} \sqrt{\left(\frac{\sigma_{\mathrm{P}}}{\mathrm{P}}\right)^{2}+\left(\frac{\sigma_{\mathrm{D}}}{\mathrm{D}}\right)^{2}}
$$

The square root on the right side of Equation A.6 is equal to the relative standard deviation for the Pu mass fraction for the drum, $\sigma_{\mathrm{MF}} \mathrm{MF}$. Therefore, Equation $\mathrm{A} .6$ is reduced to a similar relationship as found for matrix density and plutonium density. 


\section{APPENDIX B \\ TABLE OF PARAMETERS REQUIRED FOR FOLLOW-ON CALCULATIONS}

The table below lists parameter values and associated errors generated in this report that are used in subsequent calculations in the processing of PAN data. The subsequent calculations performed are documented in the INEEL engineering design file (internal publication INEL/INT-97-00140) titled "Use of SWEPP Assay System Version 2.1 Analysis Results to Produce Multiple Drum Certification Reports." Parameter names listed are those used in the engineering design file.

\begin{tabular}{|l|l|c|c|}
\hline \multicolumn{1}{|c|}{ Parameter Name } & \multicolumn{1}{|c|}{ Description } & Value & Error \\
\hline $\mathrm{PB}_{\mathrm{m}}$ & $\begin{array}{l}\text { Passive mass bias correction } \\
\text { coefficient (intercept) }\end{array}$ & 0 & 0 \\
\hline $\mathrm{PB} 1_{\mathrm{m}}$ & $\begin{array}{l}\text { Passive mass bias correction } \\
\text { coefficient (slope) }\end{array}$ & 1 & 0 \\
\hline Cov(PB0, PB1) & Covariance of $\mathrm{PB} 0_{\mathrm{m}}$ and $\mathrm{PB} 1_{\mathrm{m}}$ & 0 & $-^{*}$ \\
\hline PA0 & $\begin{array}{l}\text { Passive matrix precision coefficient } \\
\text { (intercept) }\end{array}$ & 0 & 0 \\
\hline PA1 & $\begin{array}{l}\text { Passive matrix precision coefficient } \\
\text { (slope) }\end{array}$ & .055 & --* $^{*}$ \\
\hline PA2 & $\begin{array}{l}\text { Passive matrix precision coefficient } \\
\text { (quadratic) }\end{array}$ & 0 & 0 \\
\hline
\end{tabular}

*not required 